\title{
An Improved and Flexible Synthetic Access to the Spiroindole Backbone of Cebranopadol
}

Daniel Wachtendorf, Marc Schmidtmann, and Jens Christoffers*

Institut für Chemie, Universität Oldenburg, Carl-von-Ossietzky-Str. 9-11, D-26111 Oldenburg,

Germany

Supporting Information

1. General Information $\quad S 2$

2. Procedures and spectroscopic data of all compounds $\quad$ S2

3. Preparation of compound $\mathbf{5}$ - optimization $\quad \mathrm{S} 18$

4. Crystal structure of compound cis-11a $\quad$ S20

5. References $\quad$ S23

6. ${ }^{1} \mathrm{H},{ }^{13} \mathrm{C}\left\{{ }^{1} \mathrm{H}\right\}$ and ${ }^{19} \mathrm{~F}\left\{{ }^{1} \mathrm{H}\right\}$ NMR spectra $\quad \mathrm{S} 24$ 


\section{General Information}

Heating of reaction mixtures was always performed with an oil bath. Preparative co-

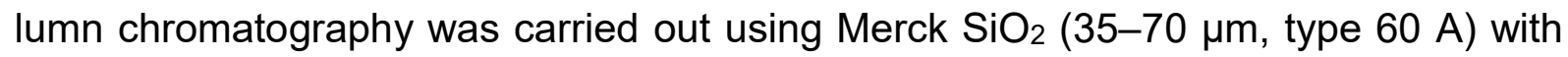
hexanes (mixture of isomers, bp. $64-71^{\circ} \mathrm{C}$ ) and tert-butyl methyl ether (MTBE) as eluents. TLC was performed on aluminum plates coated with $\mathrm{SiO}_{2} \mathrm{~F}_{254} \cdot{ }^{1} \mathrm{H},{ }^{19} \mathrm{~F}$ and ${ }^{13} \mathrm{C}$ NMR spectra were recorded on Bruker Avance DRX 500 and 300 instruments. Multiplicities of carbon signals were determined with DEPT experiments. MS and HRMS spectra of products were obtained with a Waters Q-TOF Premier (ESI, positive mode), Micromass Q-TOF micro (ESI, negative mode) or Thermo Scientific DFS (EI) spectrometers. IR spectra were recorded on a Shimadzu IR Spirit T spectrometer equipped with a diamond ATR unit. Compounds $6,{ }^{\mathrm{S1}} 10 \mathrm{a},{ }^{\mathrm{S} 2}$ 10b,,${ }^{\mathrm{S} 3}$ 10c,${ }^{\mathrm{S4}} 10 \mathrm{~d}^{\mathrm{S3}}$ and N-Boc-L$\mathrm{NpgOH}^{55}$ were literature known and prepared accordingly. All other starting materials were commercially available.

\section{Procedures and spectroscopic data of all compounds}

(Nitromethyl)benzene (6). ${ }^{\mathrm{S} 1} \mathrm{H}_{2} \mathrm{O}_{2}\left(30 \%\right.$ in $\left.\mathrm{H}_{2} \mathrm{O}, 106 \mathrm{~g}, 935 \mathrm{mmol}\right)$ was slowly added to a cooled (ice-water bath) solution of trifluoracetic anhydride $(140 \mathrm{~g}, 668 \mathrm{mmol})$ in MTBE $(100 \mathrm{~mL})$. After further stirring for $15 \mathrm{~min}$, the mixture was poured to a suspension of benzaldoxime $7(16.3 \mathrm{~g}, 134 \mathrm{mmol})$, urea $(4.00 \mathrm{~g}, 66.8 \mathrm{mmol})$ and $\mathrm{Na}_{2} \mathrm{HPO}_{4}$. $2 \mathrm{H}_{2} \mathrm{O}(136 \mathrm{~g}, 761 \mathrm{mmol})$ in MTBE $(250 \mathrm{~mL})$. The resulting mixture was stirred at $55^{\circ} \mathrm{C}$ for $16 \mathrm{~h}$. After cooling to ambient temperature, the mixture was filtered, diluted with saturated, aqueous $\mathrm{NH}_{4} \mathrm{Cl}$ solution $(100 \mathrm{~mL})$ and extracted with MTBE $(3 \times 200 \mathrm{~mL})$. The combined organic layers were dried $\left(\mathrm{MgSO}_{4}\right)$, filtered and the solvent was evaporated in vacuo to furnish the title compound $6(17.5 \mathrm{~g}, 128 \mathrm{mmol}, 96 \%)$ as a yellow oil, which did not require further purification. ${ }^{1} \mathrm{H} \mathrm{NMR}\left(300 \mathrm{MHz}, \mathrm{CDCl}_{3}\right): \delta=5.44(\mathrm{~s}, 2 \mathrm{H})$, $7.40-7.50(\mathrm{~m}, 5 \mathrm{H}) \mathrm{ppm}$. All spectroscopic data are in accordance with the literature. ${ }^{\mathrm{S} 6}$ $\mathrm{C}_{7} \mathrm{H}_{7} \mathrm{NO}_{2}\left(137.14 \mathrm{~g} \mathrm{~mol}^{-1}\right)$.

Dimethyl 4-Nitro-4-phenylpimelate (8). A solution of DBU $(9.97 \mathrm{~g}, 65.5 \mathrm{mmol})$ in $\mathrm{CH}_{2} \mathrm{Cl}_{2}(25 \mathrm{~mL}$ ) was added slowly (within $10 \mathrm{~min}$ ) at ambient temperature to a solution of compound $6(18.0 \mathrm{~g}, 131 \mathrm{mmol})$ and methyl acrylate $(25.9 \mathrm{~g}, 301 \mathrm{mmol})$ in $\mathrm{CH}_{2} \mathrm{Cl}_{2}$ $\left(25 \mathrm{~mL}\right.$ ). After stirring for $16 \mathrm{~h}$ at $50^{\circ} \mathrm{C}$ and subsequent cooling to ambient temperature, the mixture was diluted with brine $(100 \mathrm{~mL})$ and extracted with $\mathrm{CH}_{2} \mathrm{Cl}_{2}(3 \times 60 \mathrm{~mL})$. The combined organic layers were dried $\left(\mathrm{MgSO}_{4}\right)$, filtered and the solvent was evaporated 
in vacuo. The residue was heated to reflux ( $5 \mathrm{~min}$ ) with hexane $(100 \mathrm{~mL})$. After cooling to ambient temperature, the upper hexane layer was separated and the lower, crude product containing layer was again extracted with hexane $\left(100 \mathrm{~mL}, 5 \mathrm{~min}\right.$ at $\left.100^{\circ} \mathrm{C}\right)$. After repeating this extraction all together four times, the lower layer did not contain any product 8 , as monitored by ${ }^{1} \mathrm{H}$ NMR. The combined hexane layers were evaporated to yield compound $8(35.9 \mathrm{~g}, 116 \mathrm{mmol}, 89 \%)$ as a colorless solid, mp. $43-45^{\circ} \mathrm{C}$. Alternatively, particularly for smaller scales, the product 8 could be obtained by column chromatography $\left(\mathrm{SiO}_{2}\right.$, hexanes/MTBE 1:1, $\left.\mathrm{Rf}_{\mathrm{f}}=0.34\right) .{ }^{1} \mathrm{H} \mathrm{NMR}\left(500 \mathrm{MHz}, \mathrm{CDCl}_{3}\right): \delta$ $=2.17-2.32(\mathrm{~m}, 4 \mathrm{H}), 2.66-2.76(\mathrm{~m}, 4 \mathrm{H}), 3.65(\mathrm{~s}, 6 \mathrm{H}), 7.29-7.34(\mathrm{~m}, 2 \mathrm{H}), 7.38-7.47$ (m, 3H) ppm. ${ }^{13} \mathrm{C}\left\{{ }^{1} \mathrm{H}\right\} \mathrm{NMR}\left(125 \mathrm{MHz}, \mathrm{CDCl}_{3}\right): \delta=29.1\left(2 \mathrm{CH}_{2}\right), 31.5\left(2 \mathrm{CH}_{2}\right), 52.1(2$ $\left.\mathrm{CH}_{3}\right), 95.5(\mathrm{C}), 125.7(2 \mathrm{CH}), 129.2(2 \mathrm{CH}), 129.3(\mathrm{CH}), 137.4(\mathrm{C}), 172.5(2 \mathrm{C}) \mathrm{ppm}$. IR (ATR): 2952 (w), 1732 (s), 1539 (s), 1502 (w), 1459 (w), $1449(\mathrm{~m}), 1432(\mathrm{~m}), 1413$ (w), $1384(w), 1350(m), 1330(w), 1300(m), 1193(s), 1167(s), 1077(w), 1060(w)$, $1020(w), 983(w), 894(w), 827(w), 800(w), 779(w), 763(w), 726(m), 696(s), 649$ (w), $583(\mathrm{~m}) \mathrm{cm}^{-1}$. HRMS (ESI, pos. mode) $\mathrm{m} / \mathrm{z}:[\mathrm{M}+\mathrm{Na}]^{+}$calcd. for $\mathrm{C}_{15} \mathrm{H}_{19} \mathrm{NNaO}_{6}{ }^{+}$ 332.1110; found 332.1102. $\mathrm{C}_{15} \mathrm{H}_{19} \mathrm{NO}_{6}\left(309.32 \mathrm{~g} \mathrm{~mol}^{-1}\right)$.

Methyl 5-Nitro-5-phenyl-2-cyclohexanone-1-carboxylate (9). A solution of pimelate 8 (15.3 g, $49.5 \mathrm{mmol})$ and $\mathrm{MeOH}(0.16 \mathrm{~g}, 5.0 \mathrm{mmol})$ in THF $(40 \mathrm{~mL})$ was slowly poured into a suspension of $\mathrm{NaH}(3.35 \mathrm{~g}, 60 \%$ dispersion in mineral oil, $83.8 \mathrm{mmol})$ in THF $(40 \mathrm{~mL})$. The mixture was stirred $2 \mathrm{~h}$ at ambient temperature and further $1 \mathrm{~h}$ at $50^{\circ} \mathrm{C}$. After cooling to ambient temperature, the solution was diluted with saturated, aqueous citric acid solution $(80 \mathrm{~mL})$ and extracted with MTBE $(3 \times 100 \mathrm{~mL})$. The combined organic layers were dried $\left(\mathrm{MgSO}_{4}\right)$, filtered and the solvent was evaporated in vacuo. After addition of $\mathrm{MeOH}(10 \mathrm{~mL})$ the residue crystallized. It was washed with $\mathrm{MeOH}$ $(100 \mathrm{~mL})$ and dried in high vacuum. Compound $9(7.41 \mathrm{~g}, 26.7 \mathrm{mmol}, 54 \%)$ was obtained as a colorless solid, mp. $119-121^{\circ} \mathrm{C}$. In the NMR spectra, exclusively the enol tautomer is detected. ${ }^{1} \mathrm{H}$ NMR $\left(500 \mathrm{MHz}, \mathrm{CDCl}_{3}\right): \delta=2.28-2.38(\mathrm{~m}, 1 \mathrm{H}), 2.46-2.55$ (m, 2H), 2.89-2.95 (m, 1H), $3.06(\mathrm{~d}, J=16.8 \mathrm{~Hz}, 1 \mathrm{H}), 3.59(\mathrm{~d}, J=16.6 \mathrm{~Hz}, 1 \mathrm{H}), 3.82$ (s, 3H), 7.36-7.42 (m, 3H), 7.45-7.49 (m, 2H), $12.12(\mathrm{~s}, 1 \mathrm{H}) \mathrm{ppm} .{ }^{13} \mathrm{C}\left\{{ }^{1} \mathrm{H}\right\} \mathrm{NMR}(125$ $\left.\mathrm{MHz}, \mathrm{CDCl}_{3}\right): \delta=26.3\left(\mathrm{CH}_{2}\right), 30.1\left(\mathrm{CH}_{2}\right), 31.9\left(\mathrm{CH}_{2}\right), 51.9\left(\mathrm{CH}_{3}\right), 90.4(\mathrm{C}), 94.6(\mathrm{C})$, $125.3(2 \mathrm{CH}), 129.1(2 \mathrm{CH}), 129.5(\mathrm{CH}), 137.8(\mathrm{C}), 170.3(\mathrm{C}), 171.8(\mathrm{C})$ ppm. IR (ATR): $2952(\mathrm{w}), 1674(\mathrm{~s}), 1626(\mathrm{~m}), 1534(\mathrm{~s}), 1502(\mathrm{w}), 1433(\mathrm{~s}), 1342(\mathrm{~m}), 1296(\mathrm{~m}), 1267$ $(m), 1227(m), 1216(m), 1197(m), 1189(m), 1154(w), 1139(w), 1077(m), 1054(w)$, 
$1039(\mathrm{w}), 1010(\mathrm{~m}), 949(\mathrm{w}), 870(\mathrm{w}), 813(\mathrm{~m}), 773(\mathrm{~s}), 764(\mathrm{~s}), 706(\mathrm{~m}), 691(\mathrm{~m}), 624$ (m), $593(\mathrm{~m}) \mathrm{cm}^{-1} . \mathrm{MS}(\mathrm{El}, 70 \mathrm{eV}): \mathrm{m} / \mathrm{z}(\%) 277$ (3) [M] ${ }^{+}, 230$ (40), 199 (70), 171 (19), 157 (100), 141 (14), 128 (26), 115 (32), 91 (43), 77 (12), 55 (40). HRMS (El, $70 \mathrm{eV}$ ) $\mathrm{m} / \mathrm{z}$ : $[\mathrm{M}]^{+}$calcd. for $\mathrm{C}_{14} \mathrm{H}_{15} \mathrm{NO}_{5}{ }^{+} 277.0945$; found 277.0952 . $\mathrm{C}_{14} \mathrm{H}_{15} \mathrm{NO}_{5}(277.28 \mathrm{~g}$ $\left.\mathrm{mol}^{-1}\right)$.

4-Nitro-4-phenylcyclohexanone (5). A solution of ester 9 ( $2.0 \mathrm{~g}, 7.2 \mathrm{mmol}), \mathrm{LiBr}$ (1.88 $\mathrm{g}, 21.6 \mathrm{mmol})$ and camphor sulfonic acid $(3.35 \mathrm{~g}, 14.4 \mathrm{mmol})$ in DMPU $(48 \mathrm{~mL}$ was stirred under nitrogen atmosphere for $16 \mathrm{~h}$ at $100^{\circ} \mathrm{C}$. After cooling to ambient temperature, the solution was diluted with EtOAc $(250 \mathrm{~mL})$ and washed with brine $(3 \times 200$ $\mathrm{mL})$. The organic layers were dried $\left(\mathrm{MgSO}_{4}\right)$, filtered and the solvent was evaporated in vacuo. The residue was chromatographed $\left(\mathrm{SiO}_{2}\right.$, hexanes/MTBE $\left.2: 1, \mathrm{R}_{\mathrm{f}}=0.21\right)$ to furnish cyclohexanone 5 (961 mg, $4.38 \mathrm{mmol}, 61 \%)$ as a colorless solid, mp. 128$130^{\circ} \mathrm{C} .{ }^{1} \mathrm{H}$ NMR $\left(500 \mathrm{MHz}, \mathrm{CDCl}_{3}\right): \delta=2.45-2.62(\mathrm{~m}, 6 \mathrm{H}), 3.18-3.28(\mathrm{~m}, 2 \mathrm{H}), 7.39$ $7.47(\mathrm{~m}, 3 \mathrm{H}), 7.49-7.54(\mathrm{~m}, 2 \mathrm{H}) \mathrm{ppm} .{ }^{13} \mathrm{C}\left\{{ }^{1} \mathrm{H}\right\} \mathrm{NMR}\left(125 \mathrm{MHz}, \mathrm{CDCl}_{3}\right): \delta=34.4(2$ $\left.\mathrm{CH}_{2}\right), 37.4\left(2 \mathrm{CH}_{2}\right), 91.1(\mathrm{C}), 125.3(2 \mathrm{CH}), 129.4(2 \mathrm{CH}), 129.8(\mathrm{CH}), 137.4(\mathrm{C}), 207.6$ (C) ppm. IR (ATR): 2976 (w), 2927 (w), 1706 (s), 1537 (s), 1502 (m), 1449 (m), 1430 $(\mathrm{m}), 1370(\mathrm{w}), 1346(\mathrm{~m}), 1269(\mathrm{w}), 1254(\mathrm{w}), 1223(\mathrm{~m}), 1139(\mathrm{~m}), 1117(\mathrm{~m}), 1077(\mathrm{w})$, $1004(\mathrm{w}), 984(\mathrm{w}), 962(\mathrm{~m}), 840(\mathrm{w}), 819(\mathrm{~s}), 764(\mathrm{~s}), 744(\mathrm{~m}), 714(\mathrm{~m}), 696(\mathrm{~s}), 611$ (w), $560(\mathrm{~m}) \mathrm{cm}^{-1}$. HRMS (ESI, pos. mode) $\mathrm{m} / \mathrm{z}$ : [M $\left.+\mathrm{Na}\right]^{+}$calcd. for $\mathrm{C}_{12} \mathrm{H}_{13} \mathrm{NNaO}_{3}{ }^{+}$ 242.0793; found 242.0796. $\mathrm{C}_{12} \mathrm{H}_{13} \mathrm{NO}_{3}\left(219.24 \mathrm{~g} \mathrm{~mol}^{-1}\right)$.

\section{General Procedure A for the Preparation of 3-(2-Hydroxyethyl)indole Derivatives} 10a-10d (GP A): Following a literature report, ${ }^{\mathrm{S} 7}$ 2,3-dihydrofurane (1.1 eq.) was added slowly at $100^{\circ} \mathrm{C}$ to a stirred solution of the respective arylhydrazine hydrochloride (1.0 eq.) in $\mathrm{DMA} / \mathrm{H}_{2} \mathrm{SO}_{4}\left(4 \%\right.$ aqueous) $(1: 1,3 \mathrm{~L} / \mathrm{mol})$. After stirring for $90 \mathrm{~min}$ at $100^{\circ} \mathrm{C}$, the solution was cooled to ambient temperature, diluted with EtOAc $(3 \mathrm{~L} / \mathrm{mol})$ and washed with brine $(2 \mathrm{~L} / \mathrm{mol})$. The organic layer was dried $\left(\mathrm{MgSO}_{4}\right)$, filtered and the solvent was evaporated in vacuo. The residue was chromatographed $\left(\mathrm{SiO}_{2}\right.$, hexanes/MTBE) to furnish indole derivatives 10a-10d. 
3-(2-Hydroxyethyl)indole (10a). ${ }^{\text {S2 }}$ According to the GP A, phenylhydrazine hydrochloride $(2.00 \mathrm{~g}, 13.8 \mathrm{mmol})$ and 2,3-dihydrofurane $(1.07 \mathrm{~g}, 15.2 \mathrm{mmol})$ were converted in DMA $/ \mathrm{H}_{2} \mathrm{SO}_{4} / \mathrm{H}_{2} \mathrm{O}(40 \mathrm{~mL})$ at $100^{\circ} \mathrm{C}$ for 90 min to furnish the product $10 \mathrm{a}$ (890 mg, $5.50 \mathrm{mmol}, 40 \%)$ after chromatography $\left(\mathrm{SiO}_{2}\right.$, hexanes/MTBE $\left.1: 4, \mathrm{R}_{\mathrm{f}}=0.23\right)$ as a colorless solid, mp. $57-59^{\circ} \mathrm{C}$, ref. ${ }^{\mathrm{S}} 56-59^{\circ} \mathrm{C} .{ }^{1} \mathrm{H} \mathrm{NMR}\left(300 \mathrm{MHz}, \mathrm{CDCl}_{3}\right): \delta=1.53$ (bs, $1 \mathrm{H}), 3.05$ (t, $J=6.4 \mathrm{~Hz}, 2 \mathrm{H}$ ), $3.92(\mathrm{t}, J=6.4 \mathrm{~Hz}, 2 \mathrm{H}), 7.10(\mathrm{~d}, J=1.9 \mathrm{~Hz}, 1 \mathrm{H}), 7.13(\mathrm{t}$, $J=7.4 \mathrm{~Hz}, 1 \mathrm{H}), 7.22(\mathrm{t}, J=7.1 \mathrm{~Hz}, 1 \mathrm{H}), 7.39(\mathrm{~d}, J=8.0 \mathrm{~Hz}, 1 \mathrm{H}), 7.63(\mathrm{~d}, J=7.8 \mathrm{~Hz}$, $1 \mathrm{H}), 8.03(\mathrm{bs}, 1 \mathrm{H}) \mathrm{ppm}$. All spectroscopic data are in accordance with the literature. ${ }^{\mathrm{s} 2}$ $\mathrm{C}_{10} \mathrm{H}_{11} \mathrm{NO}\left(161.20 \mathrm{~g} \mathrm{~mol}^{-1}\right)$.

5-Fluoro-3-(2-hydroxyethyl)indole (10b). According to the GP A, 4-fluorophenylhydrazine hydrochloride $(1.0 \mathrm{~g}, 6.2 \mathrm{mmol})$ and 2,3-dihydrofurane $(0.48 \mathrm{~g}, 6.8 \mathrm{mmol})$ were converted in $\mathrm{DMA} / \mathrm{H}_{2} \mathrm{SO}_{4} / \mathrm{H}_{2} \mathrm{O}(18 \mathrm{~mL})$ at $100^{\circ} \mathrm{C}$ for 90 min to furnish the product $10 \mathrm{~b}(0.71 \mathrm{~g}, 4.0 \mathrm{mmol}, 65 \%)$ after chromatography $\left(\mathrm{SiO}_{2}\right.$, hexanes/MTBE 1:6, $\mathrm{R}_{\mathrm{f}}=$ $0.26)$ as a colorless solid, mp. $58-60^{\circ} \mathrm{C}$, ref. ${ }^{\mathrm{S} 9} 59-61^{\circ} \mathrm{C} .{ }^{1} \mathrm{H}$ NMR $\left(300 \mathrm{MHz}, \mathrm{CDCl}_{3}\right): \delta$ $=1.66(\mathrm{~s}, 1 \mathrm{H}), 2.99(\mathrm{t}, J=6.3 \mathrm{~Hz}, 2 \mathrm{H}), 3.90(\mathrm{t}, J=6.3 \mathrm{~Hz}, 2 \mathrm{H}), 6.96$ (td, $J=9.0 \mathrm{~Hz}, J$ $=2.5 \mathrm{~Hz}, 1 \mathrm{H}), 7.10-7.16(\mathrm{~m}, 1 \mathrm{H}), 7.21-7.32(\mathrm{~m}, 2 \mathrm{H}), 8.08(\mathrm{~s}, 1 \mathrm{H})$. All spectroscopic data are in accordance with the literature. ${ }^{\mathrm{S3}} \mathrm{C}_{10} \mathrm{H}_{10} \mathrm{FNO}\left(179.19 \mathrm{~g} \mathrm{~mol}^{-1}\right)$.

5-Bromo-3-(2-hydroxyethyl)indole (10c). According to the GP A, 4-bromophenylhydrazine hydrochloride $(1.0 \mathrm{~g}, 4.5 \mathrm{mmol})$ and 2,3-dihydrofurane $(0.38 \mathrm{~g}, 5.4 \mathrm{mmol})$ were converted in $\mathrm{DMA} / \mathrm{H}_{2} \mathrm{SO}_{4} / \mathrm{H}_{2} \mathrm{O}(15 \mathrm{~mL})$ at $100^{\circ} \mathrm{C}$ for 90 min to furnish the product $10 \mathrm{c}(0.26 \mathrm{~g}, 1.1 \mathrm{mmol}, 24 \%)$ after chromatography $\left(\mathrm{SiO}_{2}\right.$, hexanes/MTBE 1:4, Rf $=$ $0.20)$ as a colorless oil. ${ }^{1} \mathrm{H}$ NMR $\left(300 \mathrm{MHz}, \mathrm{CDCl}_{3}\right): \delta=1.88(\mathrm{~s}, 1 \mathrm{H}), 2.96(\mathrm{t}, J=6.3$ $\mathrm{Hz}, 2 \mathrm{H}$ ), $3.88(\mathrm{t}, J=6.3 \mathrm{~Hz}, 2 \mathrm{H}), 7.03(\mathrm{~s}, 1 \mathrm{H}), 7.16-7.32(\mathrm{~m}, 2 \mathrm{H}), 7.73(\mathrm{~s}, 1 \mathrm{H}), 8.28$ (s, $1 \mathrm{H})$. All spectroscopic data are in accordance with the literature. ${ }^{S 4} \mathrm{C}_{10} \mathrm{H}_{10} \mathrm{BrNO}(240.10$ $\mathrm{g} \mathrm{mol}^{-1}$ ).

3-(2-Hydroxyethyl)-5-methoxyindole (10d). According to the GP A, 4-methoxyphenylhydrazine hydrochloride $(0.50 \mathrm{~g}, 2.9 \mathrm{mmol})$ and 2,3-dihydrofurane $(0.22 \mathrm{~g}, 3.2$ mmol) were converted in DMA $/ \mathrm{H}_{2} \mathrm{SO}_{4} / \mathrm{H}_{2} \mathrm{O}(10 \mathrm{~mL})$ at $100^{\circ} \mathrm{C}$ for $90 \mathrm{~min}$ to furnish the product $10 \mathrm{~d}(0.12 \mathrm{~g}, 0.63 \mathrm{mmol}, 22 \%)$ after chromatography $\left(\mathrm{SiO}_{2}\right.$, hexanes/MTBE $\left.1: 4, R_{f}=0.29\right)$ as a yellowish oil. ${ }^{1} \mathrm{H}$ NMR $\left(300 \mathrm{MHz}, \mathrm{CDCl}_{3}\right): \delta=1.58(\mathrm{~s}, 1 \mathrm{H}), 3.01(\mathrm{t}$, $J=6.4 \mathrm{~Hz}, 2 \mathrm{H}$ ), $3.87(\mathrm{~s}, 3 \mathrm{H}), 3.91(\mathrm{t}, J=6.4 \mathrm{~Hz}, 2 \mathrm{H}), 6.88(\mathrm{dd}, J=8.8 \mathrm{~Hz}, J=2.4 \mathrm{~Hz}$, 
$1 \mathrm{H}), 6.98(\mathrm{~d}, J=2.0 \mathrm{~Hz}, 1 \mathrm{H}), 7.07(\mathrm{~d}, J=2.3 \mathrm{~Hz}, 1 \mathrm{H}), 7.27(\mathrm{~d}, J=8.8 \mathrm{~Hz}, 1 \mathrm{H}), 8.25(\mathrm{~s}$, $1 \mathrm{H})$. All spectroscopic data are in accordance with the literature. ${ }^{S 7} \mathrm{C}_{11} \mathrm{H}_{13} \mathrm{NO}_{2}(191.23$ $\left.\mathrm{g} \mathrm{mol}^{-1}\right)$.

General Procedure B for the Oxa-Pictet-Spengler Reaction (GP B): TFA (1.2 eq.) was added to a solution of cyclohexanone 5 (1.0 eq.) and indole 10a-10d (1.0 eq.) in $\mathrm{CH}_{2} \mathrm{Cl}_{2}(4 \mathrm{~L} / \mathrm{mol})$. The resulting solution was stirred at $50^{\circ} \mathrm{C}$ for $16 \mathrm{~h}$. After cooling to $0^{\circ} \mathrm{C}$, aqueous $\mathrm{NaOH}(1 \mathrm{~mol} / \mathrm{L}, 4 \mathrm{~L} / \mathrm{mol})$ was added and the mixture was stirred for further $30 \mathrm{~min}$. The resulting precipitate was the trans-diastereoisomer $\mathbf{4 a - 4 d}$. It was filtered off, washed with $\mathrm{CH}_{2} \mathrm{Cl}_{2}$ and dried in vacuo. The filtrate contained cis- and trans-isomer. The aqueous layer was extracted with $\mathrm{CH}_{2} \mathrm{Cl}_{2}(3 \times 4 \mathrm{~L} / \mathrm{mol})$. The combined organic layers were dried $\left(\mathrm{MgSO}_{4}\right)$ and the solvent was evaporated in vacuo. The residue was chromatographed $\left(\mathrm{SiO}_{2}\right.$, hexanes/MTBE) to furnish cis-11a-11c and trans-4a-4d in separate fractions.

\section{trans-4-Nitro-4-phenyl-4',9'-dihydro-3' H-spiro[cyclohexane-1,1'-pyrano[3,4-b]in-} dole] (4a). According to the GP B, cyclohexanone $5(1.41 \mathrm{~g}, 6.45 \mathrm{mmol})$, indole 10a (1.04 g, $6.45 \mathrm{mmol}$ ) and TFA (883 mg, $7.74 \mathrm{mmol}$ ) were converted in $\mathrm{CH}_{2} \mathrm{Cl}_{2}(25 \mathrm{~mL})$ at $50^{\circ} \mathrm{C}$ for $16 \mathrm{~h}$ and further $30 \mathrm{~min}$ at $0^{\circ} \mathrm{C}$ with aqueous $\mathrm{NaOH}(1 \mathrm{~mol} / \mathrm{L}, 25 \mathrm{~mL})$ to furnish the product $4 \mathrm{a}(1.52 \mathrm{~g}, 4.20 \mathrm{mmol}, 65 \%)$ after chromatography $\left(\mathrm{SiO}_{2}\right.$, hexanes/MTBE $4: 1, R_{f}=0.53$ ) as a colorless solid, $\mathrm{mp} .222-224^{\circ} \mathrm{C}$. As the second fraction $\left(R_{f}=0.24\right)$, the cis-isomer 11a $(257 \mathrm{mg}, 0.71 \mathrm{mmol}, 11 \%)$ was obtained also as a colorless solid, mp. $243-245^{\circ} \mathrm{C} .{ }^{1} \mathrm{H} \mathrm{NMR}\left(500 \mathrm{MHz}, \mathrm{CDCl}_{3}\right)$ : $\delta=1.89$ (td, $J=14.4 \mathrm{~Hz}$, $J=3.6 \mathrm{~Hz}, 2 \mathrm{H}), 2.14-2.21(\mathrm{~m}, 2 \mathrm{H}), 2.51(\mathrm{td}, J=14.2 \mathrm{~Hz}, J=3.9 \mathrm{~Hz}, 2 \mathrm{H}), 2.82(\mathrm{t}, J=$ $5.4 \mathrm{~Hz}, 2 \mathrm{H}), 2.98-3.04(\mathrm{~m}, 2 \mathrm{H}), 4.00(\mathrm{t}, J=5.4 \mathrm{~Hz}, 2 \mathrm{H}), 7.10-7.15(\mathrm{~m}, 1 \mathrm{H}), 7.16-7.20$ $(\mathrm{m}, 1 \mathrm{H}), 7.33(\mathrm{~d}, J=5.0 \mathrm{~Hz}, 1 \mathrm{H}), 7.36-7.44(\mathrm{~m}, 3 \mathrm{H}), 7.48-7.55(\mathrm{~m}, 3 \mathrm{H}), 7.70(\mathrm{bs}, 1 \mathrm{H})$ ppm. ${ }^{13} \mathrm{C}\left\{{ }^{1} \mathrm{H}\right\}$ NMR $\left(125 \mathrm{MHz}, \mathrm{CDCl}_{3}\right): \delta=22.6\left(\mathrm{CH}_{2}\right), 30.2\left(2 \mathrm{CH}_{2}\right), 32.3\left(2 \mathrm{CH}_{2}\right), 60.4$ $\left(\mathrm{CH}_{2}\right), 70.8(\mathrm{C}), 92.8(\mathrm{C}), 107.8(\mathrm{C}), 111.2(\mathrm{CH}), 118.4(\mathrm{CH}), 119.9(\mathrm{CH}), 122.2(\mathrm{CH})$, $125.0(2 \mathrm{CH}), 127.0(\mathrm{C}), 129.1(2 \mathrm{CH}), 129.3(\mathrm{CH}), 135.9(\mathrm{C}), 137.4(\mathrm{C}), 140.0(\mathrm{C})$ ppm. IR (ATR): $3383(\mathrm{~m}), 2982(\mathrm{w}), 2879(\mathrm{w}), 1616(\mathrm{w}), 1533(\mathrm{~s}), 1496(\mathrm{w}), 1486(\mathrm{w})$, $1449(\mathrm{~m}), 1440(\mathrm{~m}), 1420(\mathrm{~m}), 1374(\mathrm{w}), 1353(\mathrm{~m}), 1340(\mathrm{~m}), 1316(\mathrm{~m}), 1299(\mathrm{~m}), 1266$ $(m), 1247(w), 1223(w), 1200(w), 1162(w), 1147(w), 1122(w), 1112(w), 1077(s)$, $1053(\mathrm{~m}), 1039(\mathrm{~m}), 1030(\mathrm{~m}), 1009(\mathrm{w}), 1002(\mathrm{w}), 964(\mathrm{~m}), 953(\mathrm{~m}), 936(\mathrm{~m}), 909(\mathrm{~m})$, $883(w), 850(m), 799(w), 763(s), 752(m), 729(w), 710(m), 690(s), 636(w), 610$ 
(m), $584(\mathrm{~m}), 563(\mathrm{~m}), 536(\mathrm{~m}) \mathrm{cm}^{-1} . \mathrm{MS}$ (El, $\left.70 \mathrm{eV}\right): \mathrm{m} / \mathrm{z}(\%) 362(4)[\mathrm{M}]^{+}, 316(14)$, 185 (100), 168 (10), 155 (62), 143 (15), 130 (14), 115 (32), 103 (10), 91 (30), 77 (16), 41 (20). HRMS (El, $70 \mathrm{eV}$ ) m/z: [M] ${ }^{+}$calcd. for $\mathrm{C}_{22} \mathrm{H}_{22} \mathrm{~N}_{2} \mathrm{O}_{3}{ }^{+} 362.1625$; found 362.1625 . $\mathrm{C}_{22} \mathrm{H}_{22} \mathrm{~N}_{2} \mathrm{O}_{3}\left(362.43 \mathrm{~g} \mathrm{~mol}^{-1}\right)$.

cis-4-Nitro-4-phenyl-4', 9' -dihydro-3' $H$-spiro[cyclohexane-1, 1'-pyrano[3,4-b]indole] (11a). The cis-isomer 11a (257 mg, $0.709 \mathrm{mmol}, 11 \%$ ) was obtained as second fraction according to the GP B as a colorless solid, mp. $243-245^{\circ} \mathrm{C}$. ${ }^{1} \mathrm{H} N M R(500 \mathrm{MHz}$, $\mathrm{CDCl}_{3}$ ): $\delta=1.67$ (td, $J=14.3 \mathrm{~Hz}, J=3.1 \mathrm{~Hz}, 2 \mathrm{H}$ ), 2.15 (d, $J=13.1 \mathrm{~Hz}, 2 \mathrm{H}$ ), 2.75 (td, $J=13.8 \mathrm{~Hz}, J=3.3 \mathrm{~Hz}, 2 \mathrm{H}), 2.81(\mathrm{t}, J=5.4 \mathrm{~Hz}, 2 \mathrm{H}), 3.04(\mathrm{~d}, J=13.5 \mathrm{~Hz}, 2 \mathrm{H}), 4.03(\mathrm{t}$, $J=5.4 \mathrm{~Hz}, 2 \mathrm{H}), 7.07-7.15(\mathrm{~m}, 2 \mathrm{H}), 7.17-7.21(\mathrm{~m}, 1 \mathrm{H}), 7.31$ (bs, 1H), 7.46-7.56 (m, $4 \mathrm{H}), 7.73-7.77(\mathrm{~m}, 2 \mathrm{H}) \mathrm{ppm} .{ }^{13} \mathrm{C}\left\{{ }^{1} \mathrm{H}\right\} \mathrm{NMR}\left(125 \mathrm{MHz}, \mathrm{CDCl}_{3}\right): \delta=22.5\left(\mathrm{CH}_{2}\right), 28.9(2$ $\left.\mathrm{CH}_{2}\right), 32.8\left(2 \mathrm{CH}_{2}\right), 60.2\left(\mathrm{CH}_{2}\right), 71.0(\mathrm{C}), 90.2(\mathrm{C}), 108.2(\mathrm{C}), 111.0(\mathrm{CH}), 118.4(\mathrm{CH})$, $119.9(\mathrm{CH}), 122.2(\mathrm{CH}), 127.0(\mathrm{C}), 127.6(2 \mathrm{CH}), 129.5(2 \mathrm{CH}), 129.8(\mathrm{CH}), 134.7(\mathrm{C})$, 135.7 (C), 136.9 (C) ppm. IR (ATR): 3225 (w), $3202(w), 2956(w), 2869(w), 1532(s)$, $1500(w), 1450(w), 1364(w), 1336(m), 1317(w), 1296(w), 1264(m), 1174(w), 1164$ (w), $1110(w), 1073(s), 1050(s), 1026(m), 1009(w), 954(m), 919(w), 886(w), 847$ (m), 747 (s), 710 (s), 694 (s), $619(\mathrm{~m}), 543(\mathrm{~m}) \mathrm{cm}^{-1}$. MS (El, $\left.70 \mathrm{eV}\right): \mathrm{m} / \mathrm{z}(\%) 362$ (32) [M]+, 316 (96), 185 (100), 155 (44), 144 (21), 115 (12), 91 (12). HRMS (El, $70 \mathrm{eV}), \mathrm{m} / \mathrm{z}$ : $[\mathrm{M}]^{+}$calcd. for $\mathrm{C}_{22} \mathrm{H}_{22} \mathrm{~N}_{2} \mathrm{O}_{3}{ }^{+} 362.1625$; found $362.1621 . \mathrm{C}_{22} \mathrm{H}_{22} \mathrm{~N}_{2} \mathrm{O}_{3}\left(362.43 \mathrm{~g} \mathrm{~mol}^{-1}\right)$.

\section{trans-6'-Fluoro-4-nitro-4-phenyl-4',9'-dihydro-3' $H$-spiro[cyclohexane-1,1'-pyra-}

no[3,4-b]indole] (4b). According to the GP B, cyclohexanone $5(3.00 \mathrm{~g}, 13.7 \mathrm{mmol})$, indole $10 \mathrm{~b}(2.45 \mathrm{mg}, 13.7 \mathrm{mmol})$ and TFA $(1.87 \mathrm{mg}, 16.4 \mathrm{mmol})$ were converted in $\mathrm{CH}_{2} \mathrm{Cl}_{2}(2 \mathrm{~mL})$ at $50^{\circ} \mathrm{C}$ for $16 \mathrm{~h}$ and further $30 \mathrm{~min}$ at $0^{\circ} \mathrm{C}$ with aqueous $\mathrm{NaOH}(1$ $\mathrm{mol} / \mathrm{L}, 20 \mathrm{~mL})$ to furnish the product $4 \mathrm{~b}(3.20 \mathrm{~g}, 8.44 \mathrm{mmol}, 62 \%)$ after chromatography $\left(\mathrm{SiO}_{2}\right.$, hexanes/MTBE 3:1, $\left.\mathrm{Rf}_{\mathrm{f}}=0.49\right)$ as a colorless solid, mp. $223-225^{\circ} \mathrm{C}$. As the second fraction $\left(R_{f}=0.18\right)$, the cis-isomer $11 b(124 \mathrm{mg}, 0.326 \mathrm{mmol}, 2 \%)$ was obtained also as a colorless solid, mp. $234-236^{\circ} \mathrm{C} .{ }^{1} \mathrm{H}$ NMR $\left(500 \mathrm{MHz}, \mathrm{DMSO}-\mathrm{d}_{6}\right): \delta=1.93-$ $2.06(\mathrm{~m}, 4 \mathrm{H}), 2.34$ (td, $J=14.2 \mathrm{~Hz}, J=4.5 \mathrm{~Hz}, 2 \mathrm{H}), 2.66(\mathrm{t}, J=5.3 \mathrm{~Hz}, 2 \mathrm{H}), 2.95(\mathrm{~d}, J$ $=13.7 \mathrm{~Hz}, 2 \mathrm{H}), 3.92(\mathrm{t}, J=5.4 \mathrm{~Hz}, 2 \mathrm{H}), 6.87(\mathrm{td}, J=9.2 \mathrm{~Hz}, J=2.5 \mathrm{~Hz}, 1 \mathrm{H}), 7.16(\mathrm{dd}$, $J=9.9 \mathrm{~Hz}, J=2.6 \mathrm{~Hz}, 1 \mathrm{H}), 7.26(\mathrm{dd}, J=8.8 \mathrm{~Hz}, J=4.5 \mathrm{~Hz}, 1 \mathrm{H}), 7.41-7.49(\mathrm{~m}, 3 \mathrm{H})$, 7.55-7.59 (m, 2H), $11.11(\mathrm{~s}, 1 \mathrm{H}) \mathrm{ppm} .{ }^{13} \mathrm{C}\left\{{ }^{1} \mathrm{H}\right\}$ NMR (125 MHz, DMSO-d 6$): \delta=22.0$ $\left(\mathrm{CH}_{2}\right), 29.4\left(2 \mathrm{CH}_{2}\right), 31.0\left(2 \mathrm{CH}_{2}\right), 59.4\left(\mathrm{CH}_{2}\right), 70.5(\mathrm{C}), 91.8(\mathrm{C}), 102.6(\mathrm{~d}, J=22 \mathrm{~Hz}$, 
$\mathrm{CH}), 105.9$ (d, J = $5 \mathrm{~Hz}, \mathrm{C}), 108.6$ (d, J = $26 \mathrm{~Hz}, \mathrm{CH}), 111.9$ (d, J = $10 \mathrm{~Hz}, \mathrm{CH}), 124.8$ (2 CH), 126.5 (d, J = $10 \mathrm{~Hz}, \mathrm{C}), 129.0$ (2 CH), $129.2(\mathrm{CH}), 132.3(\mathrm{C}), 139.8(\mathrm{C}), 140.4$ (C), 156.8 (d, J = $231 \mathrm{~Hz}, \mathrm{C}$ ) ppm. ${ }^{19} \mathrm{~F}\left\{{ }^{1} \mathrm{H}\right\}$ NMR (470 MHz, DMSO-d6): $\delta=-125.40$ (s) ppm. IR (ATR): 3378 (s), 2951 (w), 2867 (w), 1591 (w), 1534 (s) 1497 (w), 1488 (m), 1458 (w), 1448 (m), 1435 (m), 1421 (w), 1341 (w), 1307 (w), $1294(\mathrm{~m}), 1231$ (w), 1204 (w), $1178(w), 1144(\mathrm{~m}), 1128(\mathrm{w}), 1114(\mathrm{~m}), 1074(\mathrm{~s}), 1057(\mathrm{~m}), 1047(\mathrm{~m}), 1028(\mathrm{w})$, $970(w), 952(w), 922(w), 908(w), 838$ (s), $825(w), 801(s), 785(w), 757(m), 705(m)$, 688 (s), 677 (w), 638 (w), 608 (m), $602(\mathrm{~m}), 561(\mathrm{w}), 524(\mathrm{w}) \mathrm{cm}^{-1}$. MS (El, $\left.70 \mathrm{eV}\right): \mathrm{m} / \mathrm{z}$ (\%) 380 (14) [M]+, 334 (20), 203 (100), 173 (11), 155 (8), 91 (4). HRMS (EI, 70 eV) $\mathrm{m} / \mathrm{z}$ : [M] ${ }^{+}$calcd. for $\mathrm{C}_{22} \mathrm{H}_{21} \mathrm{FN}_{2} \mathrm{O}_{3}{ }^{+} 380.1531$; found 380.1542 . $\mathrm{C}_{22} \mathrm{H}_{21} \mathrm{FN}_{2} \mathrm{O}_{3}(380.42 \mathrm{~g}$ $\left.\mathrm{mol}^{-1}\right)$.

\section{cis-6'-Fluoro-4-nitro-4-phenyl-4',9'-dihydro-3' H-spiro[cyclohexane-1,1'-pyrano-}

[3,4-b]indole] (11b). The cis-isomer 11b (124 mg, $0.326 \mathrm{mmol}, 2 \%)$ was obtained as second fraction according to the GP B as a colorless solid, mp. $234-236^{\circ} \mathrm{C}$. ${ }^{1} \mathrm{H}$ NMR (500 MHz, DMSO-d6): $\delta=1.71-1.79(\mathrm{~m}, 2 \mathrm{H}), 2.00(\mathrm{~d}, J=13.2 \mathrm{~Hz}, 2 \mathrm{H}), 2.56$ (td, $J=$ $13.8 \mathrm{~Hz}, J=3.4 \mathrm{~Hz}, 2 \mathrm{H}), 2.66(\mathrm{t}, J=5.5 \mathrm{~Hz}, 2 \mathrm{H}), 2.99(\mathrm{~d}, J=13.2 \mathrm{~Hz}, 2 \mathrm{H}), 3.96$ (t, J $=5.4 \mathrm{~Hz}, 2 \mathrm{H}), 6.82(\mathrm{td}, J=9.1 \mathrm{~Hz}, J=2.6 \mathrm{~Hz}, 1 \mathrm{H}), 7.13(\mathrm{dd}, J=9.8 \mathrm{~Hz}, J=2.6 \mathrm{~Hz}$, $1 \mathrm{H}), 7.17(\mathrm{dd}, J=8.8 \mathrm{~Hz}, J=4.5 \mathrm{~Hz}, 1 \mathrm{H}), 7.50-7.58(\mathrm{~m}, 3 \mathrm{H}), 7.78-7.82(\mathrm{~m}, 2 \mathrm{H}), 10.78$ (s, 1H) ppm. ${ }^{13} \mathrm{C}\left\{{ }^{1} \mathrm{H}\right\}$ NMR (125 MHz, DMSO-d $): \delta=21.9\left(\mathrm{CH}_{2}\right), 28.3\left(2 \mathrm{CH}_{2}\right), 31.7(2$ $\left.\mathrm{CH}_{2}\right), 59.2\left(\mathrm{CH}_{2}\right), 70.8(\mathrm{C}), 90.8(\mathrm{C}), 102.6(\mathrm{~d}, \mathrm{~J}=23 \mathrm{~Hz}, \mathrm{CH}), 106.1$ (d, J = $\left.4 \mathrm{~Hz}, \mathrm{C}\right)$, $108.6(\mathrm{~d}, J=26 \mathrm{~Hz}, \mathrm{CH}), 111.9$ (d, $J=10 \mathrm{~Hz}, \mathrm{CH}), 126.5(\mathrm{~d}, J=10 \mathrm{~Hz}, \mathrm{C}), 127.4$ (2 $\mathrm{CH}), 129.1(2 \mathrm{CH}), 129.4(\mathrm{CH}), 132.3(\mathrm{C}), 134.7(\mathrm{C}), 139.8(\mathrm{C}), 156.7$ (d, J = $231 \mathrm{~Hz}$, C) ppm. ${ }^{19} \mathrm{~F}\left\{{ }^{1} \mathrm{H}\right\} \mathrm{NMR}\left(470 \mathrm{MHz}, \mathrm{DMSO}-\mathrm{d}_{6}\right): \delta=-125.40$ (s) ppm. IR (ATR): 3360 (m), 2944 (w), 2855 (w), 1590 (w), 1525 (s), $1488(\mathrm{~m}), 1452(\mathrm{~m}), 1362(\mathrm{~m}), 1335(\mathrm{w}), 1311$ (w), $1294(\mathrm{~m}), 1267(\mathrm{w}), 1232(\mathrm{w}), 1208(\mathrm{w}), 1170(\mathrm{w}), 1141(\mathrm{~m}), 1108(\mathrm{w}), 1082(\mathrm{~m})$, $1075(\mathrm{~s}), 1055(\mathrm{~m}), 1024(\mathrm{w}), 955(\mathrm{w}), 918(\mathrm{w}), 850(\mathrm{~m}), 838(\mathrm{~s}), 827(\mathrm{w}), 800(\mathrm{~s}), 787$ $(\mathrm{m}), 751(\mathrm{~m}), 710(\mathrm{~s}), 692(\mathrm{~m}), 670(\mathrm{w}), 618(\mathrm{~m}), 604(\mathrm{~m}), 560(\mathrm{w}), 535(\mathrm{w}) \mathrm{cm}^{-1}$. HRMS (ESI, neg. mode) $\mathrm{m} / \mathrm{z}$ : $\left[\mathrm{M}-\mathrm{H}^{-}\right.$calcd. for $\mathrm{C}_{22} \mathrm{H}_{20} \mathrm{FN}_{2} \mathrm{O}_{3}-379.1463$; found 379.1479. $\mathrm{C}_{22} \mathrm{H}_{21} \mathrm{FN}_{2} \mathrm{O}_{3}\left(380.42 \mathrm{~g} \mathrm{~mol}^{-1}\right)$.

\section{trans-6'-Bromo-4-nitro-4-phenyl-4',9'-dihydro-3' H-spiro[cyclohexane-1,1'-pyra-}

no[3,4-b]indole] (4c). According to the GP B, cyclohexanone 5 (137 mg, $0.625 \mathrm{mmol}$ ), indole 10c (150 mg, $0.625 \mathrm{mmol})$ and TFA ( $86 \mathrm{mg}, 0.75 \mathrm{mmol}$ ) were converted in 
$\mathrm{CH}_{2} \mathrm{Cl}_{2}(10 \mathrm{~mL})$ at $50^{\circ} \mathrm{C}$ for $16 \mathrm{~h}$ and further $30 \mathrm{~min}$ at $0^{\circ} \mathrm{C}$ with aqueous $\mathrm{NaOH}$ (1 $\mathrm{mol} / \mathrm{L}, 10 \mathrm{~mL}$ ) to furnish the product $4 \mathrm{c}(92 \mathrm{mg}, 0.21 \mathrm{mmol}, 34 \%)$ after chromatography $\left(\mathrm{SiO}_{2}\right.$, hexanes/MTBE $\left.2: 1, \mathrm{R}_{\mathrm{f}}=0.56\right)$ as a colorless solid, mp. $229-231^{\circ} \mathrm{C}$. As the second fraction $\left(R_{f}=0.27\right)$, the cis-isomer $11 \mathrm{c}(16 \mathrm{mg}, 0.036 \mathrm{mmol}, 6 \%)$ was obtained also as a colorless solid, mp. $251-253^{\circ} \mathrm{C}$. ${ }^{1} \mathrm{H}$ NMR $(500 \mathrm{MHz}$, DMSO-d 6 ): $\delta=1.89-$ $2.06(\mathrm{~m}, 4 \mathrm{H}), 2.34(\mathrm{t}, J=11.2 \mathrm{~Hz}, 2 \mathrm{H}), 2.68(\mathrm{~s}, 2 \mathrm{H}), 2.95(\mathrm{~d}, J=13.7 \mathrm{~Hz}, 2 \mathrm{H}), 3.92(\mathrm{~s}$, $2 \mathrm{H}), 7.11-7.20(\mathrm{~m}, 1 \mathrm{H}), 7.20-7.29(\mathrm{~m}, 1 \mathrm{H}), 7.38-7.50(\mathrm{~m}, 3 \mathrm{H}), 7.51-7.65(\mathrm{~m}, 3 \mathrm{H})$, 11.24 (s, 1H) ppm. ${ }^{13} \mathrm{C}\left\{{ }^{1} \mathrm{H}\right\}$ NMR (125 MHz, DMSO-d 6$): \delta=21.9\left(\mathrm{CH}_{2}\right), 29.4\left(2 \mathrm{CH}_{2}\right)$, $31.0\left(2 \mathrm{CH}_{2}\right), 59.4\left(\mathrm{CH}_{2}\right), 70.5(\mathrm{C}), 91.8(\mathrm{C}), 105.5(\mathrm{C}), 111.0(\mathrm{C}), 113.1(\mathrm{CH}), 120.2$ $(\mathrm{CH}), 123.2(\mathrm{CH}), 124.8(2 \mathrm{CH}), 128.2(\mathrm{C}), 129.0(2 \mathrm{CH}), 129.2(\mathrm{CH}), 134.4(\mathrm{C}), 139.7$ (C), 139.9 (C) ppm. IR (ATR): $3368(\mathrm{~m}), 2955$ (w), 2925 (w), 2867 (w), $1532(\mathrm{~s}), 1497$ (w), $1475(w), 1464(w), 1448(m), 1435(m), 1421(m), 1375(w), 1350(w), 1300(m)$, $1287(\mathrm{~m}), 1245(\mathrm{w}), 1234(\mathrm{w}), 1200(\mathrm{w}), 1160(\mathrm{w}), 1124(\mathrm{w}), 1075(\mathrm{~s}), 1060(\mathrm{~s}), 1030$ (w), $965(w), 954(w), 912(w), 888(w), 871(w), 851(m), 797(s), 757(m), 740(w)$, $705(\mathrm{~m}), 690(\mathrm{~m}), 677(\mathrm{w}), 652(\mathrm{w}), 632(\mathrm{w}), 597(\mathrm{w}), 587(\mathrm{~m}), 550(\mathrm{w}) \mathrm{cm}^{-1}$. HRMS (ESI, neg. mode) $\mathrm{m} / \mathrm{z}$ : $\left[\mathrm{M}-\mathrm{H}^{-}\right.$calcd. for $\mathrm{C}_{22} \mathrm{H}_{20} \mathrm{BrN}_{2} \mathrm{O}_{3}{ }^{-} 439.0663$; found 439.0645 . $\mathrm{C}_{22} \mathrm{H}_{21} \mathrm{BrN}_{2} \mathrm{O}_{3}\left(441.33 \mathrm{~g} \mathrm{~mol}^{-1}\right)$.

\section{cis-6'-Bromo-4-nitro-4-phenyl-4',9'-dihydro-3' $H$-spiro[cyclohexane-1,1'-pyrano-}

[3,4-b]indole] (11c). The cis-isomer 11c (16 mg, $0.036 \mathrm{mmol}, 6 \%)$ was obtained as second fraction according to the GP B as a colorless solid, mp. $251-253^{\circ} \mathrm{C} .{ }^{1} \mathrm{H}$ NMR (500 MHz, DMSO-d 6 ): $\delta=1.76$ (td, $J=13.9 \mathrm{~Hz}, J=2.8 \mathrm{~Hz}, 2 \mathrm{H}$ ), 2.00 (d, $J=13.0 \mathrm{~Hz}$, 2H), 2.57 (td, $J=13.6 \mathrm{~Hz}, J=3.3 \mathrm{~Hz}, 2 \mathrm{H}$ ), 2.68 (t, $J=5.4 \mathrm{~Hz}, 2 \mathrm{H}$ ), 2.99 (d, $J=13.3$ $\mathrm{Hz}, 2 \mathrm{H}$ ), $3.96(\mathrm{t}, J=5.4 \mathrm{~Hz}, 2 \mathrm{H}), 7.11(\mathrm{dd}, J=8.6 \mathrm{~Hz}, J=2.0 \mathrm{~Hz}, 1 \mathrm{H}), 7.16(\mathrm{~d}, J=8.5$ $\mathrm{Hz}, 1 \mathrm{H}), 7.50-7.59(\mathrm{~m}, 4 \mathrm{H}), 7.79-7.83(\mathrm{~m}, 2 \mathrm{H}), 10.92(\mathrm{~s}, 1 \mathrm{H}) \mathrm{ppm} .{ }^{13} \mathrm{C}\left\{{ }^{1} \mathrm{H}\right\} \mathrm{NMR}(125$ $\mathrm{MHz}$, DMSO-d $)$ : $\delta=21.8\left(\mathrm{CH}_{2}\right), 28.3\left(2 \mathrm{CH}_{2}\right), 31.6\left(2 \mathrm{CH}_{2}\right), 59.2\left(\mathrm{CH}_{2}\right), 70.8(\mathrm{C}), 90.7$ (C), $105.7(\mathrm{C}), 111.0(\mathrm{C}), 113.0(\mathrm{CH}), 120.1(\mathrm{CH}), 123.2(\mathrm{CH}), 127.4(2 \mathrm{CH}), 128.2(\mathrm{C})$, $129.1(2 \mathrm{CH}), 129.4(\mathrm{CH}), 134.4(\mathrm{C}), 134.7(\mathrm{C}), 139.4$ (C) ppm. IR (ATR): $3371(\mathrm{~m})$, $2948(w), 2918(w), 2840(w), 1530(s), 1498(w), 1478(m), 1450(m), 1435(m), 1360$ $(m), 1334(w), 1307(m), 1298(w), 1288(m), 1270(w), 1208(w), 1174(w), 1161(w)$, $1108(\mathrm{w}), 1077(\mathrm{~s}), 1058(\mathrm{~s}), 1025(\mathrm{~m}), 955(\mathrm{~m}), 890(\mathrm{w}), 852(\mathrm{~m}), 797(\mathrm{~s}), 751(\mathrm{~s}), 710$ $(\mathrm{s}), 695(\mathrm{~m}), 668(\mathrm{w}), 652(\mathrm{w}), 610(\mathrm{~m}), 587(\mathrm{~m}), 551(\mathrm{w}) \mathrm{cm}^{-1}$. HRMS (ESI, neg. mode) $\mathrm{m} / \mathrm{z}$ : $[\mathrm{M}-\mathrm{H}]^{-}$calcd. for $\mathrm{C}_{22} \mathrm{H}_{20} \mathrm{BrN}_{2} \mathrm{O}_{3}-439.0663$; found 439.0645. $\mathrm{C}_{22} \mathrm{H}_{21} \mathrm{BrN}_{2} \mathrm{O}_{3}$ $\left(441.33 \mathrm{~g} \mathrm{~mol}^{-1}\right)$. 
trans-6'-Methoxy-4-nitro-4-phenyl-4',9'-dihydro-3' H-spiro[cyclohexane-1,1'-pyrano[3,4-b]indole] (4d). According to the GP B, cyclohexanone 5 (129 mg, $0.588 \mathrm{mmol}$ ), indole $10 \mathrm{~d}(112 \mathrm{mg}, 0.588 \mathrm{mmol})$ and TFA (81 $\mathrm{mg}, 0.71 \mathrm{mmol})$ were converted in $\mathrm{CH}_{2} \mathrm{Cl}_{2}(5 \mathrm{~mL})$ at $50^{\circ} \mathrm{C}$ for $16 \mathrm{~h}$ and further $30 \mathrm{~min}$ at $0^{\circ} \mathrm{C}$ with aqueous $\mathrm{NaOH}(1 \mathrm{~mol} / \mathrm{L}$, $5 \mathrm{~mL}$ ) to furnish the product $\mathbf{4 d}(104 \mathrm{mg}, 0.265 \mathrm{mmol}, 46 \%)$ after chromatography $\left(\mathrm{SiO}_{2}\right.$, hexanes/MTBE 2:1, $\left.\mathrm{R}_{\mathrm{f}}=0.44\right)$ as a colorless solid, $\mathrm{mp} .227-229^{\circ} \mathrm{C}$. A cis-isomer 11d was not observed. ${ }^{1} \mathrm{H}$ NMR $\left(500 \mathrm{MHz}, \mathrm{CDCl}_{3}\right): \delta=1.88(\mathrm{td}, J=14.1 \mathrm{~Hz}, J=3.6$ $\mathrm{Hz}, 2 \mathrm{H}), 2.17(\mathrm{~d}, J=14.1 \mathrm{~Hz}, 2 \mathrm{H}), 2.50(\mathrm{td}, J=14.1 \mathrm{~Hz}, J=4.0 \mathrm{~Hz}, 2 \mathrm{H}), 2.79(\mathrm{t}, J=$ $5.4 \mathrm{~Hz}, 2 \mathrm{H}$ ), 3.00 (d, J = $13.6 \mathrm{~Hz}, 2 \mathrm{H}$ ), $3.86(\mathrm{~s}, 3 \mathrm{H}), 4.00$ (t, J = 5.4 Hz, 2H), 6.83 (dd, $J=8.8 \mathrm{~Hz}, J=2.5 \mathrm{~Hz}, 1 \mathrm{H}), 6.95(\mathrm{~d}, J=2.4 \mathrm{~Hz}, 1 \mathrm{H}), 7.20(\mathrm{~d}, J=8.7 \mathrm{~Hz}, 1 \mathrm{H}), 7.36-$ $7.44(\mathrm{~m}, 3 \mathrm{H}), 7.50-7.54(\mathrm{~m}, 2 \mathrm{H}), 7.60$ (bs, $1 \mathrm{H}) \mathrm{ppm} .{ }^{13} \mathrm{C}\left\{{ }^{1} \mathrm{H}\right\}$ NMR $\left(125 \mathrm{MHz}, \mathrm{CDCl}_{3}\right)$ : $\delta=22.6\left(\mathrm{CH}_{2}\right), 30.1\left(2 \mathrm{CH}_{2}\right), 32.3\left(2 \mathrm{CH}_{2}\right), 56.2\left(\mathrm{CH}_{3}\right), 60.4\left(\mathrm{CH}_{2}\right), 70.9(\mathrm{C}), 92.8(\mathrm{C})$, $100.7(\mathrm{CH}), 107.6(\mathrm{C}), 111.9(\mathrm{CH}), 112.0(\mathrm{CH}), 125.0(2 \mathrm{CH}), 127.4(\mathrm{C}), 129.1(2 \mathrm{CH})$, $129.3(\mathrm{CH}), 131.0$ (C), 138.3 (C), 140.0 (C), 154.4 (C) ppm. IR (ATR): 3450 (w), 3390 (w), $2923(\mathrm{~m}), 2854(\mathrm{w}), 2840(\mathrm{w}), 1590(\mathrm{w}), 1537$ (s), 1532 (s), $1482(\mathrm{~m}), 1467(\mathrm{~m})$, $1455(\mathrm{~m}), 1448(\mathrm{~m}), 1432(\mathrm{~m}), 1420(\mathrm{w}), 1344(\mathrm{w}), 1294(\mathrm{~m}), 1264(\mathrm{w}), 1215(\mathrm{~m}), 1204$ (m), $1181(\mathrm{w}), 1145(\mathrm{~m}), 1071(\mathrm{~s}), 1045(\mathrm{~m}), 1022(\mathrm{~m}), 950(\mathrm{~m}), 907(\mathrm{~m}), 870(\mathrm{w}), 845$ $(\mathrm{m}), 831(\mathrm{~m}), 807(\mathrm{w}), 789(\mathrm{~m}), 777(\mathrm{w}), 760(\mathrm{~s}), 710(\mathrm{~s}), 691(\mathrm{~m}), 675(\mathrm{w}), 640(\mathrm{w})$, 621 (w), 604 (w), 580 (w), 542 (w), 531 (w) cm-1. MS (El, $70 \mathrm{eV}): \mathrm{m} / \mathrm{z}$ (\%) 392 (12) $[\mathrm{M}]^{+}, 346$ (30), 215 (100), 185 (10). HRMS (El, $70 \mathrm{eV}$ ) m/z: [M] calcd. $\mathrm{C}_{23} \mathrm{H}_{24} \mathrm{~N}_{2} \mathrm{O}_{4}{ }^{+}$ 392.1731; found 392.1738. $\mathrm{C}_{23} \mathrm{H}_{24} \mathrm{~N}_{2} \mathrm{O}_{4}\left(392.46 \mathrm{~g} \mathrm{~mol}^{-1}\right)$.

\section{trans-4-Amino-4-phenyl-4',9'-dihydro-3' H-spiro[cyclohexane-1,1'-pyrano[3,4-b]-}

indole] (12). A solution of indole $4 \mathrm{a}(64 \mathrm{mg}, 0.18 \mathrm{mmol})$, zinc powder $(0.47 \mathrm{~g}, 7.2$ $\mathrm{mmol}$ ) and formic acid $(0.33 \mathrm{~g}, 7.2 \mathrm{mmol})$ in $\mathrm{MeOH}(10 \mathrm{~mL})$ was stirred $16 \mathrm{~h}$ at $50^{\circ} \mathrm{C}$. After cooling to ambient temperature, the solution was diluted with saturated, aqueous $\mathrm{NaHCO}_{3}$ solution $(15 \mathrm{~mL})$ and extracted with EtOAc $(3 \times 20 \mathrm{~mL})$. The organic layers were dried $\left(\mathrm{MgSO}_{4}\right)$, filtered and the solvent was evaporated in vacuo to furnish the amine 12 (53 mg, $0.16 \mathrm{mmol}, 89 \%)$ as a colorless solid, mp. 221-223 ${ }^{\circ} \mathrm{C}$. ${ }^{1} \mathrm{H}$ NMR (500 $\left.\mathrm{MHz}, \mathrm{CDCl}_{3}\right): \delta=1.95-2.13(\mathrm{~m}, 4 \mathrm{H}), 2.31-2.42(\mathrm{~m}, 4 \mathrm{H}), 2.82(\mathrm{t}, J=5.3 \mathrm{~Hz}, 2 \mathrm{H}), 3.96$ $(\mathrm{t}, J=5.3 \mathrm{~Hz}, 2 \mathrm{H}), 5.63(\mathrm{bs}, 2 \mathrm{H}), 7.06-7.12(\mathrm{~m}, 2 \mathrm{H}), 7.18-7.29(\mathrm{~m}, 4 \mathrm{H}), 7.48-7.53(\mathrm{~m}$, $1 \mathrm{H}), 7.60-7.65(\mathrm{~m}, 2 \mathrm{H}), 9.42(\mathrm{~s}, 1 \mathrm{H}) \mathrm{ppm} .{ }^{13} \mathrm{C}\left\{{ }^{1} \mathrm{H}\right\} \mathrm{NMR}\left(125 \mathrm{MHz}, \mathrm{CDCl}_{3}\right): \delta=22.7$ $\left(\mathrm{CH}_{2}\right), 30.8\left(2 \mathrm{CH}_{2}\right), 31.5\left(2 \mathrm{CH}_{2}\right), 56.8(\mathrm{C}), 60.2\left(\mathrm{CH}_{2}\right), 71.2(\mathrm{C}), 106.9(\mathrm{C}), 111.3(\mathrm{CH})$, 
$118.2(\mathrm{CH}), 119.3(\mathrm{CH}), 121.6(\mathrm{CH}), 125.1(2 \mathrm{CH}), 126.8(\mathrm{C}), 128.2(\mathrm{CH}), 128.9(2$ CH), 135.9 (C), 138.3 (C), 144.5 (C) ppm. IR (ATR): 3385 (m), 2947 (w), 2923 (w), $2850(w), 1579(w), 1447(w), 1374(w), 1362(w), 1316(w), 1299(m), 1272(m), 1243$ (w), $1223(w), 1200(w), 1159(w), 1074(s), 1050(s), 1030(m), 950(w), 912(w), 866$ (w), $816(w), 756(s), 696(s), 676(w), 643(w), 583(w), 543(s), 523(w) \mathrm{cm}^{-1}$. MS (EI, $70 \mathrm{eV}): \mathrm{m} / z$ (\%) 332 (18) [M] ${ }^{+}, 315$ (21), 287 (10), 200 (10), 185 (100), 155 (12). HRMS (El, $70 \mathrm{eV}$ ) m/z: [M] $]^{+}$calcd. for $\mathrm{C}_{22} \mathrm{H}_{24} \mathrm{~N}_{2} \mathrm{O}^{+}$332.1883; found 332.1883. $\mathrm{C}_{22} \mathrm{H}_{24} \mathrm{~N}_{2} \mathrm{O}$ $\left(332.45 \mathrm{~g} \mathrm{~mol}^{-1}\right)$.

\section{trans-4-(Dimethylamino)-4-phenyl-4',9'-dihydro-3' $\mathrm{H}$-spiro[cyclohexane-1,1'-}

pyrano[3,4-b]indole] (1a). A solution of indole $4 \mathrm{a}(0.10 \mathrm{~g}, 0.28 \mathrm{mmol})$, zinc dust (732 $\mathrm{mg}, 11.2 \mathrm{mmol}$ ) and formic acid (516 mg, $11.2 \mathrm{mmol})$ in $\mathrm{MeOH}(10 \mathrm{~mL})$ was stirred 16 $\mathrm{h}$ at $50^{\circ} \mathrm{C}$. After cooling to ambient temperature, formic acid (516 mg, $\left.11.2 \mathrm{mmol}\right)$ and formaldehyde ( $37 \%$ aqueous solution, $920 \mathrm{mg}, 11.2 \mathrm{mmol}$ ) were added. The suspension was further stirred at $70^{\circ} \mathrm{C}$ for $16 \mathrm{~h}$. After cooling to ambient temperature, the solution was diluted with saturated, aqueous $\mathrm{NaHCO}_{3}$ solution $(15 \mathrm{~mL})$ and extracted with EtOAc $(3 \times 20 \mathrm{~mL})$. The organic layers were dried $\left(\mathrm{MgSO}_{4}\right)$, filtered and the solvent was evaporated in vacuo. The residue was chromatographed $\left(\mathrm{SiO}_{2}, \mathrm{MTBE}, \mathrm{R}_{\mathrm{f}}=0.17\right)$ to furnish amine $1 \mathrm{a}(63 \mathrm{mg}, 0.18 \mathrm{mmol}, 64 \%)$ as a colorless solid, mp. $269-271^{\circ} \mathrm{C} .{ }^{1} \mathrm{H}$ NMR (500 MHz, CDCl $)$ : $\delta=1.89-1.96(\mathrm{~m}, 2 \mathrm{H}), 2.02(\mathrm{td}, J=13.7 \mathrm{~Hz}, J=2.8 \mathrm{~Hz}, 2 \mathrm{H})$, $2.13(\mathrm{~s}, 6 \mathrm{H}), 2.25(\mathrm{td}, J=13.7 \mathrm{~Hz}, J=3.1 \mathrm{~Hz}, 2 \mathrm{H}), 2.49-2.56(\mathrm{~m}, 2 \mathrm{H}), 2.81(\mathrm{t}, J=5.4$ $\mathrm{Hz}, 2 \mathrm{H}), 3.98(\mathrm{t}, J=5.4 \mathrm{~Hz}, 2 \mathrm{H}), 7.08-7.13(\mathrm{~m}, 1 \mathrm{H}), 7.14-7.18(\mathrm{~m}, 1 \mathrm{H}), 7.26-7.31(\mathrm{~m}$, $1 \mathrm{H}), 7.35-7.40(\mathrm{~m}, 5 \mathrm{H}), 7.50(\mathrm{~d}, J=7.6 \mathrm{~Hz}, 1 \mathrm{H}), 8.37(\mathrm{~s}, 1 \mathrm{H}) \mathrm{ppm} .{ }^{13} \mathrm{C}\left\{{ }^{1} \mathrm{H}\right\} \mathrm{NMR}(125$ $\left.\mathrm{MHz}, \mathrm{CDCl}_{3}\right): \delta=22.8\left(\mathrm{CH}_{2}\right), 28.5\left(2 \mathrm{CH}_{2}\right), 31.2\left(2 \mathrm{CH}_{2}\right), 38.4\left(2 \mathrm{CH}_{3}\right), 58.9(\mathrm{C}), 60.0$ $\left(\mathrm{CH}_{2}\right), 72.3(\mathrm{C}), 107.2(\mathrm{C}), 111.1(\mathrm{CH}), 118.3(\mathrm{CH}), 119.5(\mathrm{CH}), 121.6(\mathrm{CH}), 126.8$ (CH), $127.1(2 \mathrm{CH}), 127.2(\mathrm{C}), 127.6(2 \mathrm{CH}), 135.9(\mathrm{C}), 139.4(\mathrm{C}), 139.4(\mathrm{C}) \mathrm{ppm}$. IR (ATR): $3377(m), 3272(m), 2923(m), 2856(w), 2782(w), 1582(w), 1452(m), 1372$ $(w), 1314(w), 1297(m), 1267(m), 1229(w), 1197(w), 1159(w), 1079(s), 1069(s)$, $1046(\mathrm{~s}), 1010$ (w), $913(\mathrm{w}), 869$ (w), 760 (s), $743(\mathrm{~s}), 701(\mathrm{~s}), 669(\mathrm{~m}), 610(\mathrm{w}), 579$ (w), $554(\mathrm{~m}), 530$ (w) cm-1. MS (El, $70 \mathrm{eV}): \mathrm{m} / \mathrm{z}(\%) 360$ (5) [M] $]^{+}, 315$ (12), 185 (100), 155 (36), 146 (12), 115 (12), 91 (13), 44 (44). HRMS (El, $70 \mathrm{eV}$ ) m/z: [M] calcd. for $\mathrm{C}_{24} \mathrm{H}_{28} \mathrm{~N}_{2} \mathrm{O}^{+} 360.2196$; found 360.2183 . $\mathrm{C}_{24} \mathrm{H}_{28} \mathrm{~N}_{2} \mathrm{O}$ (360.50 $\left.\mathrm{g} \mathrm{mol}^{-1}\right)$. 
trans-4-(Dimethylamino)-6'-fluoro-4-phenyl-4',9'-dihydro-3' $H$-spiro[cyclohexane1,1'-pyrano[3,4-b]indole] (1b). A solution of indole $4 \mathrm{~b}(0.34 \mathrm{~g}, 0.89 \mathrm{mmol})$, zinc dust $(2.33 \mathrm{~g}, 35.6 \mathrm{mmol})$ and formic acid $(1.64 \mathrm{~g}, 35.6 \mathrm{mmol})$ in $\mathrm{MeOH}(20 \mathrm{~mL})$ was stirred $16 \mathrm{~h}$ at $50^{\circ} \mathrm{C}$. After cooling to ambient temperature, formic acid $(1.64 \mathrm{~g}, 35.6 \mathrm{mmol})$ and formaldehyde ( $37 \%$ aqueous solution, $2.90 \mathrm{~g}, 35.8 \mathrm{mmol}$ ) were added. The suspension was further stirred at $70^{\circ} \mathrm{C}$ for $16 \mathrm{~h}$. After cooling to ambient temperature, the solution was diluted with saturated, aqueous $\mathrm{NaHCO}_{3}$ solution $(30 \mathrm{~mL})$ and extracted with EtOAc $(3 \times 30 \mathrm{~mL})$. The organic layers were dried $\left(\mathrm{MgSO}_{4}\right)$, filtered and the solvent was evaporated in vacuo. The residue was washed with MTBE (50 mL) to furnish amine $1 \mathrm{~b}(280 \mathrm{mg}, 0.740 \mathrm{mmol}, 83 \%)$ as a colorless solid, $\mathrm{mp} .264-266^{\circ} \mathrm{C}$, ref. $\mathrm{S} 10$ 279-284 ${ }^{\circ} \mathrm{C} .{ }^{1} \mathrm{H}$ NMR (500 MHz, DMSO-d $): \delta=1.71-1.81(\mathrm{~m}, 4 \mathrm{H}), 2.06(\mathrm{~s}, 6 \mathrm{H}), 2.26$ $(\mathrm{t}, J=12.2 \mathrm{~Hz}, 2 \mathrm{H}), 2.50-2.56(\mathrm{~m}, 2 \mathrm{H}), 2.64(\mathrm{t}, J=5.4 \mathrm{~Hz}, 2 \mathrm{H}), 3.88(\mathrm{t}, J=5.4 \mathrm{~Hz}$, $2 \mathrm{H}$ ), 6.83-6.85 (ddd, $J=9.6 \mathrm{~Hz}, J=8.8 \mathrm{~Hz}, J=2.6 \mathrm{~Hz}, 1 \mathrm{H}), 7.12(\mathrm{dd}, J=9.9 \mathrm{~Hz}, J=$ $2.5 \mathrm{~Hz}, 1 \mathrm{H}), 7.24-7.28(\mathrm{~m}, 1 \mathrm{H}), 7.30(\mathrm{dd}, J=8.8 \mathrm{~Hz}, J=4.6 \mathrm{~Hz}, 1 \mathrm{H}), 7.35-7.38(\mathrm{~m}$, 4H), $10.94(\mathrm{~s}, 1 \mathrm{H}) \mathrm{ppm} .{ }^{13} \mathrm{C}\left\{{ }^{1} \mathrm{H}\right\}$ NMR $\left(125 \mathrm{MHz}, \mathrm{DMSO}-\mathrm{d}_{6}\right): \delta=22.1\left(\mathrm{CH}_{2}\right), 27.8(2$ $\left.\mathrm{CH}_{2}\right), 30.4\left(2 \mathrm{CH}_{2}\right), 38.0\left(2 \mathrm{CH}_{3}\right), 57.7(\mathrm{C}), 58.8\left(\mathrm{CH}_{2}\right), 71.8(\mathrm{C}), 102.3(\mathrm{~d}, \mathrm{~J}=23 \mathrm{~Hz}$, $\mathrm{CH}), 105.5$ (d, J = $4 \mathrm{~Hz}, \mathrm{C}), 108.2$ (d, J = $26 \mathrm{~Hz}, \mathrm{CH}), 112.0$ (d, J = $10 \mathrm{~Hz}, \mathrm{CH}), 126.2$ $(\mathrm{CH}), 126.4(2 \mathrm{CH}), 126.6(\mathrm{~d}, J=10 \mathrm{~Hz}, \mathrm{C}), 127.2(2 \mathrm{CH}), 132.5(\mathrm{C}), 139.6(\mathrm{C}), 141.7$ (C), 156.7 (d, J = $231 \mathrm{~Hz}, \mathrm{C}) \mathrm{ppm} .{ }^{19} \mathrm{~F}\left\{{ }^{1} \mathrm{H}\right\} \mathrm{NMR}\left(470 \mathrm{MHz}, \mathrm{DMSO}-\mathrm{d}_{6}\right): \delta=-125.62$ (s) ppm. IR (ATR): 3385 (m), 3290 (w), 2947 (w), 2921 (w), 2867 (w), 1582 (m), 1495 (m), 1485 (m), 1451 (s), 1417 (w), 1371 (w), 1308 (w), 1288 (m), $1264(w), 1227(w), 1200$ (w), $1174(\mathrm{~m}), 1142(\mathrm{~m}), 1117(\mathrm{w}), 1110(\mathrm{w}), 1077(\mathrm{~m}), 1065(\mathrm{~m}), 1055(\mathrm{w}), 1042(\mathrm{~m})$, $1028(\mathrm{~m}), 987(\mathrm{~m}), 981(\mathrm{~m}), 920(\mathrm{w}), 910(\mathrm{w}), 870(\mathrm{~m}), 858(\mathrm{~s}), 825(\mathrm{w}), 820(\mathrm{w}), 792$ (m), $785(\mathrm{w}), 760$ (s), 701 (s), $670(\mathrm{w}), 634(\mathrm{w}), 604(\mathrm{~m}), 554(\mathrm{~m}), 518(\mathrm{~m}) \mathrm{cm}^{-1}$. HRMS (ESI, neg. mode) $\mathrm{m} / \mathrm{z}$ : $[\mathrm{M}-\mathrm{H}]^{-}$calcd. for $\mathrm{C}_{24} \mathrm{H}_{26} \mathrm{FN}_{2} \mathrm{O}^{-} 377.2035$; found 377.2047 . $\mathrm{C}_{24} \mathrm{H}_{27} \mathrm{FN} 2 \mathrm{O}\left(378.49 \mathrm{~g} \mathrm{~mol}^{-1}\right)$.

\section{trans-4-(N-Acetylamino)-4-phenyl-4',9'-dihydro-3' H-spiro[cyclohexane-1,1'-pyra-} no[3,4-b]indole] (13). A solution of amine $12(0.10 \mathrm{~g}, 0.30 \mathrm{mmol})$ and acetic anhydride (37 mg, $0.36 \mathrm{mmol})$ in THF $(5 \mathrm{~mL})$ was stirred for $16 \mathrm{~h}$ at ambient temperature. The solution was diluted with brine $(15 \mathrm{~mL})$ and extracted with $\mathrm{CH}_{2} \mathrm{Cl}_{2}(3 \times 20 \mathrm{~mL})$. The organic layers were dried $\left(\mathrm{MgSO}_{4}\right)$, filtered and the solvent was evaporated in vacuo. The residue was washed with THF $(20 \mathrm{~mL})$ and dried to furnish acetamide 13 (64 mg, $0.17 \mathrm{mmol}, 57 \%)$ as a colorless solid, mp. $273-275^{\circ} \mathrm{C} .{ }^{1} \mathrm{H}$ NMR (500 MHz, DMSO-d $)$ : 
$\delta=1.80(\mathrm{~d}, J=13.0 \mathrm{~Hz}, 2 \mathrm{H}), 2.04(\mathrm{~s}, 3 \mathrm{H}), 2.05(\mathrm{td}, J=13.2 \mathrm{~Hz}, J=2.6 \mathrm{~Hz}, 2 \mathrm{H}), 2.16$ (td, $J=13.4 \mathrm{~Hz}, J=2.5 \mathrm{~Hz}, 2 \mathrm{H}$ ), $2.34(\mathrm{~d}, J=12.4 \mathrm{~Hz}, 2 \mathrm{H}$ ), 2.70 (t, $J=5.3 \mathrm{~Hz}, 2 \mathrm{H}$ ), $3.95(\mathrm{t}, J=5.3 \mathrm{~Hz}, 2 \mathrm{H}), 6.98(\mathrm{t}, J=7.4 \mathrm{~Hz}, 1 \mathrm{H}), 7.07(\mathrm{t}, J=7.5 \mathrm{~Hz}, 1 \mathrm{H}), 7.20$ (t, J = 7.2 $\mathrm{Hz}, 1 \mathrm{H}), 7.33(\mathrm{t}, J=7.7 \mathrm{~Hz}, 2 \mathrm{H}), 7.36-7.43(\mathrm{~m}, 4 \mathrm{H}), 7.85(\mathrm{~s}, 1 \mathrm{H}), 10.88(\mathrm{~s}, 1 \mathrm{H}) \mathrm{ppm}$. ${ }^{13} \mathrm{C}\left\{{ }^{1} \mathrm{H}\right\}$ NMR (125 MHz, DMSO-d 6$): \delta=22.2\left(\mathrm{CH}_{2}\right), 24.1\left(\mathrm{CH}_{3}\right), 30.5\left(2 \mathrm{CH}_{2}\right), 30.9(2$ $\left.\mathrm{CH}_{2}\right), 56.7(\mathrm{C}), 59.0\left(\mathrm{CH}_{2}\right), 71.3(\mathrm{C}), 105.3(\mathrm{C}), 111.0(\mathrm{CH}), 117.7(\mathrm{CH}), 118.4(\mathrm{CH})$, $120.7(\mathrm{CH}), 124.9(2 \mathrm{CH}), 125.9(\mathrm{CH}), 126.5(\mathrm{C}), 127.9(2 \mathrm{CH}), 135.7(\mathrm{C}), 139.4(\mathrm{C})$, 148.3 (C), 169.4 (C) ppm. IR (ATR): 3410 (w), 3224 (w), 2923 (w), 2863 (w), 1651 (s), $1510(\mathrm{~m}), 1495(\mathrm{~m}), 1471(\mathrm{w}), 1444(\mathrm{~m}), 1428(\mathrm{~m}), 1367(\mathrm{w}), 1318(\mathrm{w}), 1290(\mathrm{~m}), 1271$ $(m), 1257(w), 1205(w), 1164(w), 1075(s), 1052(s), 1025(m), 968(w), 950(m), 928$ (w), $922(w), 770(m), 742(s), 731(m), 718(m), 701(s), 682(w), 655(w), 611(w)$, 537 (s) cm $\mathrm{cm}^{-1}$. MS (El, $70 \mathrm{eV}$ ): $\mathrm{m} / \mathrm{z}$ (\%) 374 (40) [M], 315 (10), 198 (28), 185 (100), 155 (12). HRMS (El, $70 \mathrm{eV}$ ) m/z: [M] calcd. for $\mathrm{C}_{24} \mathrm{H}_{26} \mathrm{~N}_{2} \mathrm{O}_{2}{ }^{+}$374.1989; found 374.1991. $\mathrm{C}_{24} \mathrm{H}_{26} \mathrm{~N}_{2} \mathrm{O}_{2}\left(374.48 \mathrm{~g} \mathrm{~mol}^{-1}\right)$.

\section{trans-4-[N-(4-lodobenzoyl)amino]-4-phenyl-4',9'-dihydro-3' H-spiro[cyclohexane-}

1,1'-pyrano[3,4-b]indole] (14). A solution of amine $12(0.10 \mathrm{~g}, 0.30 \mathrm{mmol})$, 4-iodobenzoic acid (149 mg, $0.601 \mathrm{mmol}), \mathrm{EDC} \cdot \mathrm{HCl}(115 \mathrm{mg}, 0.600 \mathrm{mmol})$ and NEt3 $(91$ $\mathrm{mg}, 0.90 \mathrm{mmol})$ in THF $(10 \mathrm{~mL})$ was stirred for $16 \mathrm{~h}$ at ambient temperature. The solution was diluted with hydrochloric acid $(1 \mathrm{~mol} / \mathrm{L}, 15 \mathrm{~mL})$ and extracted with $\mathrm{CH}_{2} \mathrm{Cl}_{2}$ (3 $x 20 \mathrm{~mL})$. The organic layers were dried $\left(\mathrm{MgSO}_{4}\right)$, filtered and the solvent was evaporated in vacuo to furnish the product $14(90 \mathrm{mg}, 0.16 \mathrm{mmol}, 53 \%)$ after chromatography $\left(\mathrm{SiO}_{2}\right.$, hexanes/MTBE 1:1, $\left.\mathrm{Rf}=0.43\right)$ as a colorless solid, mp. $209-211^{\circ} \mathrm{C} .{ }^{1} \mathrm{H}$ NMR $\left(500 \mathrm{MHz}, \mathrm{CDCl}_{3}\right): \delta=1.98-2.04(\mathrm{~m}, 4 \mathrm{H}), 2.46(\mathrm{dt}, J=14.6 \mathrm{~Hz}, J=7.3 \mathrm{~Hz}, 2 \mathrm{H}), 2.75-$ $2.82(\mathrm{~m}, 2 \mathrm{H}), 2.83(\mathrm{t}, J=5.4 \mathrm{~Hz}, 2 \mathrm{H}), 4.03(\mathrm{t}, J=5.4 \mathrm{~Hz}, 2 \mathrm{H}), 6.30(\mathrm{~s}, 1 \mathrm{H}), 7.11(\mathrm{t}, J=$ $7.4 \mathrm{~Hz}, 1 \mathrm{H}), 7.17(\mathrm{t}, J=7.6 \mathrm{~Hz}, 1 \mathrm{H}), 7.29(\mathrm{t}, J=7.3 \mathrm{~Hz}, 1 \mathrm{H}), 7.35-7.42(\mathrm{~m}, 3 \mathrm{H}), 7.51$ (d, $J=7.8 \mathrm{~Hz}, 1 \mathrm{H}), 7.55$ (dd, $J=8.3 \mathrm{~Hz}, J=2.3 \mathrm{~Hz}, 4 \mathrm{H}$ ), $7.83(\mathrm{~d}, J=8.3 \mathrm{~Hz}, 2 \mathrm{H}), 8.52$ (s, 1H) ppm. ${ }^{13} \mathrm{C}\left\{{ }^{1} \mathrm{H}\right\}$ NMR $\left(125 \mathrm{MHz}, \mathrm{CDCl}_{3}\right): \delta=22.7\left(\mathrm{CH}_{2}\right), 30.6\left(2 \mathrm{CH}_{2}\right), 32.5(2$ $\left.\mathrm{CH}_{2}\right), 58.6(\mathrm{C}), 60.3\left(\mathrm{CH}_{2}\right), 71.8(\mathrm{C}), 98.8(\mathrm{C}), 107.7(\mathrm{C}), 111.2(\mathrm{CH}), 118.4(\mathrm{CH}), 119.7$ $(\mathrm{CH}), 121.9(\mathrm{CH}), 125.5(2 \mathrm{CH}), 127.0(\mathrm{C}), 127.5(\mathrm{CH}), 128.6(2 \mathrm{CH}), 128.9(2 \mathrm{CH})$, 134.6 (C), 136.0 (C), 138.0 (C), $138.1(2 \mathrm{CH}), 145.4$ (C), 166.3 (C) ppm. IR (ATR): $3294(w), 3054(w), 2924(w), 1628(m), 1584(m), 1494(m), 1475(s), 1445(m), 1315$ (w), $1298(m), 1267(m), 1205(w), 1162(w), 1110(w), 1074(s), 1051(m), 1028(w)$, $1005(\mathrm{~s}), 985(\mathrm{w}), 895(\mathrm{w}), 860(\mathrm{w}), 837(\mathrm{~m}), 738(\mathrm{~s}), 700(\mathrm{~s}), 678(\mathrm{w}), 557(\mathrm{~m}), 540$ 
(w), 522 (w) cm-1. MS (El, $70 \mathrm{eV}): \mathrm{m} / \mathrm{z}$ (\%) 562 (38) [M]+, 315 (12), 231 (10), 198 (24), 185 (100), 155 (14). HRMS (El, $70 \mathrm{eV}$ ) m/z: [M] calcd. for $\mathrm{C}_{29} \mathrm{H}_{27} \mathrm{IN}_{2} \mathrm{O}_{2}{ }^{+} 562.1112$; found 562.1106. $\mathrm{C}_{29} \mathrm{H}_{27} \mathrm{IN}_{2} \mathrm{O}_{2}\left(562.45 \mathrm{~g} \mathrm{~mol}^{-1}\right)$.

\section{trans-4-\{N-[(S)-2-(tert-Butyloxycarbonylamino)-4,4-dimethylpentanoyl]amino\}-4-} phenyl-4',9'-dihydro-3' $H$-spiro[cyclohexane-1,1'-pyrano[3,4-b]indole] (15). A solution of amine $12(0.10 \mathrm{~g}, 0.30 \mathrm{mmol}), \mathrm{N}$-Boc-L-neopentylglycine (147 mg, 0.600 $\mathrm{mmol}), \mathrm{EDC} \cdot \mathrm{HCl}(115 \mathrm{mg}, 0.600 \mathrm{mmol})$ and NEt3 $(91 \mathrm{mg}, 0.90 \mathrm{mmol})$ in THF (10 mL) was stirred for $16 \mathrm{~h}$ at ambient temperature. The solution was diluted with hydrochloric acid $(1 \mathrm{~mol} / \mathrm{L}, 15 \mathrm{~mL})$ and extracted with $\mathrm{CH}_{2} \mathrm{Cl}_{2}(3 \times 20 \mathrm{~mL})$. The organic layers were dried $\left(\mathrm{MgSO}_{4}\right)$, filtered and the solvent was evaporated in vacuo to furnish the product $15(142 \mathrm{mg}, 0.254 \mathrm{mmol}, 83 \%)$ after chromatography $\left(\mathrm{SiO}_{2}\right.$, hexanes/MTBE 2:1, $\mathrm{R}_{\mathrm{f}}=$ 0.27 ) as a colorless solid, mp. $216-218^{\circ} \mathrm{C}$. [ $[\alpha]^{20}=+48.3^{\circ}\left(c=1.0 \mathrm{~g} / \mathrm{L}, \mathrm{CH}_{2} \mathrm{Cl}_{2}\right) .{ }^{1} \mathrm{H}$ NMR (500 MHz, CDCl $): \delta=1.04(\mathrm{~s}, 9 \mathrm{H}), 1.42(\mathrm{dd}, J=14.7 \mathrm{~Hz}, J=9.7 \mathrm{~Hz}, 1 \mathrm{H}), 1.52$ (s, $9 \mathrm{H}), 1.94-2.14(\mathrm{~m}, 6 \mathrm{H}), 2.27-2.38(\mathrm{~m}, 2 \mathrm{H}), 2.69-2.75(\mathrm{~m}, 1 \mathrm{H}), 2.80-2.87(\mathrm{~m}, 2 \mathrm{H})$, $4.02(\mathrm{t}, J=5.3 \mathrm{~Hz}, 2 \mathrm{H}$ ), 4.15 (ddd, $J=9.2 \mathrm{~Hz}, J=6.5 \mathrm{~Hz}, J=2.1 \mathrm{~Hz}, 1 \mathrm{H}$ ), $4.94(\mathrm{~s}, 1 \mathrm{H}$ ), $6.50(\mathrm{~s}, 1 \mathrm{H}), 7.11(\mathrm{t}, J=7.4 \mathrm{~Hz}, 1 \mathrm{H}), 7.16(\mathrm{t}, J=7.5 \mathrm{~Hz}, 1 \mathrm{H}), 7.25(\mathrm{t}, J=7.3 \mathrm{~Hz}, 1 \mathrm{H})$, 7.35 (t, $J=7.3 \mathrm{~Hz}, 2 \mathrm{H}), 7.41(\mathrm{~d}, J=8.0 \mathrm{~Hz}, 1 \mathrm{H}), 7.45(\mathrm{~d}, J=7.5 \mathrm{~Hz}, 2 \mathrm{H}), 7.52(\mathrm{~d}, J=$ $7.7 \mathrm{~Hz}, 1 \mathrm{H}), 9.35(\mathrm{~s}, 1 \mathrm{H}) \mathrm{ppm} .{ }^{13} \mathrm{C}\left\{{ }^{1} \mathrm{H}\right\} \mathrm{NMR}(125 \mathrm{MHz}, \mathrm{CDCl} 3): \delta=22.8\left(\mathrm{CH}_{2}\right), 28.6$ (3 $\left.\mathrm{CH}_{3}\right), 29.5\left(\mathrm{CH}_{2}\right), 29.9\left(3 \mathrm{CH}_{3}\right), 30.7(\mathrm{C}), 31.3\left(\mathrm{CH}_{2}\right), 31.6\left(\mathrm{CH}_{2}\right), 32.9\left(\mathrm{CH}_{2}\right), 45.5$ $\left(\mathrm{CH}_{2}\right), 54.4(\mathrm{CH}), 58.0(\mathrm{C}), 60.2\left(\mathrm{CH}_{2}\right), 71.5(\mathrm{C}), 81.4(\mathrm{C}), 106.8(\mathrm{C}), 111.2(\mathrm{CH}), 118.1$ $(\mathrm{CH}), 119.1(\mathrm{CH}), 121.5(\mathrm{CH}), 125.1(2 \mathrm{CH}), 126.9(\mathrm{CH}), 127.0(\mathrm{C}), 128.5(2 \mathrm{CH})$, 136.0 (C), 138.9 (C), 146.3 (C), 156.7 (C), 171.7 (C) ppm. IR (ATR): 3337 (w), 3284 (w), $2957(\mathrm{w}), 1685(\mathrm{~s}), 1658(\mathrm{~s}), 1527(\mathrm{~m}), 1511(\mathrm{~m}), 1495(\mathrm{~m}), 1472(\mathrm{w}), 1447(\mathrm{~m})$, $1368(\mathrm{~m}), 1300(\mathrm{~m}), 1280(\mathrm{~m}), 1267(\mathrm{~m}), 1250(\mathrm{~m}), 1234(\mathrm{w}), 1164(\mathrm{~m}), 1072(\mathrm{~m}), 1052$ (m), $905(\mathrm{~m}), 757$ (w), 740 (s), 727 (s), 695 (s), $674(\mathrm{~m}), 644(\mathrm{~m}), 634(\mathrm{w}), 621(\mathrm{w}), 607$ (m), $534(\mathrm{~m}) \mathrm{cm}^{-1}$. MS (El, $\left.70 \mathrm{eV}\right): \mathrm{m} / z$ (\%) 559 (40) [M]+, 459 (20), 315 (14), 198 (12), 185 (100), 155 (12), 134 (18), 100 (20), 57 (9). HRMS (El, $70 \mathrm{eV}$ ) m/z: [M] calcd. for $\mathrm{C}_{34} \mathrm{H}_{45} \mathrm{~N}_{3} \mathrm{O}_{4}+559.3405$; found 559.3414. $\mathrm{C}_{34} \mathrm{H}_{45} \mathrm{~N}_{3} \mathrm{O}_{4}\left(559.75 \mathrm{~g} \mathrm{~mol}^{-1}\right)$.

\section{trans-4-(4-Bromophenylsulfonylamino)-4-phenyl-4', 9'-dihydro-3' $\mathrm{H}$-spiro[cyclo-}

hexane-1,1'-pyrano[3,4-b]indole] (16). A solution of amine $12(0.10 \mathrm{~g}, 0.30 \mathrm{mmol})$, 4-bromobenzenesulfonyl chloride $(0.15 \mathrm{~g}, 0.60 \mathrm{mmol})$ and NEt3 $(61 \mathrm{mg}, 0.60 \mathrm{mmol})$ in THF (10 mL) was stirred $16 \mathrm{~h}$ at ambient temperature. The solution was diluted with 
brine $(15 \mathrm{~mL})$ and extracted with $\mathrm{CH}_{2} \mathrm{Cl}_{2}(3 \times 20 \mathrm{~mL})$. The organic layers were dried $\left(\mathrm{MgSO}_{4}\right)$, filtered and the solvent was evaporated in vacuo. The residue was chromatographed $\left(\mathrm{SiO}_{2}\right.$, hexanes/MTBE $\left.1: 1, \mathrm{R}_{\mathrm{f}}=0.14\right)$ to furnish sulfonamide $16(113 \mathrm{mg}$, $0.205 \mathrm{mmol}, 68 \%)$ as a colorless solid, mp. $238-240^{\circ} \mathrm{C} .{ }^{1} \mathrm{H} \mathrm{NMR}\left(500 \mathrm{MHz}, \mathrm{CDCl}_{3}\right): \delta$ $=2.06(\mathrm{~d}, J=13.5 \mathrm{~Hz}, 2 \mathrm{H}), 2.25(\mathrm{td}, J=13.6 \mathrm{~Hz}, J=3.1 \mathrm{~Hz}, 2 \mathrm{H}), 2.39(\mathrm{td}, J=14.2$ $\mathrm{Hz}, J=2.9 \mathrm{~Hz}, 2 \mathrm{H}), 2.49(\mathrm{~d}, J=12.9 \mathrm{~Hz}, 2 \mathrm{H}), 2.79$ (t, $J=5.4 \mathrm{~Hz}, 2 \mathrm{H}), 3.94$ (t, $J=5.4$ $\mathrm{Hz}, 2 \mathrm{H}), 5.99(\mathrm{~s}, 1 \mathrm{H}), 6.99-7.05(\mathrm{~m}, 2 \mathrm{H}), 7.06-7.20(\mathrm{~m}, 5 \mathrm{H}), 7.22-7.25(\mathrm{~m}, 2 \mathrm{H}), 7.28-$ $7.32(\mathrm{~m}, 2 \mathrm{H}), 7.39(\mathrm{~d}, J=8.1 \mathrm{~Hz}, 1 \mathrm{H}), 7.47(\mathrm{~d}, J=7.8 \mathrm{~Hz}, 1 \mathrm{H}), 8.50(\mathrm{~s}, 1 \mathrm{H}) \mathrm{ppm}$. ${ }^{13} \mathrm{C}\left\{{ }^{1} \mathrm{H}\right\}$ NMR $(125 \mathrm{MHz}, \mathrm{CDCl} 3): \delta=22.7\left(\mathrm{CH}_{2}\right), 31.1\left(2 \mathrm{CH}_{2}\right), 32.0\left(2 \mathrm{CH}_{2}\right), 59.2(\mathrm{C})$, $60.1\left(\mathrm{CH}_{2}\right), 71.4(\mathrm{C}), 107.3(\mathrm{C}), 111.4(\mathrm{CH}), 118.2(\mathrm{CH}), 119.6(\mathrm{CH}), 121.9(\mathrm{CH}), 126.4$ (2 CH), $126.95(\mathrm{C}), 127.04(\mathrm{C}), 127.3(\mathrm{CH}), 128.1(2 \mathrm{CH}), 128.5(2 \mathrm{CH}), 131.8(2 \mathrm{CH})$, 136.2 (C), 138.3 (C), 140.3 (C), 142.2 (C) ppm. IR (ATR): 3382 (w), 3250 (w), 2954 (w), $2923(w), 2859(w), 1574(m), 1472(w), 1414(w), 1389(w), 1330(m), 1300(w)$, $1210(\mathrm{w}), 1156(\mathrm{~s}), 1076(\mathrm{~s}), 1012(\mathrm{~m}), 983(\mathrm{~m}), 926(\mathrm{w}), 874(\mathrm{w}), 820(\mathrm{~m}), 763(\mathrm{~m})$, 739 (s), 699 (s), $609(\mathrm{~m}), 590(\mathrm{~m}), 543(\mathrm{~s}) \mathrm{cm}^{-1}$. MS (El, $\left.70 \mathrm{eV}\right): \mathrm{m} / \mathrm{z}(\%) 550(8)[\mathrm{M}]^{+}$, 303 (10), 286 (12), 198 (40), 185 (100), 155 (22), 104 (10). HRMS (El, 70 eV) m/z: [M] ${ }^{+}$ calcd. for $\mathrm{C}_{28} \mathrm{H}_{27} \mathrm{BrN}_{2} \mathrm{O}_{3} \mathrm{~S}^{+} 550.0920$; found 550.0918. $\mathrm{C}_{28} \mathrm{H}_{27} \mathrm{BrN}_{2} \mathrm{O}_{3} \mathrm{~S}(551.50 \mathrm{~g}$ $\left.\mathrm{mol}^{-1}\right)$.

\section{trans-4-\{N-[2-(Trifluoromethyl)phenylcarbamoyl]amino\}-4-phenyl-4',9'-dihydro-}

3'H-spiro[cyclohexane-1,1'-pyrano[3,4-b]indole] (17). A solution of amine 12 (0.10 $\mathrm{g}, 0.30 \mathrm{mmol}$ ) and 2-(trifluoromethyl)phenyl isocyanate (113 $\mathrm{mg}, 0.604 \mathrm{mmol})$ in THF $(10 \mathrm{~mL})$ was stirred for $16 \mathrm{~h}$ at $75^{\circ} \mathrm{C}$ in a tightly closed reaction vial. All volatile materials were removed under reduced pressure. The residue was chromatographed $\left(\mathrm{SiO}_{2}\right.$, hexanes/MTBE 2:1, $\left.R_{f}=0.10\right)$ to yield the product $17(103 \mathrm{mg}, 0.198 \mathrm{mmol}, 66 \%)$ as a

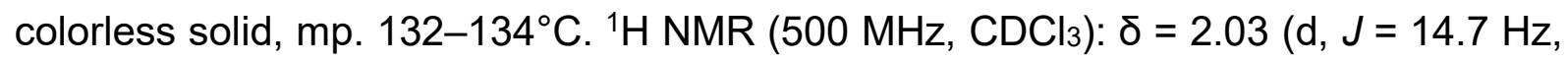
$2 \mathrm{H}$ ), 2.14-2.27 (m, 4H), 2.41 (td, $J=13.4 \mathrm{~Hz}, J=3.1 \mathrm{~Hz}, 2 \mathrm{H}$ ), $2.83(\mathrm{t}, J=5.4 \mathrm{~Hz}, 2 \mathrm{H}$ ), $4.01(\mathrm{t}, J=5.4 \mathrm{~Hz}, 2 \mathrm{H}), 5.72(\mathrm{~s}, 1 \mathrm{H}), 6.42(\mathrm{~s}, 1 \mathrm{H}), 7.05-7.16(\mathrm{~m}, 3 \mathrm{H}), 7.24(\mathrm{~d}, J=7.8$ $\mathrm{Hz}, 1 \mathrm{H}), 7.32(\mathrm{t}, J=7.4 \mathrm{~Hz}, 1 \mathrm{H}), 7.36(\mathrm{t}, J=8.2 \mathrm{~Hz}, 1 \mathrm{H}), 7.42(\mathrm{t}, J=7.7 \mathrm{~Hz}, 2 \mathrm{H}), 7.49$ (t, $J=6.5 \mathrm{~Hz}, 2 \mathrm{H}), 7.60(\mathrm{~d}, J=7.4 \mathrm{~Hz}, 2 \mathrm{H}), 7.88(\mathrm{~d}, J=8.3 \mathrm{~Hz}, 1 \mathrm{H}), 8.76(\mathrm{~s}, 1 \mathrm{H}) \mathrm{ppm}$. ${ }^{13} \mathrm{C}\left\{{ }^{1} \mathrm{H}\right\}$ NMR $(125 \mathrm{MHz}, \mathrm{CDCl} 3): \delta=22.7\left(\mathrm{CH}_{2}\right), 31.4\left(2 \mathrm{CH}_{2}\right), 32.0\left(2 \mathrm{CH}_{2}\right), 57.3(\mathrm{C})$, $60.2\left(\mathrm{CH}_{2}\right), 71.4(\mathrm{C}), 107.3(\mathrm{C}), 111.2(\mathrm{CH}), 118.2(\mathrm{CH}), 119.5(\mathrm{CH}), 121.1(\mathrm{q}, J=30$ $\mathrm{Hz}, \mathrm{C}), 121.8(\mathrm{CH}), 123.9(\mathrm{q}, J=273 \mathrm{~Hz}, \mathrm{C}), 124.2(\mathrm{CH}), 125.5(\mathrm{CH}), 125.7(2 \mathrm{CH})$, $126.2(\mathrm{q}, \mathrm{J}=5 \mathrm{~Hz}, \mathrm{CH}), 127.0(\mathrm{C}), 128.0(\mathrm{CH}), 129.3(2 \mathrm{CH}), 132.7(\mathrm{CH}), 135.9(\mathrm{C})$, 
136.0 (C), $138.3(\mathrm{C}), 145.3(\mathrm{C}), 155.8$ (C) ppm. ${ }^{19} \mathrm{~F}\left\{{ }^{1} \mathrm{H}\right\} \mathrm{NMR}\left(470 \mathrm{MHz}, \mathrm{CDCl}_{3}\right): \delta=-$ 61.09 (s) ppm. IR (ATR): 3328 (w), 2924 (w), 2851 (w), 1651 (m), $1590(w), 1494(w)$, $1448(\mathrm{~m}), 1318(\mathrm{~s}), 1297(\mathrm{~m}), 1267(\mathrm{~m}), 1232(\mathrm{~m}), 1204(\mathrm{w}), 1164(\mathrm{~m}), 1108(\mathrm{~s}), 1074$ (m), $1052(\mathrm{~m}), 1034(\mathrm{~m}), 957(\mathrm{w}), 907(\mathrm{w}), 757(\mathrm{~s}), 741(\mathrm{~s}), 697(\mathrm{~m}), 675(\mathrm{w}), 652(\mathrm{w})$, $597(w), 537$ (w), $521(w) \mathrm{cm}^{-1}$. MS (El, $\left.70 \mathrm{eV}\right): \mathrm{m} / \mathrm{z}$ (\%) 519 (24) [M] $]^{+}, 315$ (10), 198 (14), 185 (100), 155 (12), 134 (8). HRMS (El, $70 \mathrm{eV}$ ) m/z: [M] calcd. for $\mathrm{C}_{30} \mathrm{H}_{28} \mathrm{~F}_{3} \mathrm{~N}_{3} \mathrm{O}_{2}{ }^{+}$ 519.2128; found 519.2132. $\mathrm{C}_{30} \mathrm{H}_{28} \mathrm{~F}_{3} \mathrm{~N}_{3} \mathrm{O}_{2}\left(519.57 \mathrm{~g} \mathrm{~mol}^{-1}\right)$.

\section{trans-4-(Benzylamino)-4-phenyl-4',9'-dihydro-3' $H$-spiro[cyclohexane-1,1'-pyra-}

no[3,4-b]indole] (18a). A solution of amine $12(0.10 \mathrm{~g}, 0.30 \mathrm{mmol})$, benzaldehyde (35 $\mathrm{mg}, 0.33 \mathrm{mmol}$ ), anhydrous zinc chloride $(20 \mathrm{mg}, 0.15 \mathrm{mmol})$ and $\mathrm{NaCNBH}_{3}(21 \mathrm{mg}$, $0.33 \mathrm{mmol}$ ) in THF ( $5 \mathrm{~mL}$ ) was stirred for $16 \mathrm{~h}$ at ambient temperature. The solution was diluted with brine $(15 \mathrm{~mL})$ and extracted with $\mathrm{CH}_{2} \mathrm{Cl}_{2}(3 \times 20 \mathrm{~mL})$. The organic layers were dried $\left(\mathrm{MgSO}_{4}\right)$, filtered and the solvent was evaporated in vacuo. The residue was chromatographed $\left(\mathrm{SiO}_{2}\right.$, hexanes/MTBE $\left.3: 1, \mathrm{R}_{\mathrm{f}}=0.13\right)$ to furnish benzylamine $18 \mathrm{a}(81 \mathrm{mg}, 0.19 \mathrm{mmol}, 63 \%)$ as a colorless solid, mp. $229-231^{\circ} \mathrm{C} .{ }^{1} \mathrm{H}$ NMR (500 MHz, $\left.\mathrm{CDCl}_{3}\right): \delta=1.73(\mathrm{bs}, 1 \mathrm{H}), 1.91-2.00(\mathrm{~m}, 4 \mathrm{H}), 2.24-2.36(\mathrm{~m}, 4 \mathrm{H}), 2.82(\mathrm{t}, J$ $=5.4 \mathrm{~Hz}, 2 \mathrm{H}), 3.42(\mathrm{~s}, 2 \mathrm{H}), 4.02(\mathrm{t}, J=5.4 \mathrm{~Hz}, 2 \mathrm{H}), 7.09-7.14(\mathrm{~m}, 1 \mathrm{H}), 7.15-7.19(\mathrm{~m}$, $1 \mathrm{H}), 7.26-7.32(\mathrm{~m}, 2 \mathrm{H}), 7.32-7.39(\mathrm{~m}, 5 \mathrm{H}), 7.39-7.44(\mathrm{~m}, 2 \mathrm{H}), 7.49-7.54(\mathrm{~m}, 3 \mathrm{H}), 7.97$ (bs, $1 \mathrm{H})$ ppm. ${ }^{13} \mathrm{C}\left\{{ }^{1} \mathrm{H}\right\} \mathrm{NMR}\left(125 \mathrm{MHz}, \mathrm{CDCl}_{3}\right): \delta=22.7\left(\mathrm{CH}_{2}\right), 30.9\left(2 \mathrm{CH}_{2}\right), 31.2(2$ $\left.\mathrm{CH}_{2}\right), 47.2\left(\mathrm{CH}_{2}\right), 56.8(\mathrm{C}), 59.9\left(\mathrm{CH}_{2}\right), 72.1(\mathrm{C}), 107.3(\mathrm{C}), 111.0(\mathrm{CH}), 118.3(\mathrm{CH})$, $119.6(\mathrm{CH}), 121.8(\mathrm{CH}), 125.7(2 \mathrm{CH}), 126.6(\mathrm{CH}), 127.18(\mathrm{CH}), 127.24(\mathrm{C}), 128.4(2$ $\mathrm{CH}), 128.55(2 \mathrm{CH}), 128.64(2 \mathrm{CH}), 135.8(\mathrm{C}), 139.2(\mathrm{C}), 141.3(\mathrm{C}), 148.0(\mathrm{C}) \mathrm{ppm} . \mathrm{IR}$ (ATR): $3379(m), 3057$ (w), $3026(w), 2947(w), 2927(w), 2849(w), 1493(m), 1473$ $(m), 1432(m), 1373(w), 1316(w), 1302(m), 1256(w), 1223(w), 1189(w), 1163(w)$, $1077(\mathrm{~s}), 1057(\mathrm{~m}), 1042(\mathrm{~m}), 1029(\mathrm{~m}), 992(\mathrm{w}), 950(\mathrm{w}), 919(\mathrm{w}), 907(\mathrm{w}), 900(\mathrm{w})$, $869(\mathrm{w}), 830(\mathrm{w}), 753(\mathrm{~s}), 697(\mathrm{~s}), 676(\mathrm{~m}), 636(\mathrm{~m}), 610(\mathrm{~m}), 591(\mathrm{w}), 584(\mathrm{~m}), 546$ (m), $530(\mathrm{~m}) \mathrm{cm}^{-1}$. MS (El, $\left.70 \mathrm{eV}\right): \mathrm{m} / \mathrm{z}(\%) 422$ (12) [M] $]^{+}, 315$ (10), 185 (100), 155 (12), 91 (14), 44 (10). HRMS (El, $70 \mathrm{eV}$ ) m/z: [M] calcd. for $\mathrm{C}_{29} \mathrm{H}_{30} \mathrm{~N}_{2} \mathrm{O}^{+} 422.2353$; found 422.2345. $\mathrm{C}_{29} \mathrm{H}_{30} \mathrm{~N}_{2} \mathrm{O}\left(422.57 \mathrm{~g} \mathrm{~mol}^{-1}\right)$. 


\section{trans-4-(Isobutylamino)-4-phenyl-4',9'-dihydro-3' H-spiro[cyclohexane-1,1'-pyra-}

no[3,4-b]indole] (18b). A solution of amine $12(0.10 \mathrm{~g}, 0.30 \mathrm{mmol})$, isobutyraldehyde (24 mg, $0.33 \mathrm{mmol}$ ), anhydrous zinc chloride (20 mg, $0.15 \mathrm{mmol}$ ) and $\mathrm{NaCNBH}_{3}(21$ $\mathrm{mg}, 0.33 \mathrm{mmol})$ in THF $(5 \mathrm{~mL})$ was stirred $16 \mathrm{~h}$ at ambient temperature. The solution was diluted with brine $(15 \mathrm{~mL})$ and extracted with $\mathrm{CH}_{2} \mathrm{Cl}_{2}(3 \times 20 \mathrm{~mL})$. The organic layers were dried $\left(\mathrm{MgSO}_{4}\right)$, filtered and the solvent was evaporated in vacuo. The residue was chromatographed $\left(\mathrm{SiO}_{2}\right.$, hexanes/MTBE $\left.1: 1, \mathrm{Rf}_{\mathrm{f}}=0.19\right)$ to furnish amine $\mathbf{1 8 b}$ (40 mg, $0.10 \mathrm{mmol}, 33 \%)$ as a colorless solid, mp. 188-190 ${ }^{\circ} \mathrm{C} .{ }^{1} \mathrm{H} \mathrm{NMR}(500 \mathrm{MHz}$, $\mathrm{CDCl}_{3}$ ): $\delta=0.95$ (d, $J=6.6 \mathrm{~Hz}, 6 \mathrm{H}$ ), 1.00-2.00 (br. s, $1 \mathrm{H} ; \mathrm{NH}$ ), 1.67 (hept, $J=6.6 \mathrm{~Hz}$, $1 \mathrm{H}), 1.85$ (d, J = 12.8 Hz, 2H), 1.91 (d, J = 12.3 Hz, 2H), 2.05 (d, J = 6.6 Hz, 2H), 2.16$2.24(\mathrm{~m}, 2 \mathrm{H}), 2.29(\mathrm{td}, J=13.5 \mathrm{~Hz}, J=2.9 \mathrm{~Hz}, 2 \mathrm{H}), 2.81(\mathrm{t}, J=5.4 \mathrm{~Hz}, 2 \mathrm{H}), 4.01(\mathrm{t}, J$ $=5.4 \mathrm{~Hz}, 2 \mathrm{H}), 7.09-7.14(\mathrm{~m}, 1 \mathrm{H}), 7.15-7.20(\mathrm{~m}, 1 \mathrm{H}), 7.21-7.25(\mathrm{~m}, 1 \mathrm{H}), 7.33-7.43$ $(\mathrm{m}, 5 \mathrm{H}), 7.50(\mathrm{~d}, J=7.7 \mathrm{~Hz}, 1 \mathrm{H}), 7.76$ (br. s, $1 \mathrm{H})$ ppm. ${ }^{13} \mathrm{C}\left\{{ }^{1} \mathrm{H}\right\} \mathrm{NMR}(125 \mathrm{MHz}, \mathrm{CDCl})$ ): $\delta=21.2\left(2 \mathrm{CH}_{3}\right), 22.7\left(\mathrm{CH}_{2}\right), 29.5(\mathrm{CH}), 31.0\left(2 \mathrm{CH}_{2}\right), 31.2\left(2 \mathrm{CH}_{2}\right), 50.3\left(\mathrm{CH}_{2}\right), 56.0$ (C), $59.9\left(\mathrm{CH}_{2}\right), 72.2(\mathrm{C}), 107.3(\mathrm{C}), 111.0(\mathrm{CH}), 118.3(\mathrm{CH}), 119.6(\mathrm{CH}), 121.8(\mathrm{CH})$, $125.6(2 \mathrm{CH}), 126.3(\mathrm{CH}), 127.3(\mathrm{C}), 128.3(2 \mathrm{CH}), 135.8(\mathrm{C}), 139.4(\mathrm{C}), 148.5(\mathrm{C})$ ppm. IR (ATR): 3466 (w), 2954 (m), 2914 (m), 2863 (w), 2840 (w), 1466 (m), 1443 (m), 1372 (w), 1313 (w), 1292 (m), 1264 (w), $1213(w), 1159$ (w), 1077 (s), $1054(\mathrm{~m}), 1032$ (m), $989(w), 912(w), 764(m), 741(s), 714(m), 694(s), 674(m), 640(w), 579(w)$, $564(\mathrm{~m}), 544(\mathrm{w}), 530(\mathrm{~m}) \mathrm{cm}^{-1}$. MS (El, $\left.70 \mathrm{eV}\right): \mathrm{m} / \mathrm{z}(\%) 388(10)\left[\mathrm{M}^{+}, 315(8), 185\right.$ (100), 155 (10), 115 (4). HRMS (El, $70 \mathrm{eV}$ ) m/z: [M] calcd. for $\mathrm{C}_{26} \mathrm{H}_{32} \mathrm{~N}_{2} \mathrm{O}^{+} 388.2509$; found 388.2500. $\mathrm{C}_{26} \mathrm{H}_{32} \mathrm{~N}_{2} \mathrm{O}\left(388.56 \mathrm{~g} \mathrm{~mol}^{-1}\right)$. 


\section{Preparation of compound 5 - optimization}

Reagents and reaction conditions studies for the ester saponification/decarboxylation of compound $\mathbf{9}$.
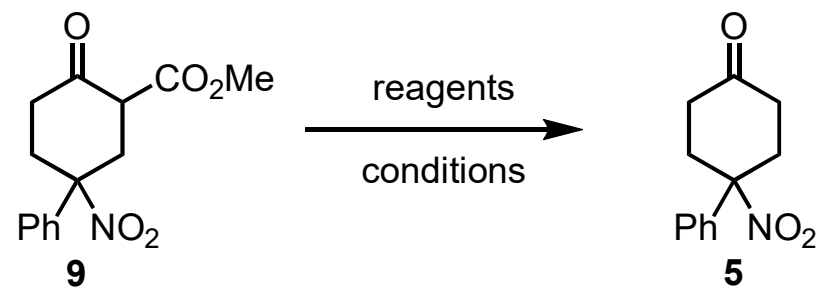

\begin{tabular}{|c|c|c|c|c|c|}
\hline Entry & Reagents & Solvent & $\mathrm{T} /{ }^{\circ} \mathrm{C}$ & $\mathrm{t} / \mathrm{h}$ & yield of 5 \\
\hline 1 & $\mathrm{KBr}$ & $\mathrm{DMSO} / \mathrm{H}_{2} \mathrm{O}$ & 110 & 16 & $17 \%$ \\
\hline 2 & $\mathrm{KBr}$ & $\mathrm{DMSO} / \mathrm{H}_{2} \mathrm{O}$ & 120 & 16 & $21 \%$ \\
\hline 3 & $\mathrm{NaCl}$ & $\mathrm{DMSO} / \mathrm{H}_{2} \mathrm{O}$ & 120 & 16 & $39 \%$ \\
\hline 4 & $\mathrm{NaCl}$ & $\mathrm{DMSO} / \mathrm{H}_{2} \mathrm{O}$ & 150 & 16 & $0 \%$ \\
\hline 5 & $\mathrm{NaCl}$ & $\mathrm{DMSO} / \mathrm{H}_{2} \mathrm{O}$ & 100 & 16 & $0 \%$ \\
\hline 6 & $\mathrm{NaCl}$ & $\mathrm{DMSO} / \mathrm{H}_{2} \mathrm{O}$ & 140 & 16 & $0 \%$ \\
\hline 7 & $\mathrm{NaCl}$ & $\mathrm{DMSO} / \mathrm{H}_{2} \mathrm{O}$ & 80 & 70 & $0 \%$ \\
\hline 8 & $\mathrm{NaCl}$ & DMF & 100 & 16 & $0 \%$ \\
\hline 9 & $\mathrm{NaCl}$ & DMF & 120 & 48 & $8 \%$ \\
\hline 10 & $\mathrm{LiCl}$ & $\mathrm{DMSO} / \mathrm{H}_{2} \mathrm{O}$ & 120 & 16 & $0 \%$ \\
\hline 11 & $\mathrm{LiCl}$ & DMA & 100 & 16 & $0 \%$ \\
\hline 12 & $\mathrm{~K}_{2} \mathrm{CO}_{3}$ & $\mathrm{MeOH}$ & 70 & 16 & $0 \%$ \\
\hline 13 & $\mathrm{~K}_{2} \mathrm{CO}_{3}$ & $\mathrm{DMSO} / \mathrm{H}_{2} \mathrm{O}$ & 120 & 16 & $0 \%$ \\
\hline 14 & $\mathrm{HCl}-\mathrm{H}_{2} \mathrm{O}(50 \%)$ & - & 100 & 16 & $0 \%$ \\
\hline 15 & $\mathrm{H}_{2} \mathrm{SO}_{4}$ (conc.) & - & 150 & 16 & $0 \%$ \\
\hline 16 & citric acid & $\mathrm{THF} / \mathrm{H}_{2} \mathrm{O}$ & 60 & 16 & $0 \%$ \\
\hline 17 & $\mathrm{NaOH}-\mathrm{H}_{2} \mathrm{O}(50 \%)$ & $\mathrm{EtOH}$ & 60 & 16 & $0 \%$ \\
\hline 18 & $\mathrm{NaOH}-\mathrm{H}_{2} \mathrm{O}(50 \%)$ & $\mathrm{EtOH}$ & 85 & 16 & $0 \%$ \\
\hline 19 & $\mathrm{NaOH}-\mathrm{H}_{2} \mathrm{O}(50 \%)$ & dioxane & 100 & 16 & $0 \%$ \\
\hline 20 & $\mathrm{NaOH}$ & $\mathrm{CH}_{2} \mathrm{Cl}_{2} / \mathrm{MeOH}$ & 25 & 16 & $0 \%$ \\
\hline 21 & $\mathrm{NaOH}-\mathrm{H}_{2} \mathrm{O}(2 \mathrm{~mol} / \mathrm{L})$ & THF & 85 & 16 & $0 \%$ \\
\hline 22 & $\mathrm{DABCO}$ & xylenes & 140 & 16 & $38 \%$ \\
\hline 23 & DBU & toluene & 140 & 16 & $0 \%$ \\
\hline 24 & $\mathrm{NEt}_{3}$ & toluene & 140 & 16 & $0 \%$ \\
\hline 25 & pyridine & - & 120 & 16 & $0 \%$ \\
\hline 26 & DMAP & xylenes $/ \mathrm{H}_{2} \mathrm{O}$ & 140 & 16 & $22 \%$ \\
\hline
\end{tabular}




\begin{tabular}{|c|c|c|c|c|c|}
\hline Entry & Reagents & Solvent & $\mathrm{T} /{ }^{\circ} \mathrm{C}$ & $\mathrm{t} / \mathrm{h}$ & yield of 5 \\
\hline 27 & $\mathrm{H}_{3} \mathrm{BO}_{3}$ & - & 180 & 16 & $0 \%$ \\
\hline 28 & $\mathrm{ZnCl}_{2} / \mathrm{HCl}$ & $\mathrm{EtOH} / \mathrm{H}_{2} \mathrm{O}$ & 80 & 16 & $25 \%$ \\
\hline 29 & - & - & 120 & 16 & $0 \%$ \\
\hline 30 & - & - & 150 & 16 & $30 \%$ \\
\hline 31 & - & - & 200 & 16 & $0 \%$ \\
\hline 32 & - & - & 150 & 2 & $33 \%$ \\
\hline 33 & - & TEG & 120 & 16 & $15 \%$ \\
\hline 34 & - & TEG & 150 & 16 & $14 \%$ \\
\hline 35 & $\mathrm{MgCl}_{2}$ & $\mathrm{H}_{2} \mathrm{O}$ & 100 & 16 & $5 \%$ \\
\hline 36 & $\mathrm{MgCl}_{2}$ & $\mathrm{DMSO} / \mathrm{H}_{2} \mathrm{O}$ & 130 & 16 & $23 \%$ \\
\hline 37 & - & triglyme & 150 & 16 & $0 \%$ \\
\hline 38 & DOWEX50WX8 & $\mathrm{MeOH} / \mathrm{H}_{2} \mathrm{O}$ & 80 & 16 & $15 \%$ \\
\hline 39 & DOWEX50Wx8 & EtOAc & 80 & 16 & $0 \%$ \\
\hline 40 & $\mathrm{LiBr}$ & DMPU & 80 & 16 & $32 \%$ \\
\hline 41 & $\mathrm{LiBr}, \mathrm{NEt}_{3}$ & $\mathrm{THF} / \mathrm{H}_{2} \mathrm{O}$ & 25 & 16 & $0 \%$ \\
\hline 42 & $\mathrm{LiBr}$ & DMPU & 80 & 16 & $38 \%$ \\
\hline 43 & $\mathrm{NaCl}$ & DMPU & 80 & 16 & $0 \%$ \\
\hline 44 & $\mathrm{NaBr}$ & DMPU & 80 & 16 & $24 \%$ \\
\hline 45 & $\mathrm{LiCl}$ & DMPU & 80 & 16 & $28 \%$ \\
\hline 46 & LiBr, TFA & DMPU & 80 & 16 & $16 \%$ \\
\hline 47 & $\mathrm{LiBr}$ & $\mathrm{DMPU} / \mathrm{H}_{2} \mathrm{O}$ & 85 & 16 & $0 \%$ \\
\hline 48 & $\mathrm{LiBr}$ & DMPU & 85 & 16 & $28 \%$ \\
\hline 49 & $\mathrm{LiBr}, \mathrm{CSA}$ & DMPU & 85 & 16 & $34 \%$ \\
\hline 50 & LiBr, CSA & DMPU & 100 & 16 & $61 \%$ \\
\hline 51 & $\mathrm{LiBr}, \mathrm{CSA}$ & DMPU & 120 & 16 & $0 \%$ \\
\hline
\end{tabular}




\section{Crystal structure of compound cis-11a}

Crystals of compound 11a suitable for X-ray analysis were obtained within three days from a saturated solution in DMSO- $\mathrm{d}_{6}$ at $25^{\circ} \mathrm{C}$. Single crystal $\mathrm{X}$-ray data were measured on a Bruker AXS D8 Venture diffractometer (multilayer optics, Mo-Ka radiation with $\lambda=0.71073 \AA$, Kappa 4-circle goniometer, Photon III C14 CPAD detector). An empirical absorption corrections using equivalent reflections was performed with the program SADABS. ${ }^{511}$ The structure was solved with the program SHELXS ${ }^{S 12}$ and refined with SHELXL ${ }^{S 13}$ using the OLEX2S14 GUI. All non-hydrogen atoms were refined using anisotropic atomic displacement parameters, hydrogen atoms bonded to carbon were located in the difference Fourier map and placed on idealized geometric positions with idealized atomic displacement parameters using the riding model, the hydrogen atom bonded to nitrogen and involved in the hydrogen bond was refined freely. The crystallographic data can be obtained free of charge from https://www.ccdc.cam.ac.uk/structures/ quoting the CCDC number 2012244. 
Table S1. Crystal data and structure refinement for compound 11a.

Empirical formula

Formula weight

Temperature

Wavelength

Crystal system

Space group

Unit cell dimensions

Volume

Z

Density (calculated)

Absorption coefficient

$\mathrm{F}(000)$

Crystal size

Theta range for data collection

Index ranges

Reflections collected

Independent reflections

Observed reflections [l > 2(I)]

Completeness to $\theta=36.317^{\circ}$

Absorption correction

Max. and min. transmission

Refinement method

Data / restraints / parameters

Goodness-of-fit on $\mathrm{F}^{2}$

Final $R$ indices [l $>2 \sigma(I)]$

$\mathrm{R}$ indices (all data)

Extinction coefficient

Largest diff. peak and hole
$\mathrm{C}_{24} \mathrm{H}_{28} \mathrm{~N}_{2} \mathrm{O}_{4} \mathrm{~S}$

$440.54 \mathrm{~g} \mathrm{~mol}^{-1}$

$100(2) \mathrm{K}$

$0.71073 \AA$

monoclinic

$\mathrm{P} 21 / \mathrm{n}$

$a=9.7400(4) \AA \quad \alpha=90^{\circ}$.

$b=13.2243(5) \AA \quad \beta=99.5302(17)^{\circ}$.

$c=16.9958(7) \AA \quad Y=90^{\circ}$.

2158.92(15) $\AA^{3}$

4

$1.355 \mathrm{~g} / \mathrm{mL}$

$0.184 \mathrm{~mm}^{-1}$

936

$0.120 \times 0.090 \times 0.080 \mathrm{~mm}^{3}$

1.962 to $36.317^{\circ}$

$-16 \leq \mathrm{h} \leq 16,-22 \leq \mathrm{k} \leq 22,-28 \leq \mathrm{I} \leq 28$

176526

$10474[R($ int $)=0.0440]$

9213

$100.0 \%$

Semi-empirical from equivalents

1.0000 and 0.9291

Full-matrix least-squares on $\mathrm{F}^{2}$

10474 / 0 / 286

1.091

$\mathrm{R} 1=0.0344, \mathrm{wR} 2=0.0912$

$\mathrm{R} 1=0.0406, \mathrm{wR} 2=0.0948$

$\mathrm{n} / \mathrm{a}$

0.554 and $-0.429 e^{-} \times \AA^{-3}$ 
Figure S1. ORTEP-representation (ellipsoids at the $50 \%$ probability level) of the structure of compound cis-11a in the solid state (as the solvate with DMSO-d6); hydrogen atoms and DMSO-d 6 are omitted for clarity.

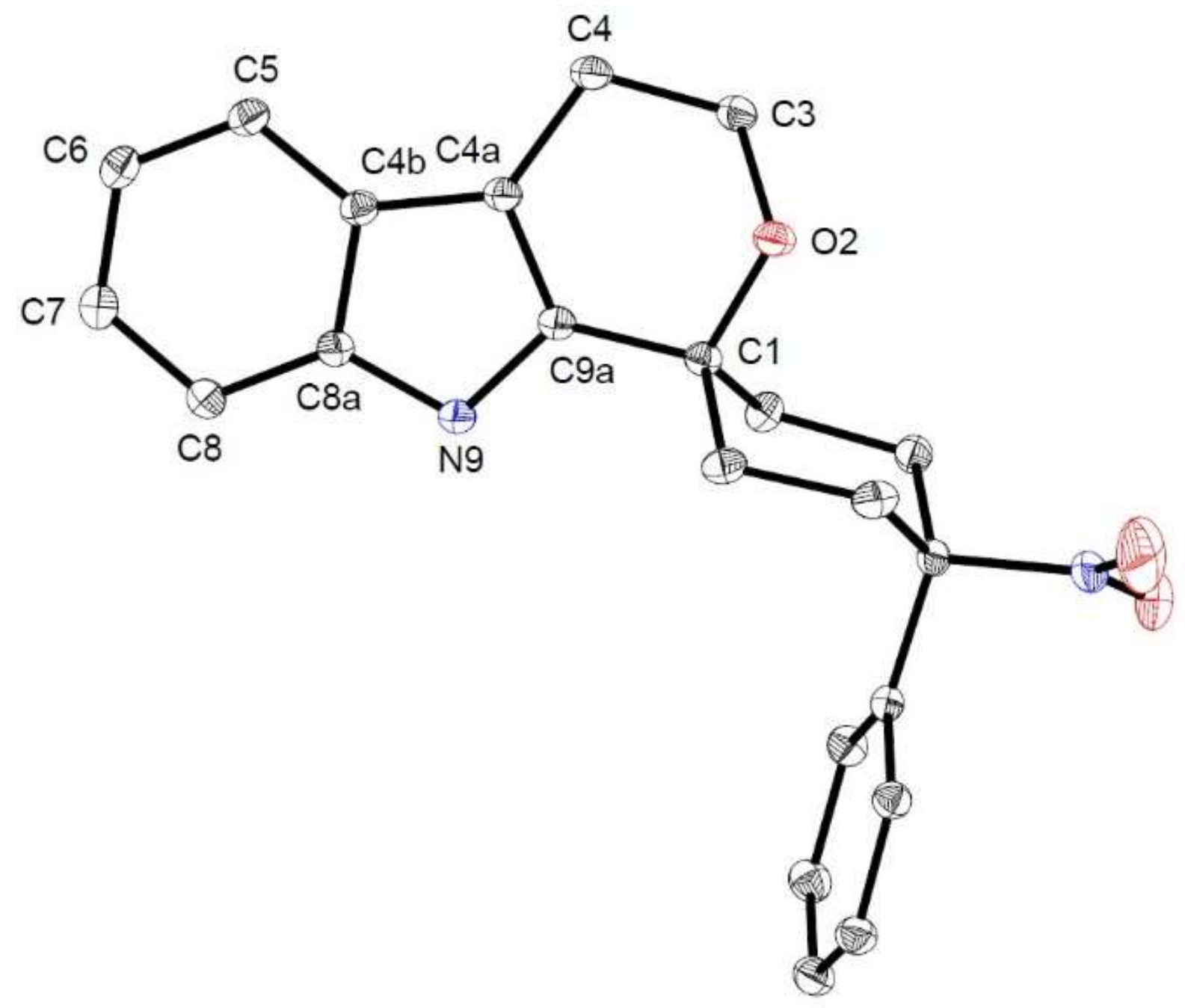




\section{References}

(S1) Kudyba, I.; Jozwik, J.; Romanski, J.; Raczko, J.; Jurczak, J. Tetrahedron Asymm. 2005, 16, 2257-2262.

(S2) Szostak, M.; Spain, M.; Eberhart, A. J.; Procter, D. J. J. Am. Chem. Soc. 2014, 136, 2268-2271.

(S3) Alpers, D.; Gallhof, M.; Witt, J.; Hoffmann, F.; Brasholz, M. Angew. Chem. Int. Ed. 2017, 56, 1402-1406; Angew. Chem. 2017, 129, 1423-1427.

(S4) Go, M.-L.; Leow, J. L.; Gorla, S. K.; Schüller, A. P.; Wang, M.; Casey, P. J. J. Med. Chem. 2010, 53, 6838-6850.

(S5) Christoffers, J.; Schuster, K. Chirality 2003, 15, 777-782.

(S6) Cai, S.; Zhang, S.; Zhao, Y.; Wang, D. Z. Org. Lett. 2013, 15, 2660-2663.

(S7) Campos, K. R.; Woo, J. C. S.; Lee, S.; Tillyer, R. D. Org. Lett. 2004, 6, 79-82.

(S8) González-Calderón, D.; Benitez-Puebla, L. J.; Gonzalez-Gonzalez, C. A.; Garcia-Eleno, M. A.; Fuentes-Benitez, A.; Cuevas-Yañez, E.; Corona-Becerril, D.; González-Romero, C. Synth. Commun. 2014, 44, 1258-1265.

(S9) Mewshaw, R. E.; Zhou, D.; Zhou, P.; Shi, X.; Hornby, G.; Spangler, T.; Scerni, R.; Smith, D.; Schechter, L. E.; Andree, T. H. J. Med. Chem. 2004, 47, 38233842.

(S10) Schunk, S.; Linz, K.; Hinze, C.; Frormann, S.; Oberbörsch, S.; Sundermann, B.; Zemolka, S.; Englberger, W.; Germann, T.; Christoph, T.; Kögel, B.-Y.; Schröder, W.; Harlfinger, S.; Saunders, D.; Kless, A.; Schick, H.; Sonnenschein, H. ACS Med. Chem. Lett. 2014, 5, 857-862.

(S11) Krause, L.; Herbst-Irmer, R.; Sheldrick, G. M.; Stalke, D. J. Appl. Cryst. 2015, 48, 3-10.

(S12) Sheldrick, G. M. Acta Cryst. 2008, A64, 112-122.

(S13) Sheldrick, G. M. Acta Cryst. 2015, C71, 3-8.

(S14) Dolomanov, O. V.; Bourhis, L. J.; Gildea, R. J.; Howard, J. A. K.; Puschmann, H. J. Appl. Cryst. 2009, 42, 339-341. 
6. ${ }^{1} \mathrm{H},{ }^{13} \mathrm{C}\left\{{ }^{1} \mathrm{H}\right\}$ and ${ }^{19} \mathrm{~F}\left\{{ }^{1} \mathrm{H}\right\}$ NMR spectra

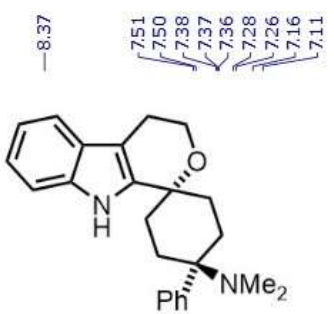

Compound 1a

${ }^{1} \mathrm{H}$ NMR $\left(500 \mathrm{MHz}, \mathrm{CDCl}_{3}\right)$
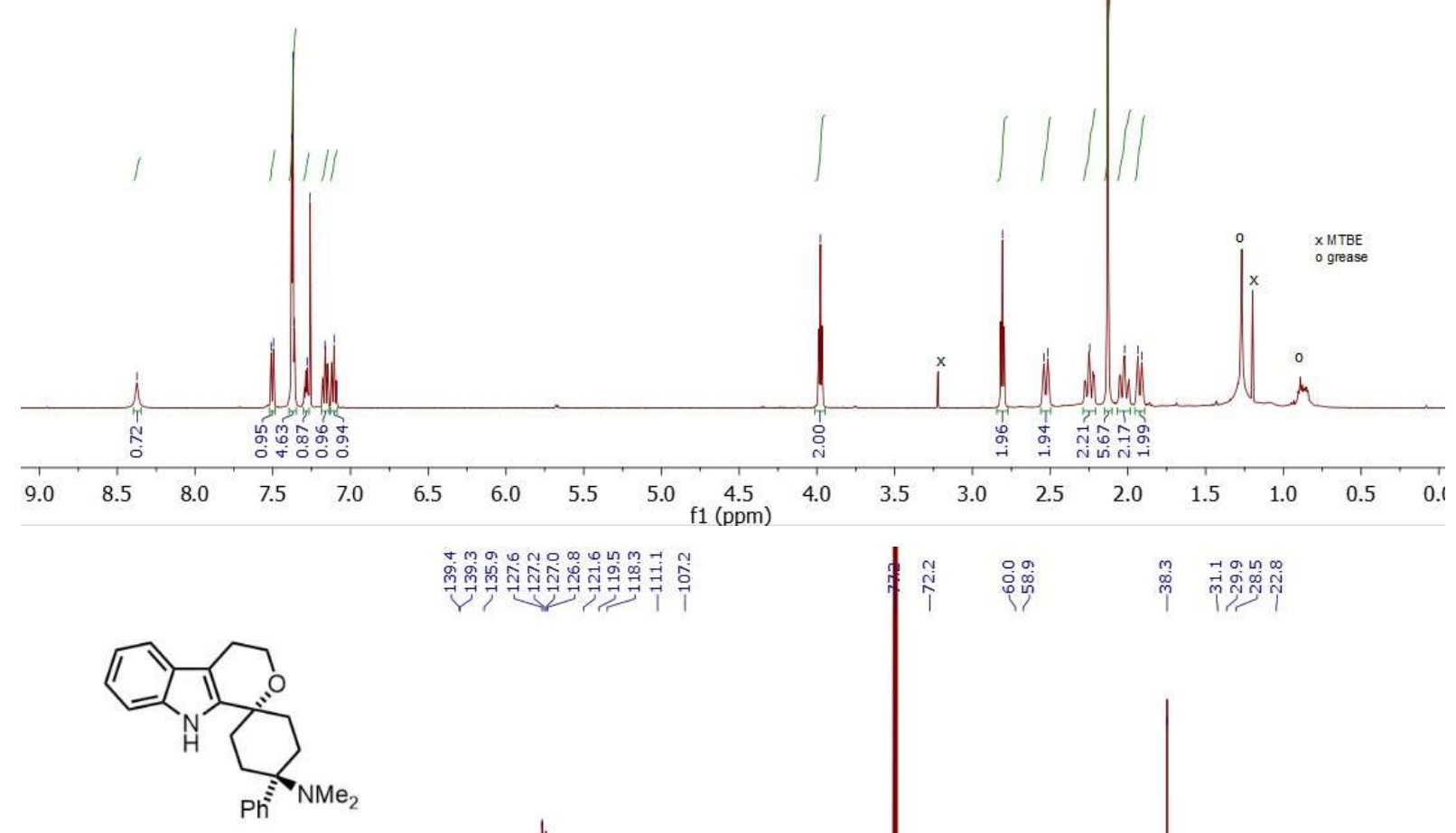

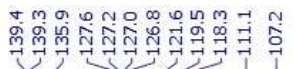

Compound 1a

${ }^{13} \mathrm{C}\left\{{ }^{1} \mathrm{H}\right\}$ NMR $\left(125 \mathrm{MHz}, \mathrm{CDCl}_{3}\right)$

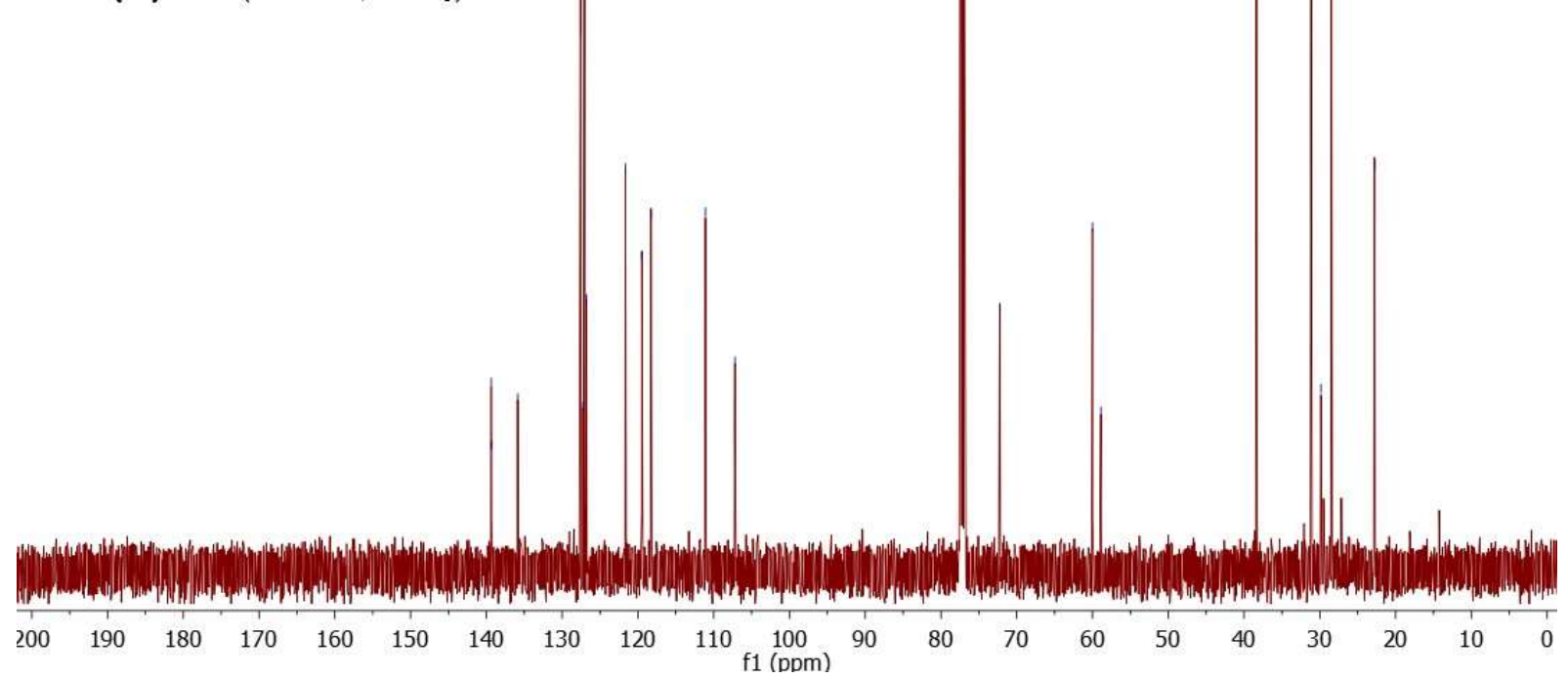




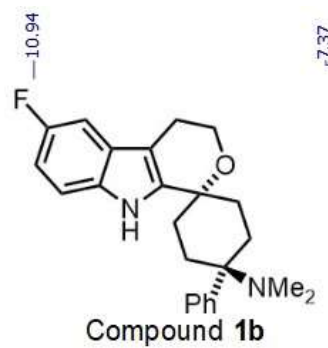

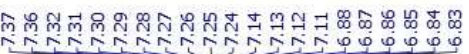

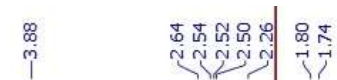

${ }^{1} \mathrm{H}$ NMR (500 MHz, DMSO)

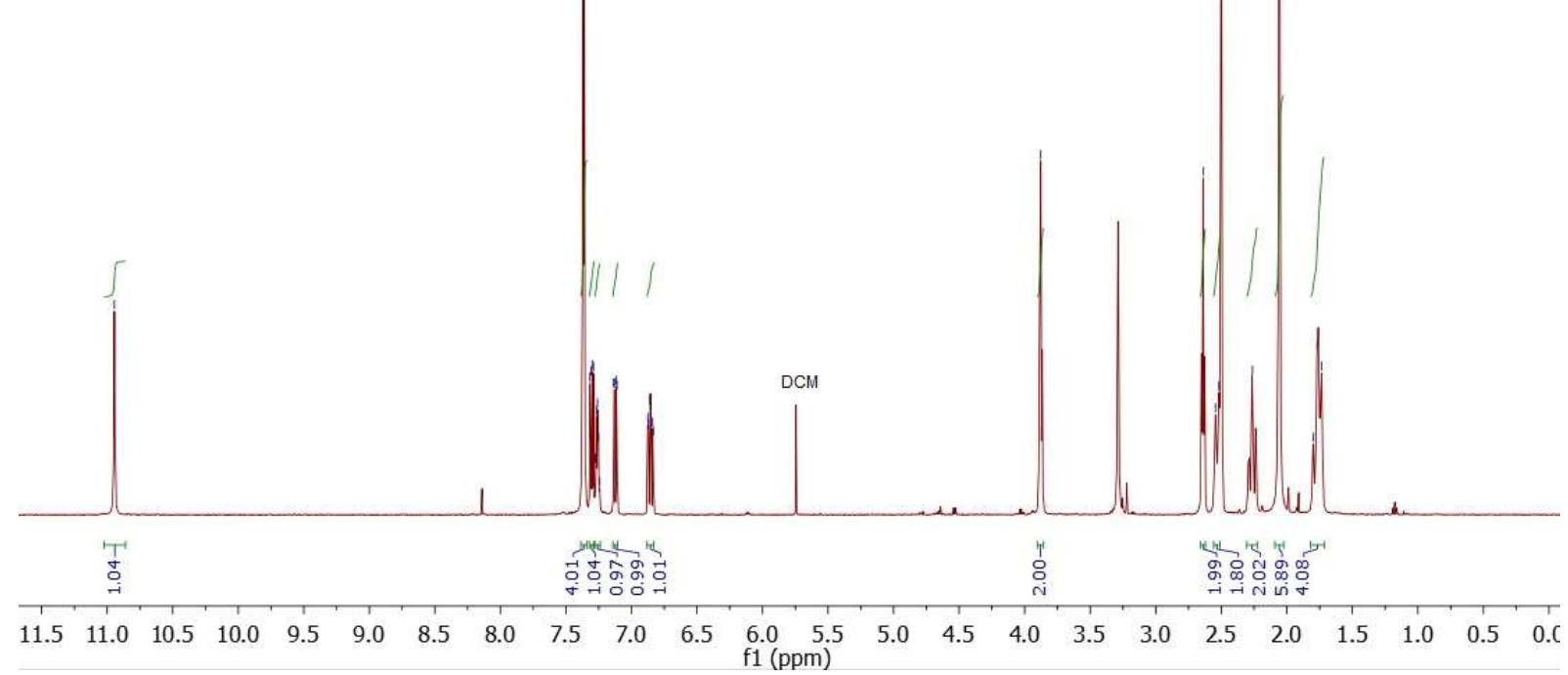

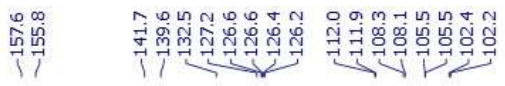

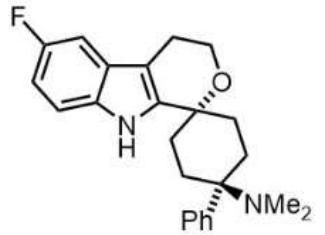

Compound 1b

${ }^{13} \mathrm{C}\left\{{ }^{1} \mathrm{H}\right\}$ NMR (125 MHz, DMSO)

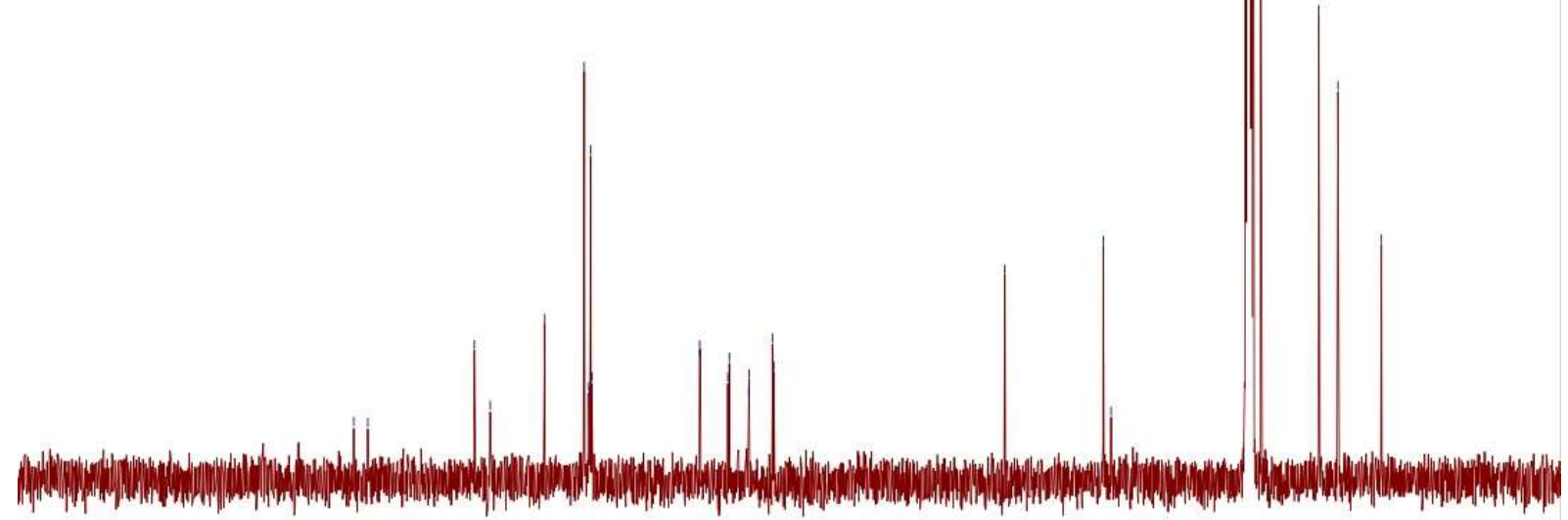

$\begin{array}{lllllllllllllllllllll}200 & 190 & 180 & 170 & 160 & 150 & 140 & 130 & 120 & 110 & 100 & 90 & 80 & 70 & 60 & 50 & 40 & 30 & 20 & 10 & 0\end{array}$ 


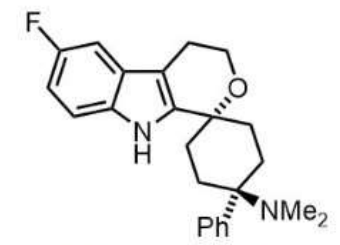

Compound 1b

${ }^{19} \mathrm{~F}\left\{{ }^{1} \mathrm{H}\right\} \mathrm{NMR}(470 \mathrm{MHz}, \mathrm{DMSO})$

$\begin{array}{llllllllllllll}30 & 20 & 10 & 0 & -10 & -20 & -30 & -40 & -50 & -60 & -70 & -80 & -90 & -100\end{array}-110-120-130-140-150-160-170-180-190-200-210-220-230-240-250$ f1 (ppm) 


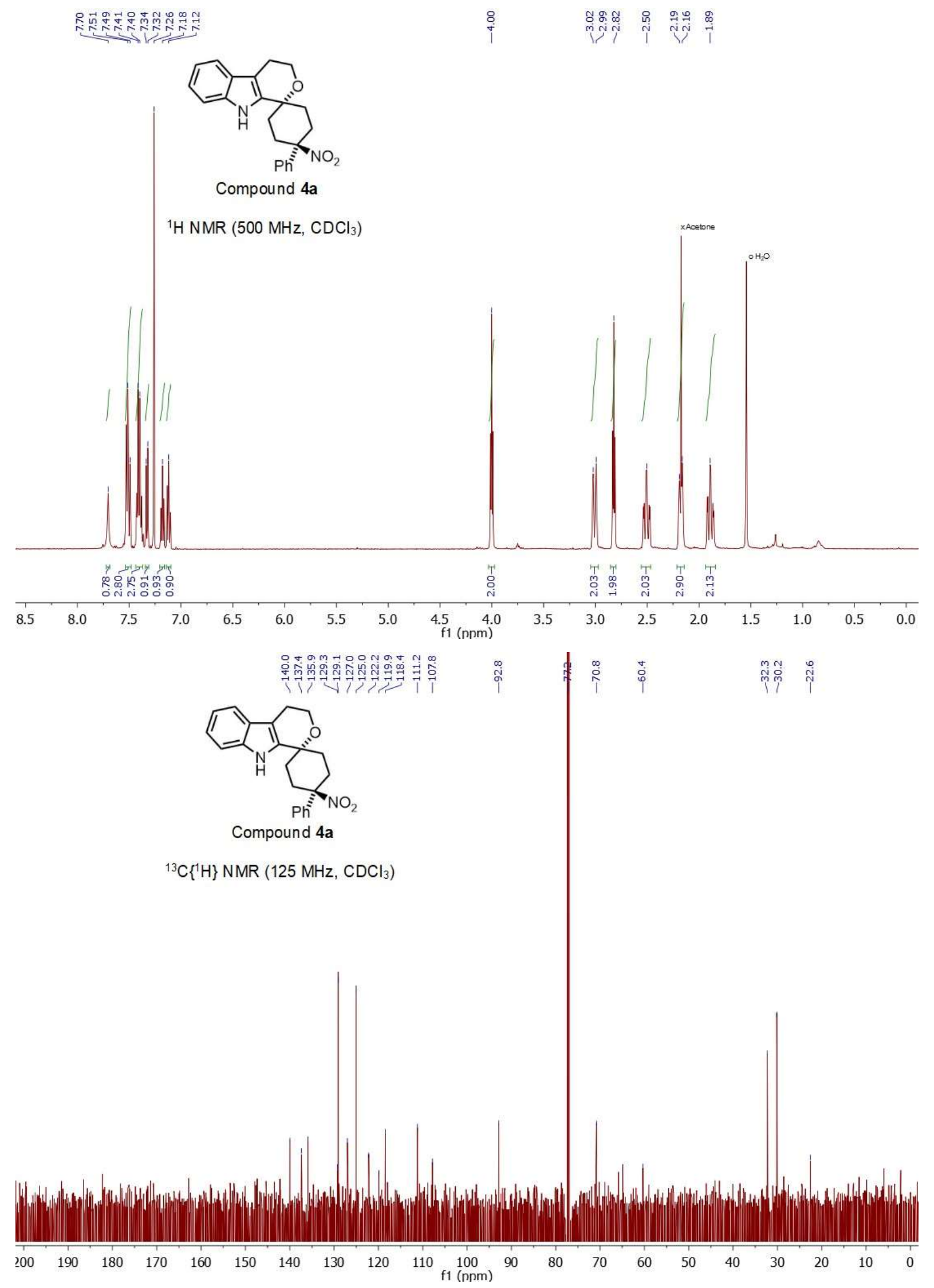


न<smiles>O=[N+]([O-])[C@]1(c2ccccc2)CC[C@]2(CC1)OCCc1c3cc(F)ccc3[nH]c12</smiles>

Compound $\mathbf{4 b}$

${ }^{1} \mathrm{H}$ NMR $(500 \mathrm{MHz}, \mathrm{DMSO})$
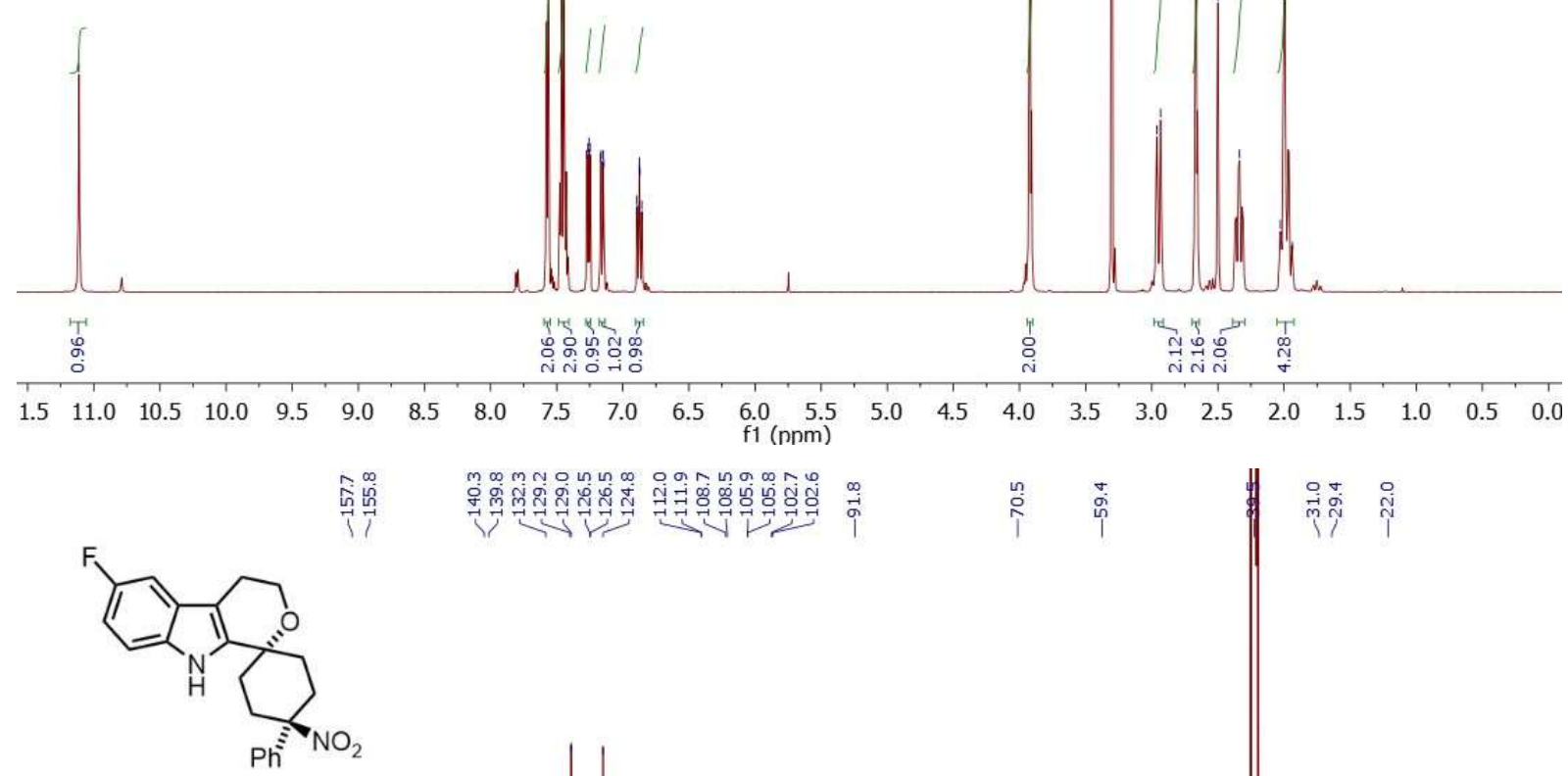

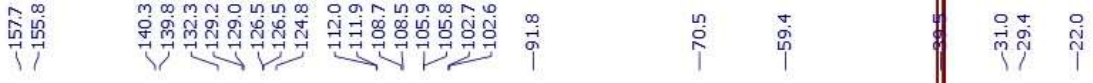

Compound $4 \mathrm{~b}$

${ }^{13} \mathrm{C}\left\{{ }^{1} \mathrm{H}\right\}$ NMR (125 MHz, DMSO)

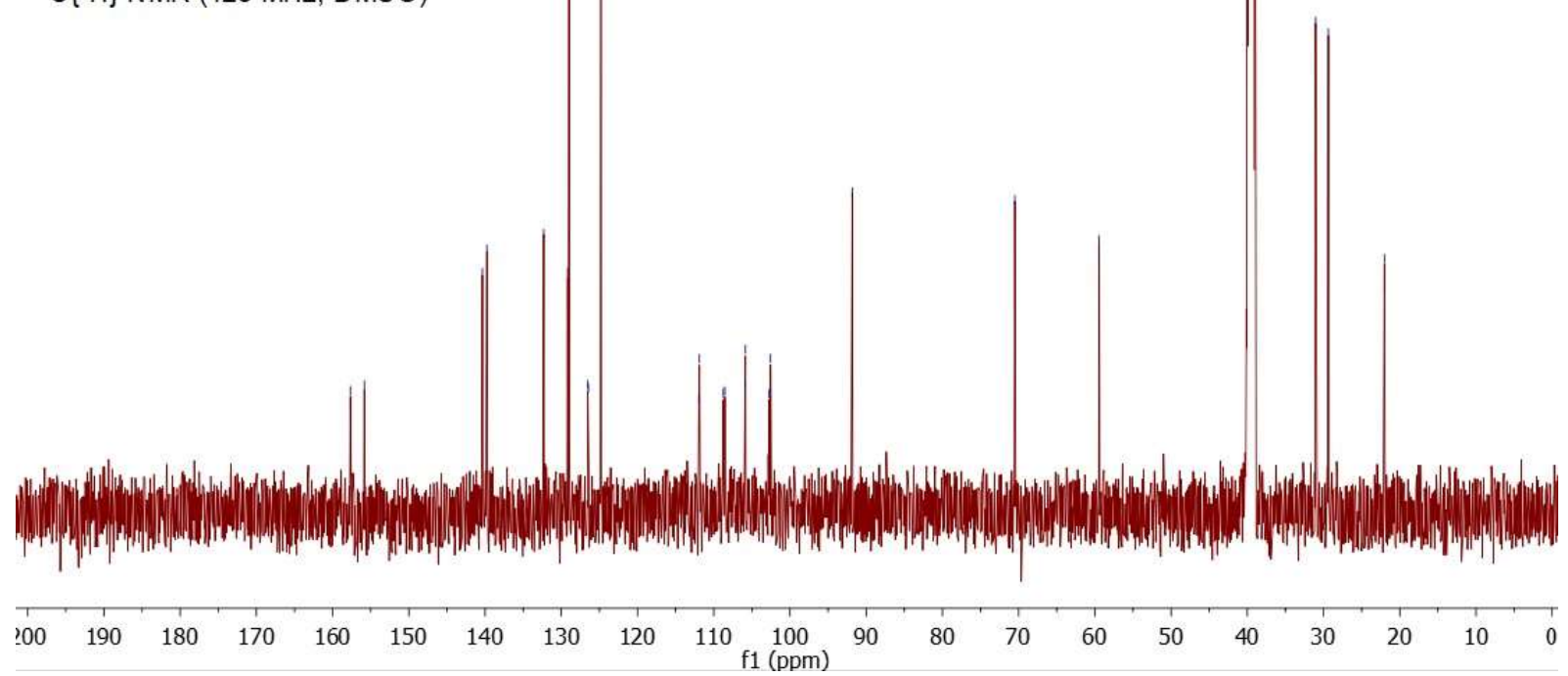




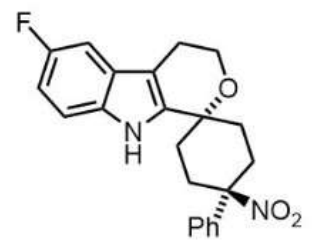

Compound $4 \mathrm{~b}$

${ }^{19} \mathrm{~F}\left\{{ }^{1} \mathrm{H}\right\} \mathrm{NMR}(470 \mathrm{MHz}, \mathrm{DMSO})$ $\begin{array}{llllllllllllll}30 & 20 & 10 & 0 & -10 & -20 & -30 & -40 & -50 & -60 & -70 & -80 & -90 & -100-110-120 \\ \mathrm{f} 1(\mathrm{ppm}) & -130-140-150-160-170-180-190-200-210-220-230-240-250\end{array}$ 
$\underset{\substack{1 \\ \hdashline}}{\stackrel{1}{1}}$<smiles>O=[N+]([O-])[C@]1(c2ccccc2)CC[C@]2(CC1)OCCc1c3cc(Br)ccc3[nH]c12</smiles>

Compound 4c

${ }^{1} \mathrm{H}$ NMR (500 MHz, DMSO)

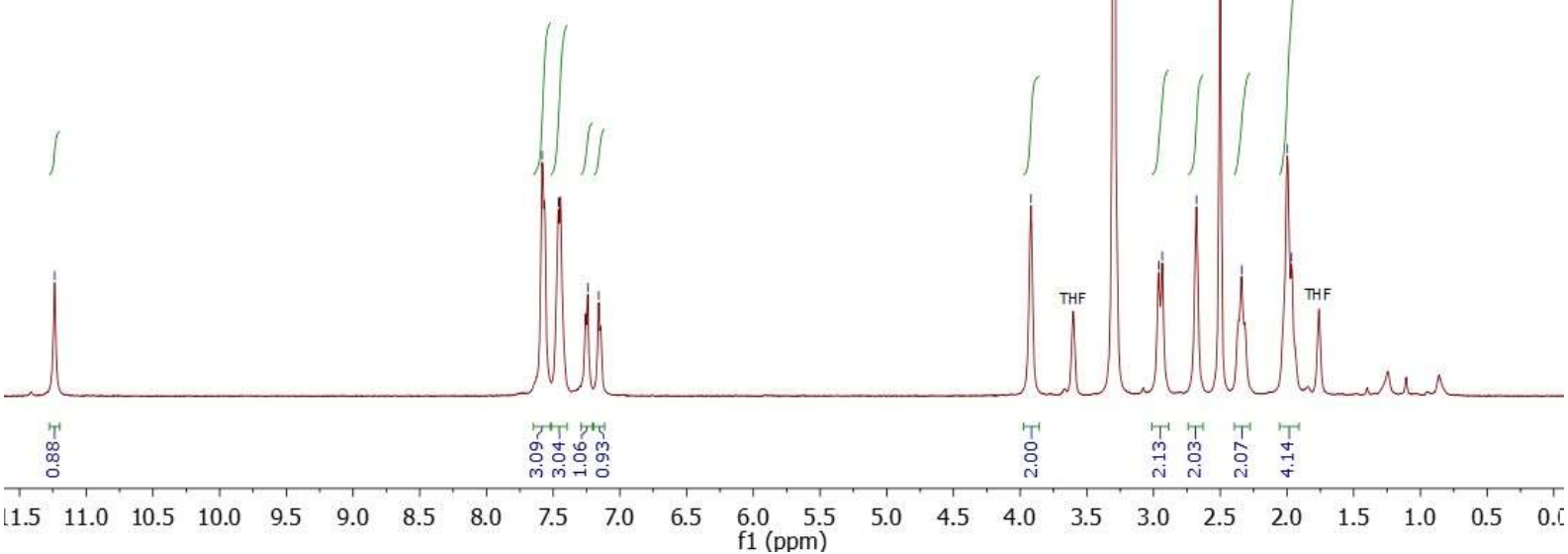

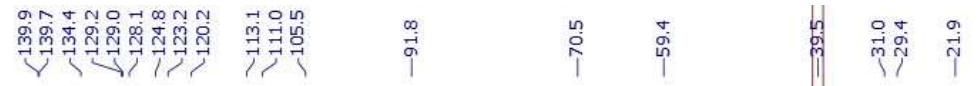

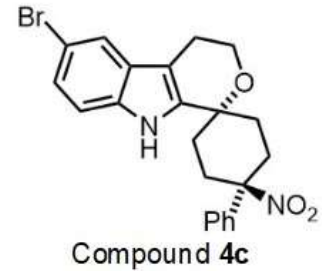

${ }^{13} \mathrm{C}\left\{{ }^{1} \mathrm{H}\right\}$ NMR (125 MHz, DMSO)

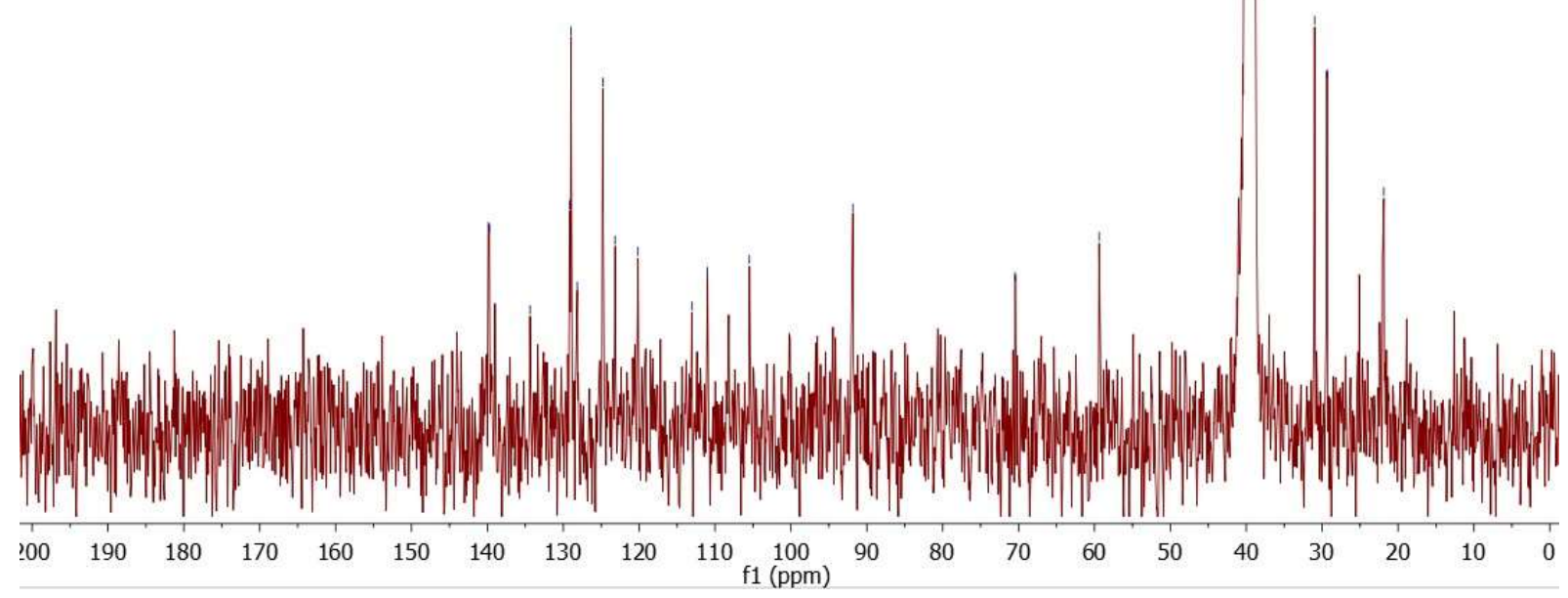




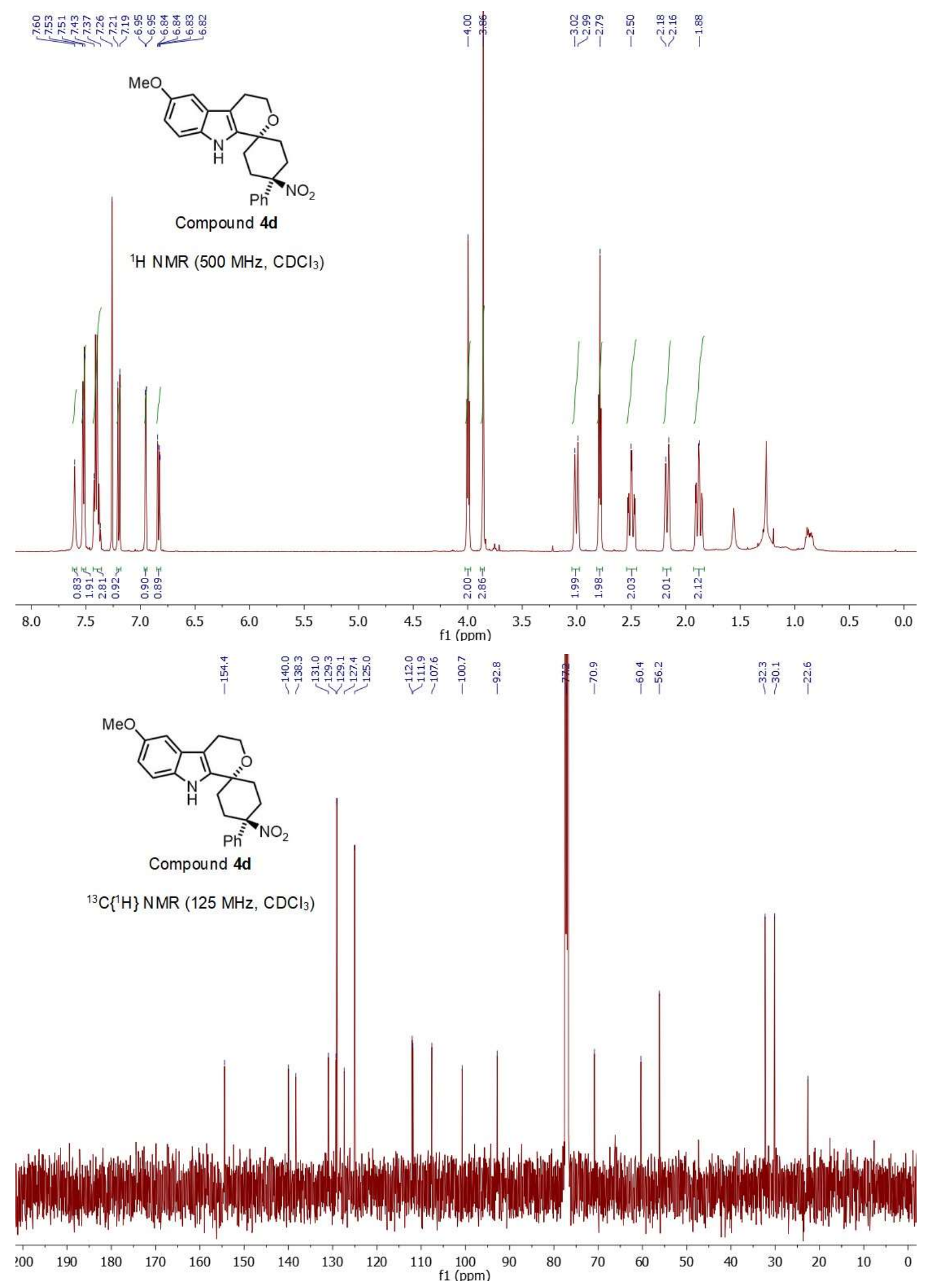




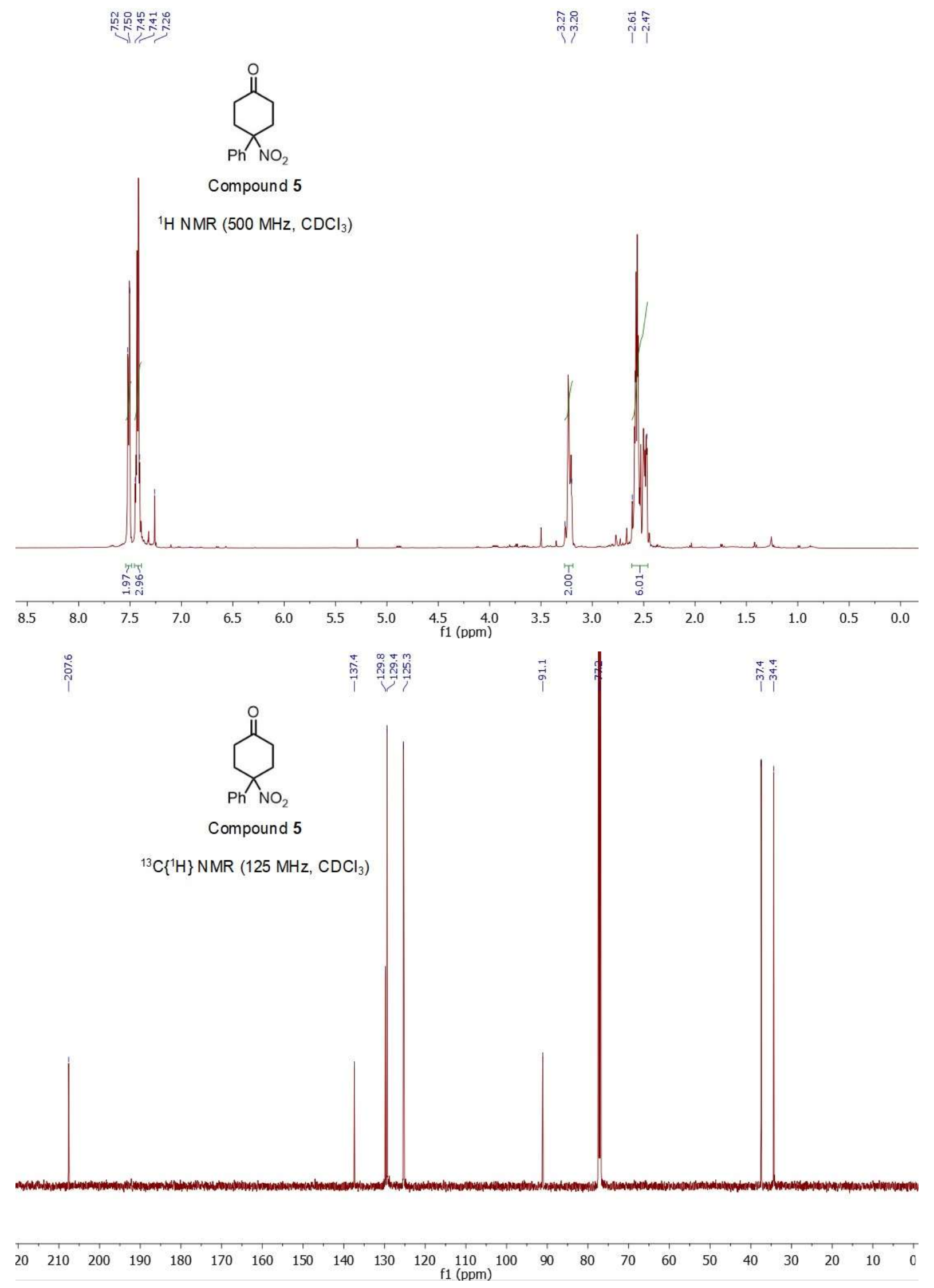




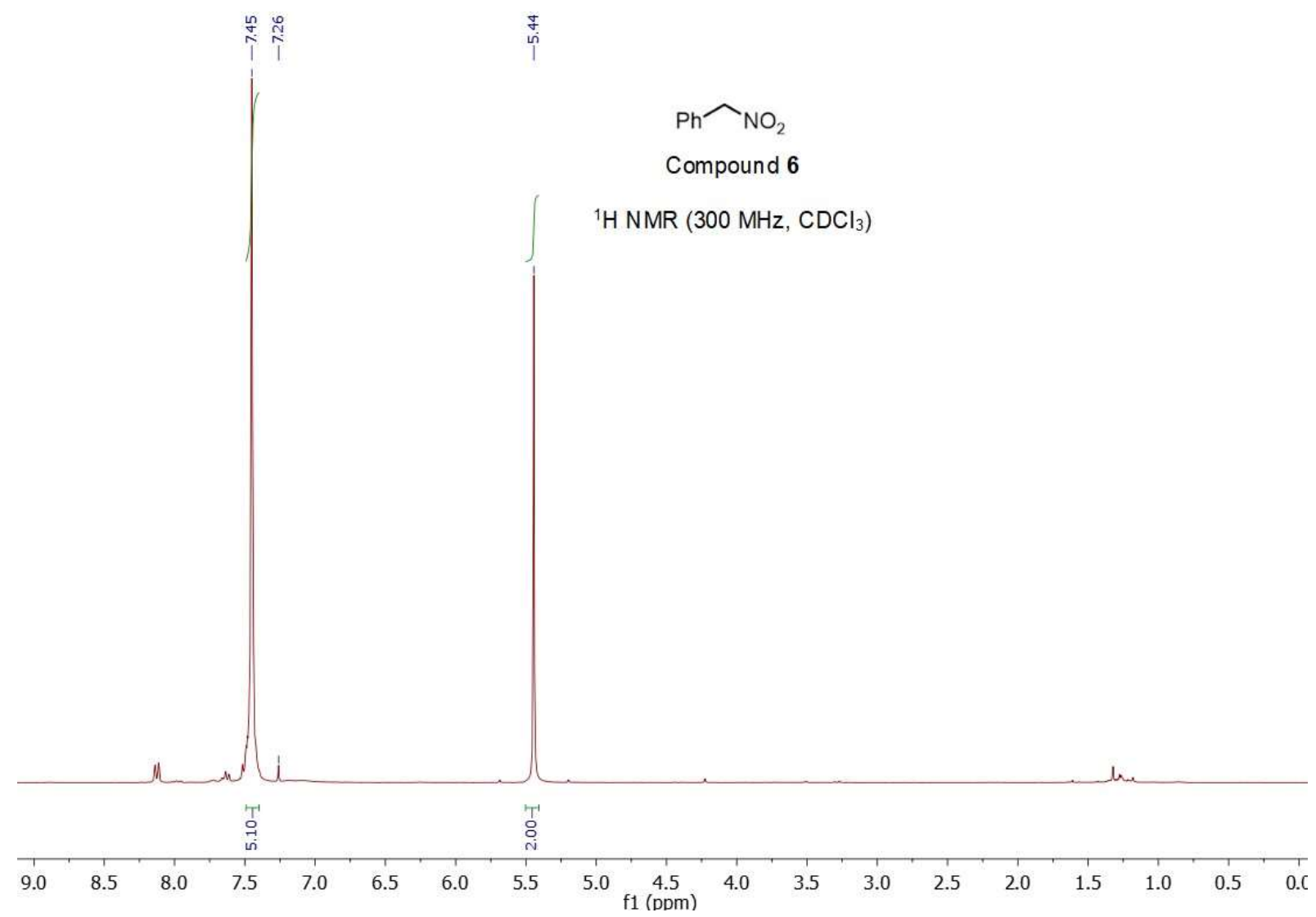




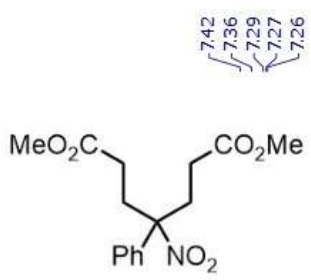

Compound 8

${ }^{1} \mathrm{H} \mathrm{NMR}\left(500 \mathrm{MHz}, \mathrm{CDCl}_{3}\right)$
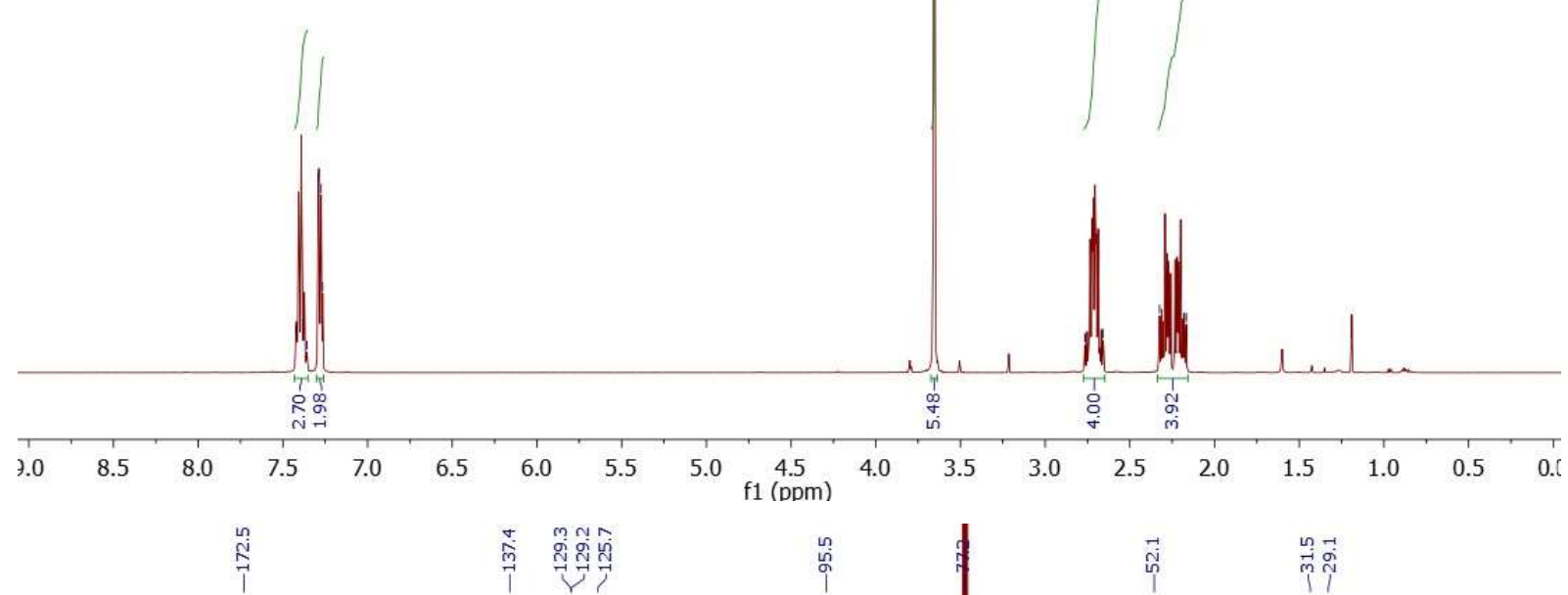

ஸึ<smiles>CC(=O)CCC(CCC(C)=O)(c1ccccc1)[N+](=O)[O-]</smiles>

Compound 8

${ }^{13} \mathrm{C}\left\{{ }^{1} \mathrm{H}\right\}$ NMR $\left(125 \mathrm{MHz}, \mathrm{CDCl}_{3}\right)$

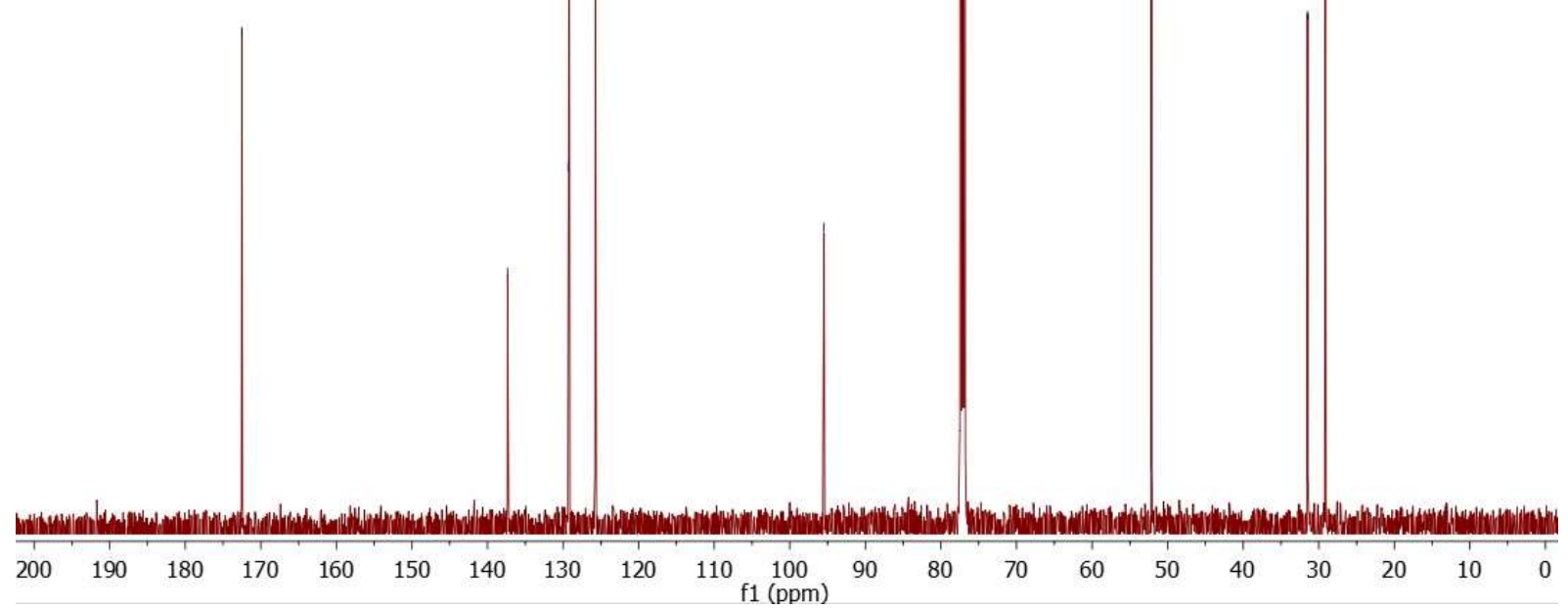


$\underset{7}{7}$

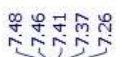

잉ำ<smiles>CC(=O)C1CC(c2ccccc2)([N+](=O)[O-])CCC1=O</smiles>

Compound 9

${ }^{1} \mathrm{H}$ NMR $\left(500 \mathrm{MHz}, \mathrm{CDCl}_{3}\right)$

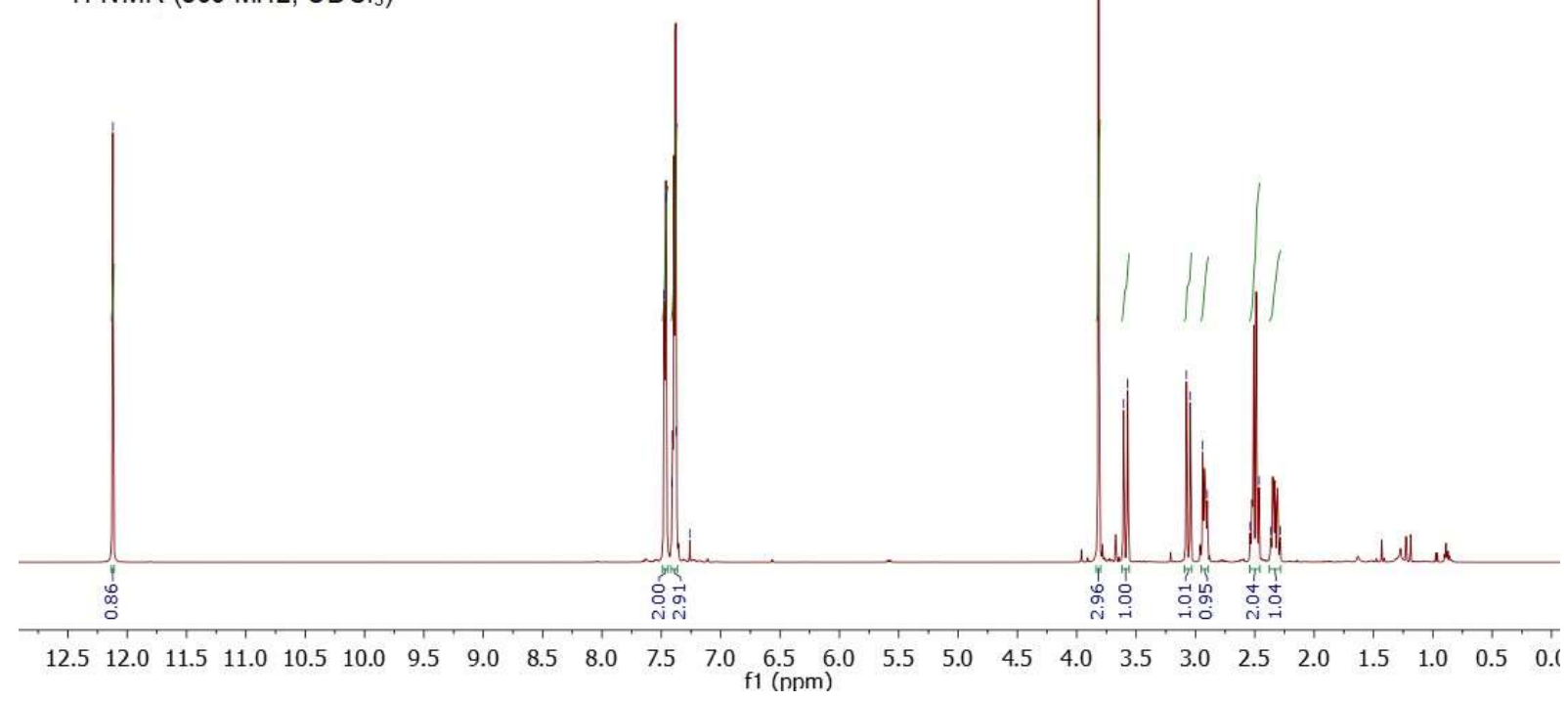

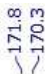

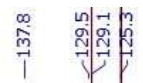

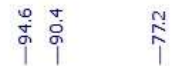

के लें<smiles>CC(=O)C1CC(c2ccccc2)([N+](=O)[O-])CCC1=O</smiles>

Compound 9

${ }^{13} \mathrm{C}\left\{{ }^{1} \mathrm{H}\right\}$ NMR $\left(125 \mathrm{MHz}, \mathrm{CDCl}_{3}\right)$

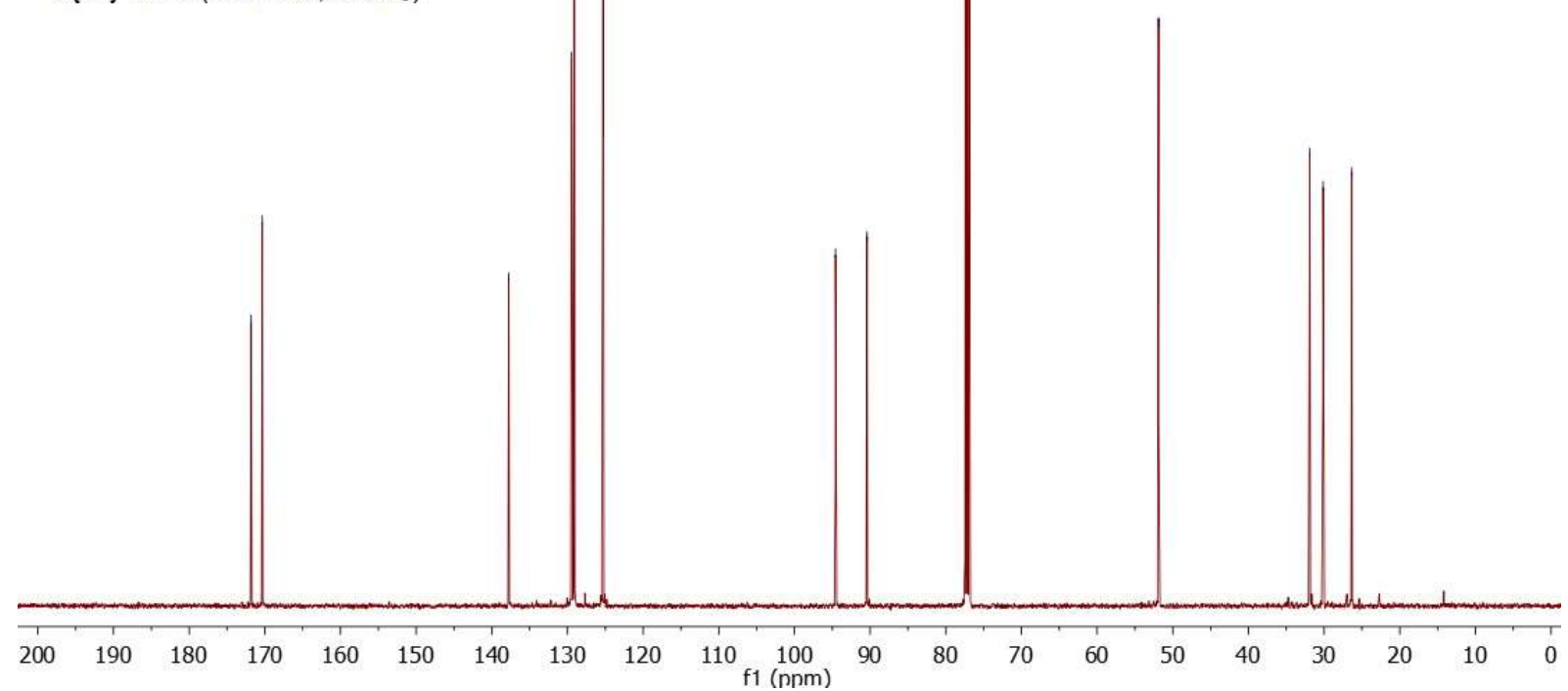




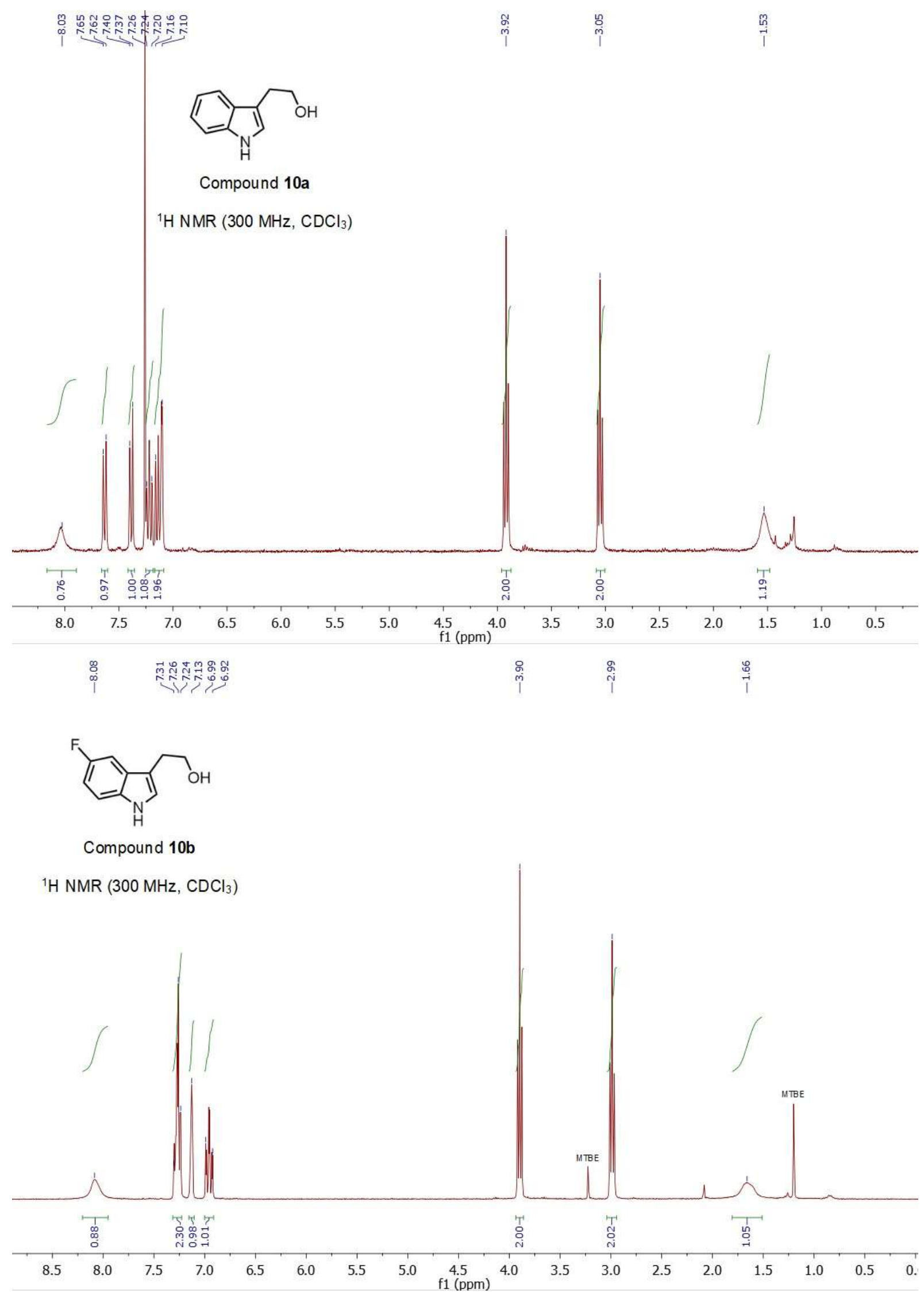


<smiles>OCCc1c[nH]c2ccc(Br)cc12</smiles>

\section{Compound 10c}

${ }^{1} \mathrm{H} \mathrm{NMR}\left(300 \mathrm{MHz}, \mathrm{CDCl}_{3}\right)$

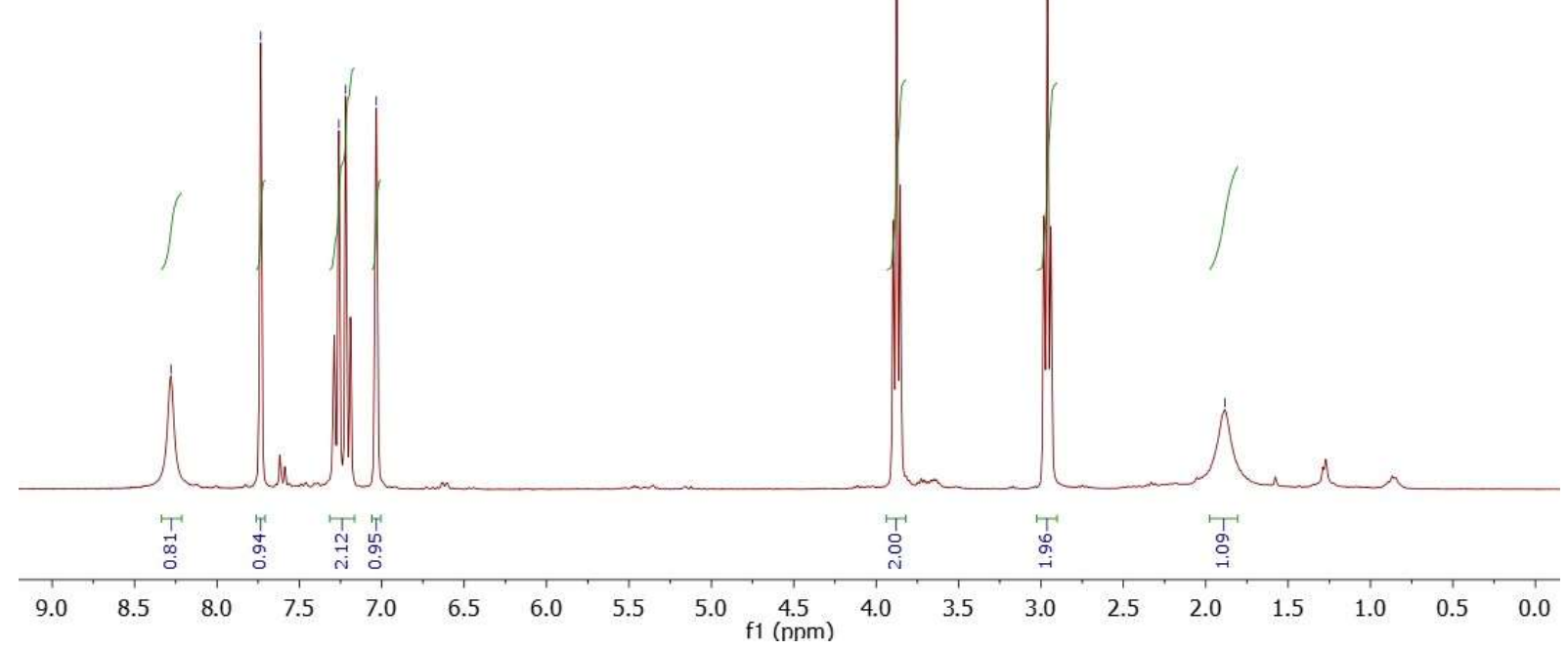
1<smiles>COc1ccc2[nH]cc(CCO)c2c1</smiles>

Compound 10d

${ }^{1} \mathrm{H} \mathrm{NMR}\left(300 \mathrm{MHz}, \mathrm{CDCl}_{3}\right)$

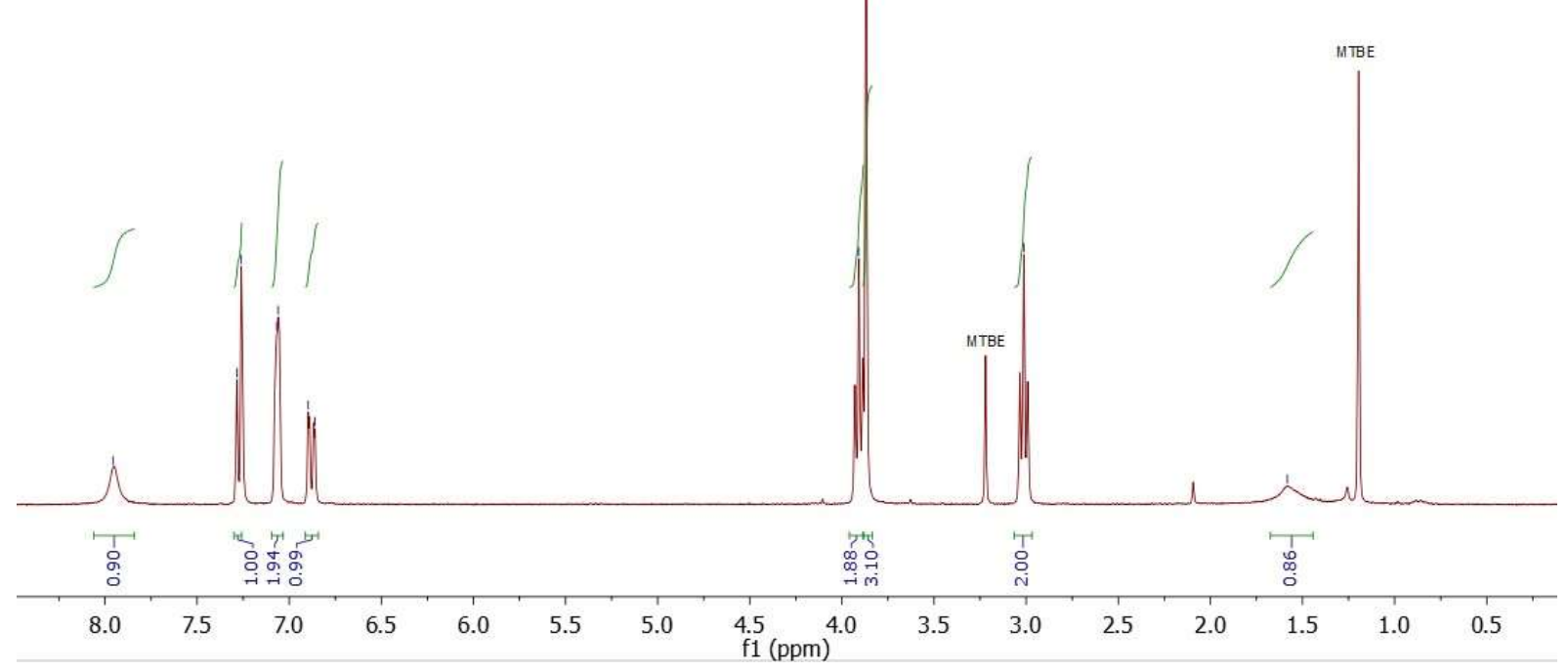




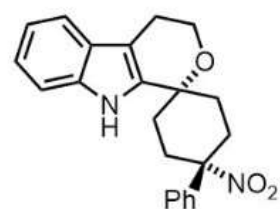

Compound 11a

${ }^{1} \mathrm{H} \mathrm{NMR}\left(500 \mathrm{MHz}, \mathrm{CDCl}_{3}\right)$

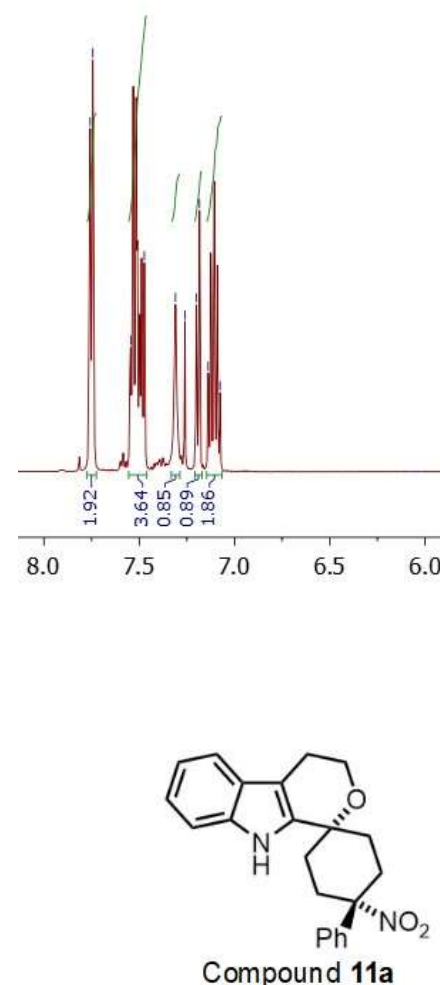

${ }^{13} \mathrm{C}\left\{{ }^{1} \mathrm{H}\right\}$ NMR $\left(125 \mathrm{MHz}, \mathrm{CDCl}_{3}\right)$

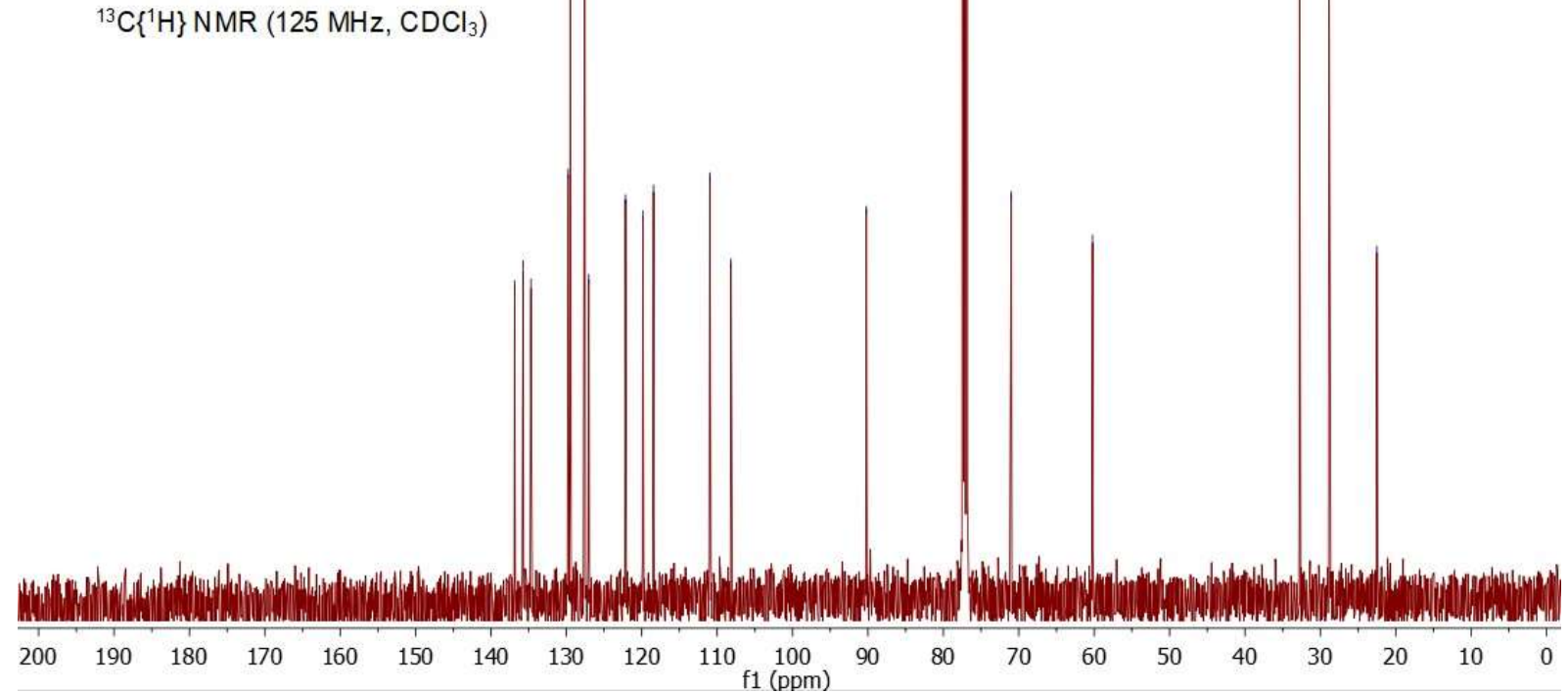




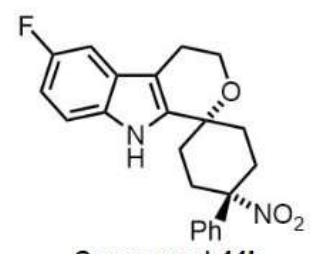

Compound 11b

${ }^{1} \mathrm{H}$ NMR $(500 \mathrm{MHz}, \mathrm{DMSO})$

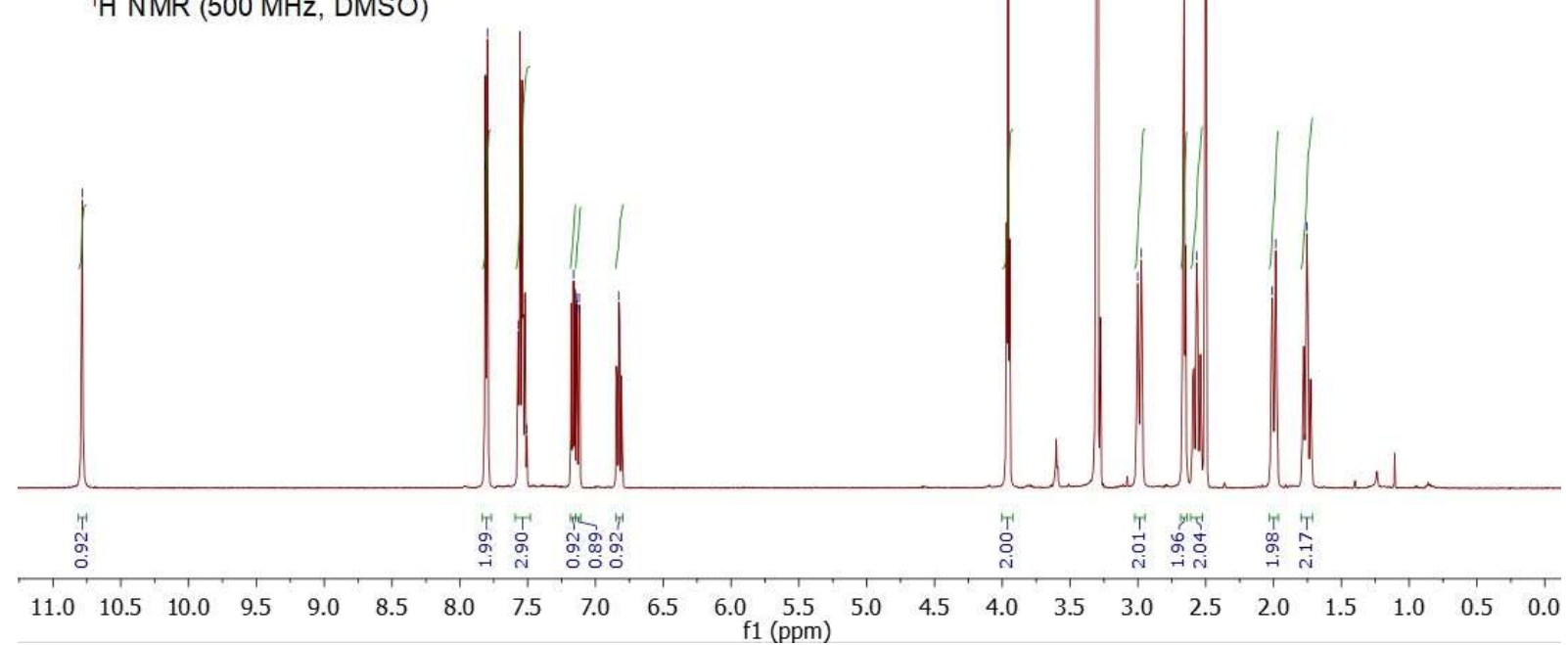

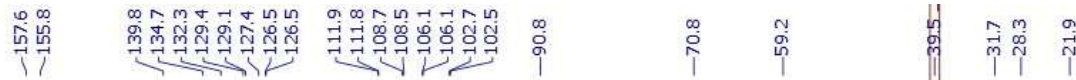

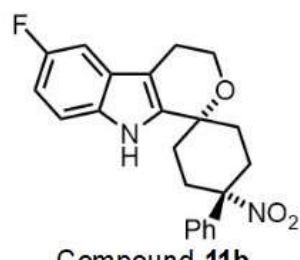

${ }^{13} \mathrm{C}\left\{{ }^{1} \mathrm{H}\right\}$ NMR (125 MHz, DMSO)

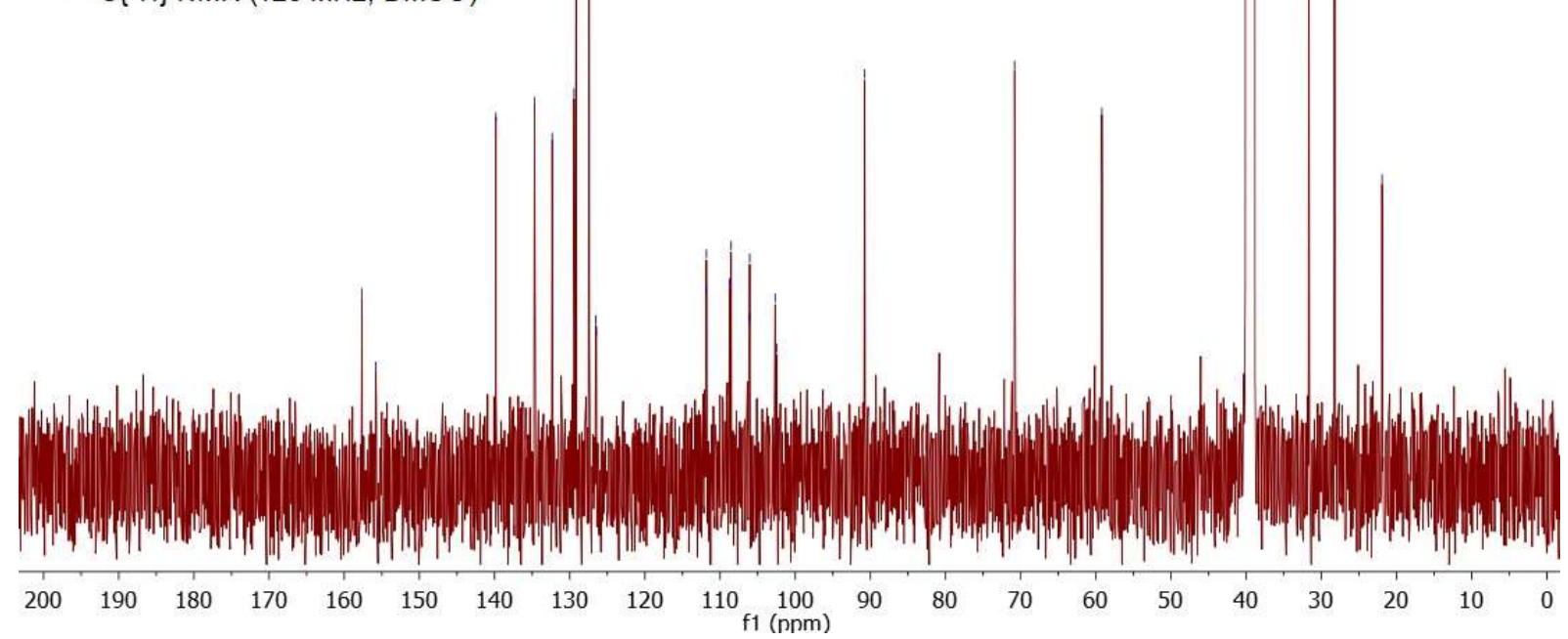




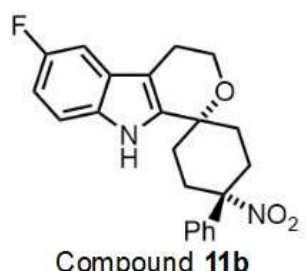

${ }^{19} \mathrm{~F}\left\{{ }^{1} \mathrm{H}\right\}$ NMR (470 MHz, DMSO)

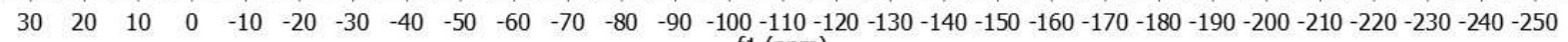
f1 (ppm) 


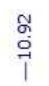<smiles></smiles>

Compound 11c

${ }^{1} \mathrm{H}$ NMR $(500 \mathrm{MHz}, \mathrm{DMSO})$

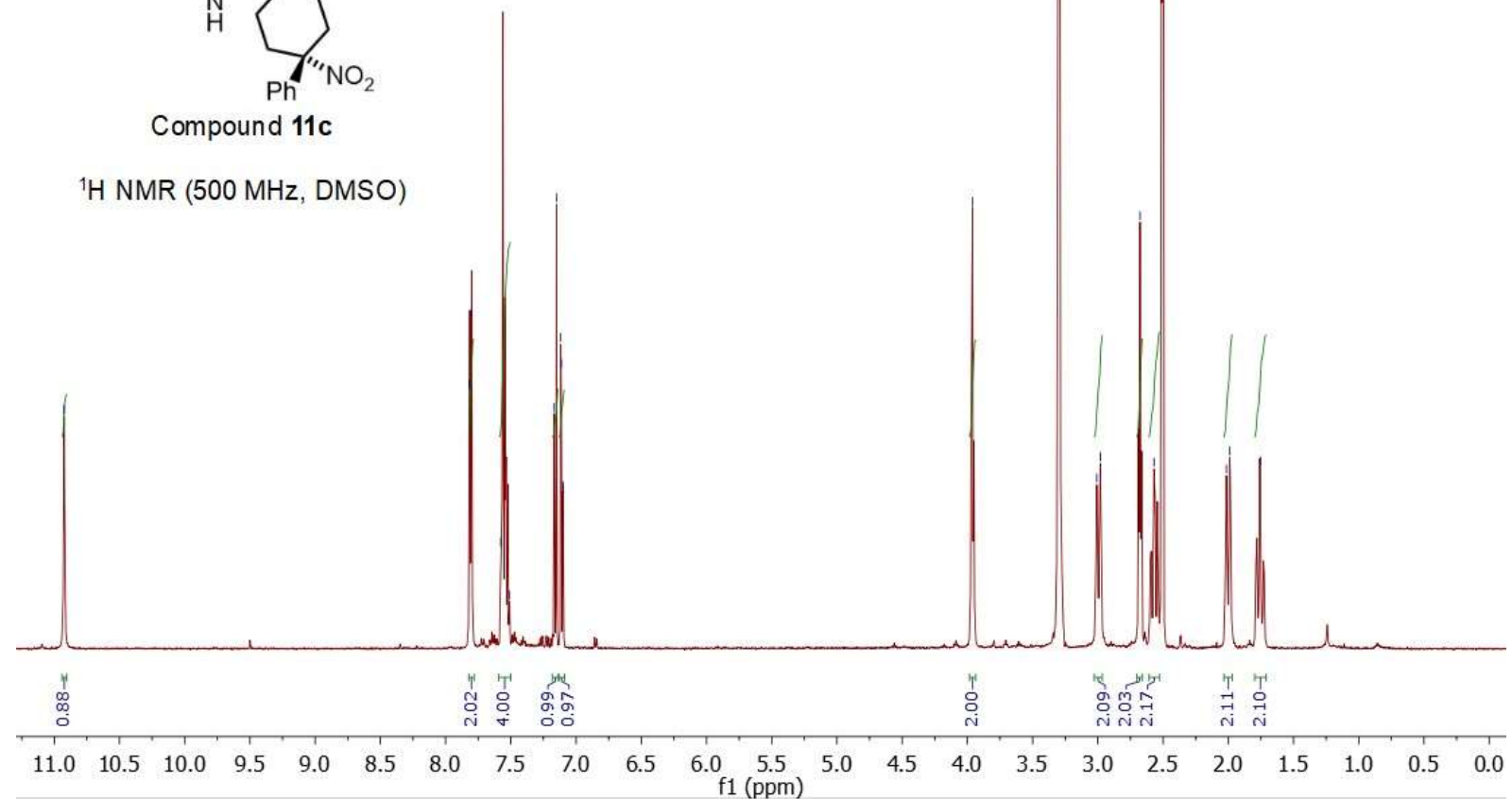

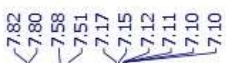

Compound 11c

${ }^{13} \mathrm{C}\left\{{ }^{1} \mathrm{H}\right\}$ NMR $(125 \mathrm{MHz}, \mathrm{DMSO})$

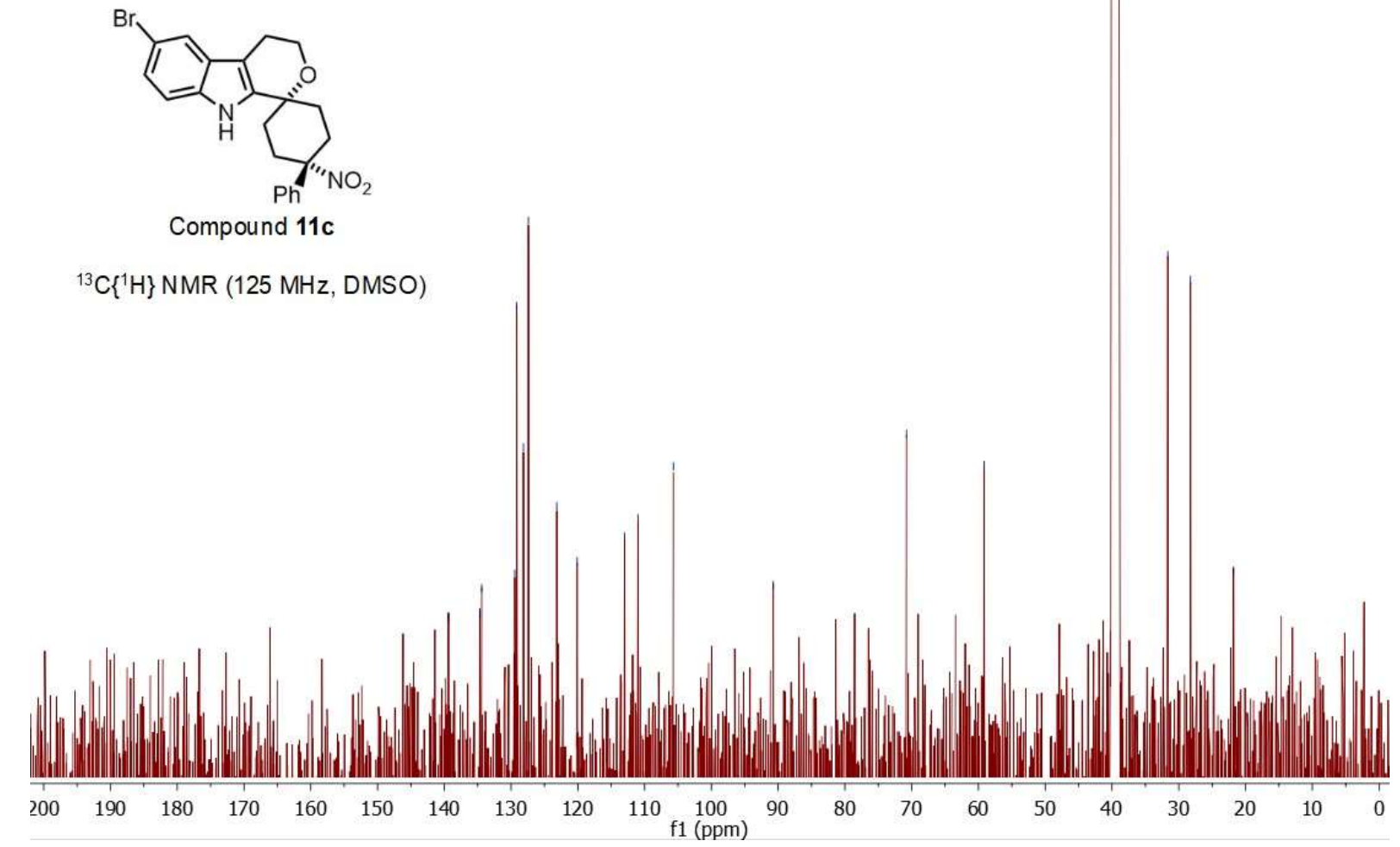


<smiles>N[C@]1(c2ccccc2)CC[C@]2(CC1)OCCc1c3ccccc3[nH]c12</smiles>

Compound 12

${ }^{1} \mathrm{H}$ NMR $\left(500 \mathrm{MHz}, \mathrm{CDCl}_{3}\right)$
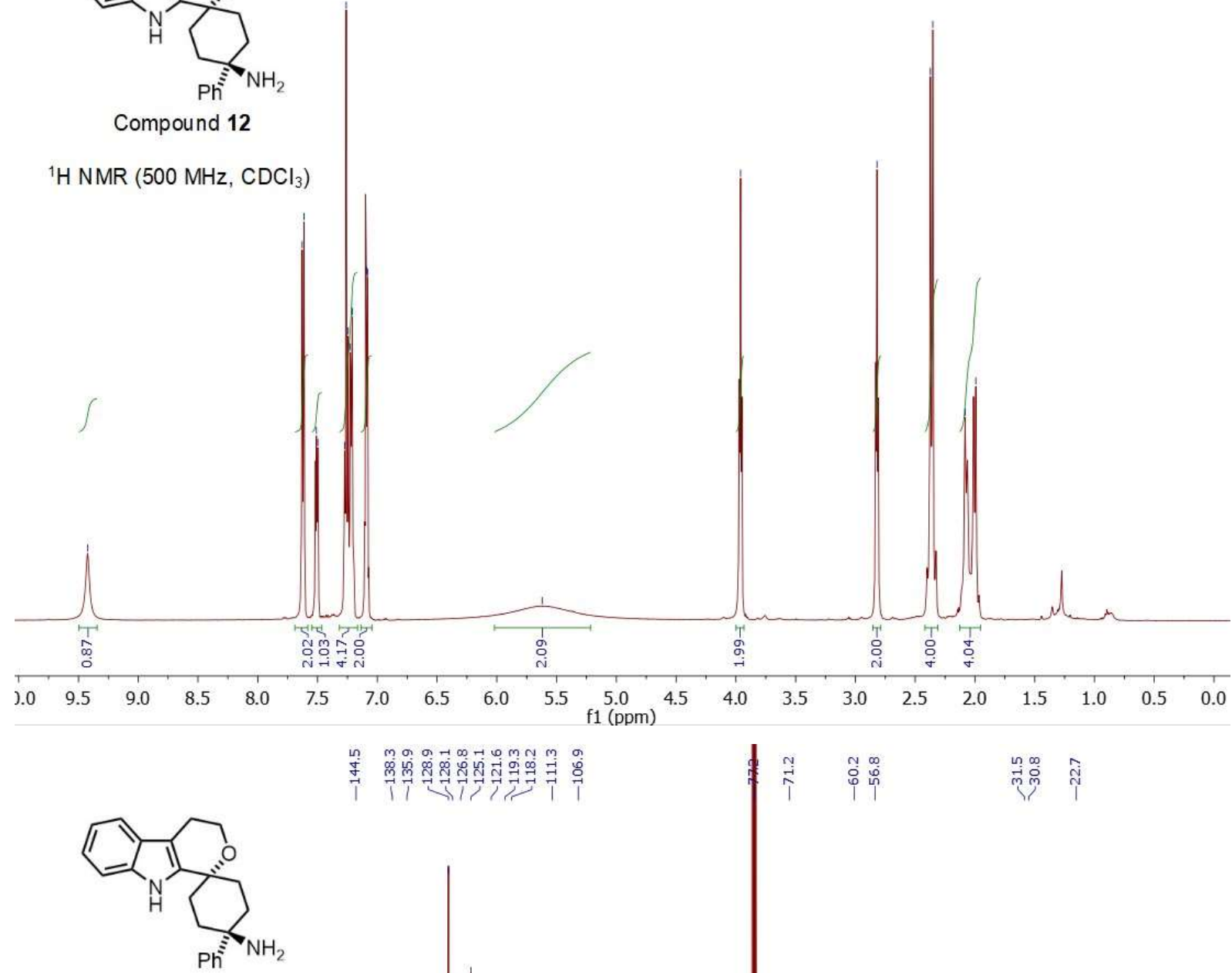

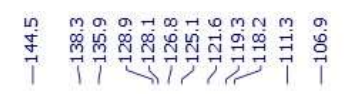

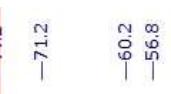

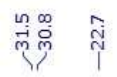

Compound 12

${ }^{13} \mathrm{C}\left\{{ }^{1} \mathrm{H}\right\}$ NMR $\left(125 \mathrm{MHz}, \mathrm{CDCl}_{3}\right)$

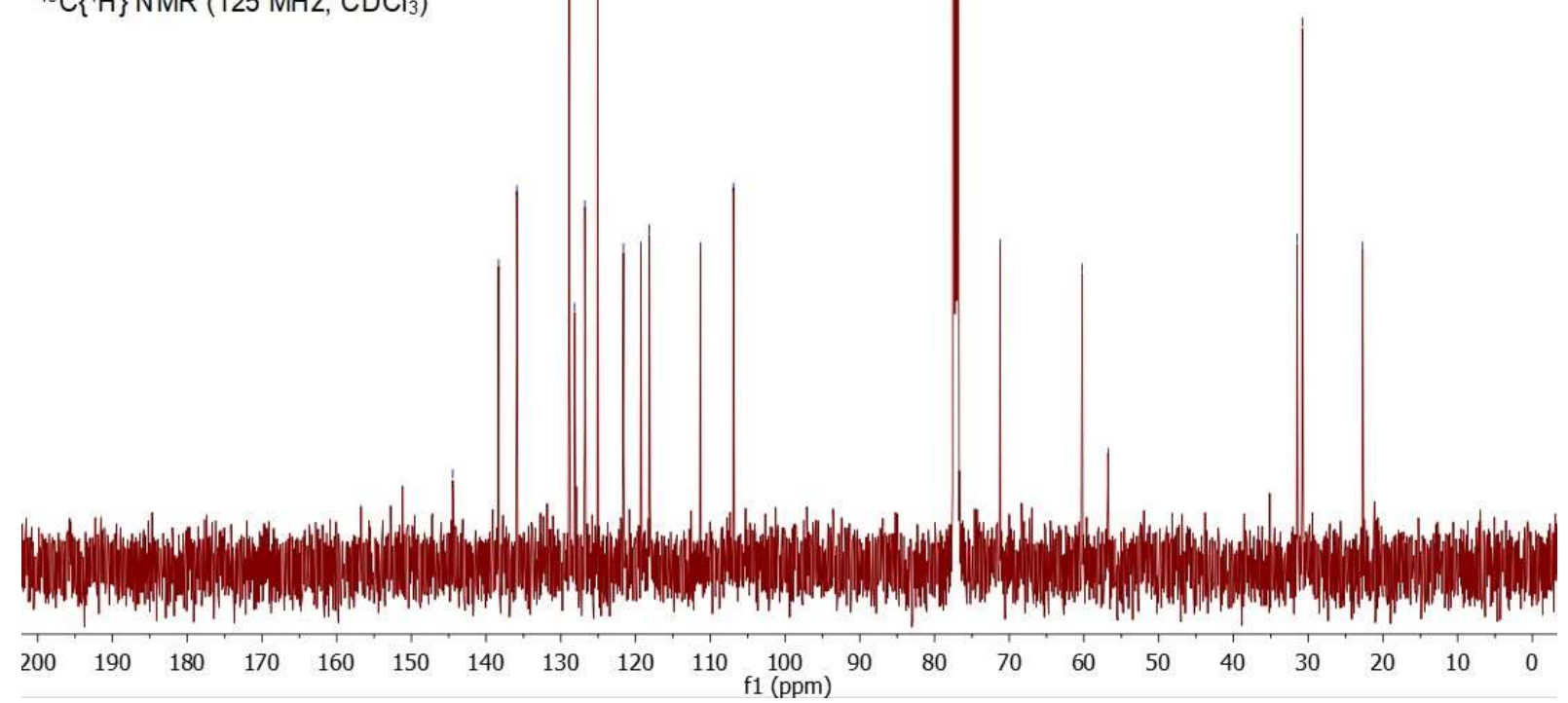


$\stackrel{\infty}{\infty}$<smiles>CNC[C@]1(c2ccccc2)CC[C@]2(CC1)OCCc1c3ccccc3[nH]c12</smiles>

Compound 13

${ }^{1} \mathrm{H}$ NMR (500 MHz, DMSO)
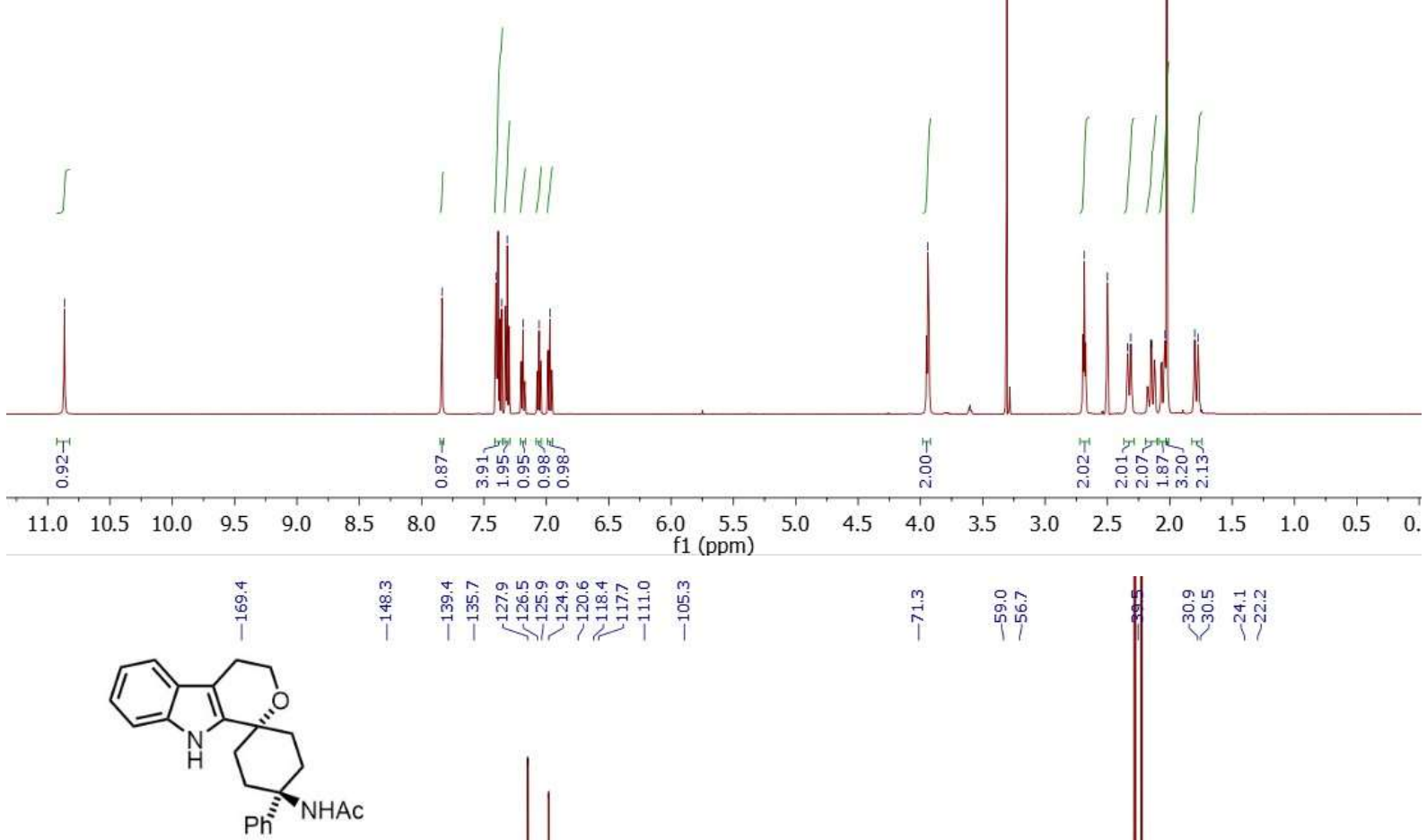

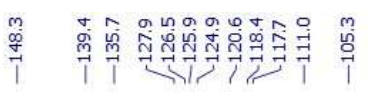

$\stackrel{m}{\rightarrow}$ फं क्षे

midn

Compound 13

${ }^{13} \mathrm{C}\left\{{ }^{1} \mathrm{H}\right\}$ NMR $(125 \mathrm{MHz}$, DMSO)

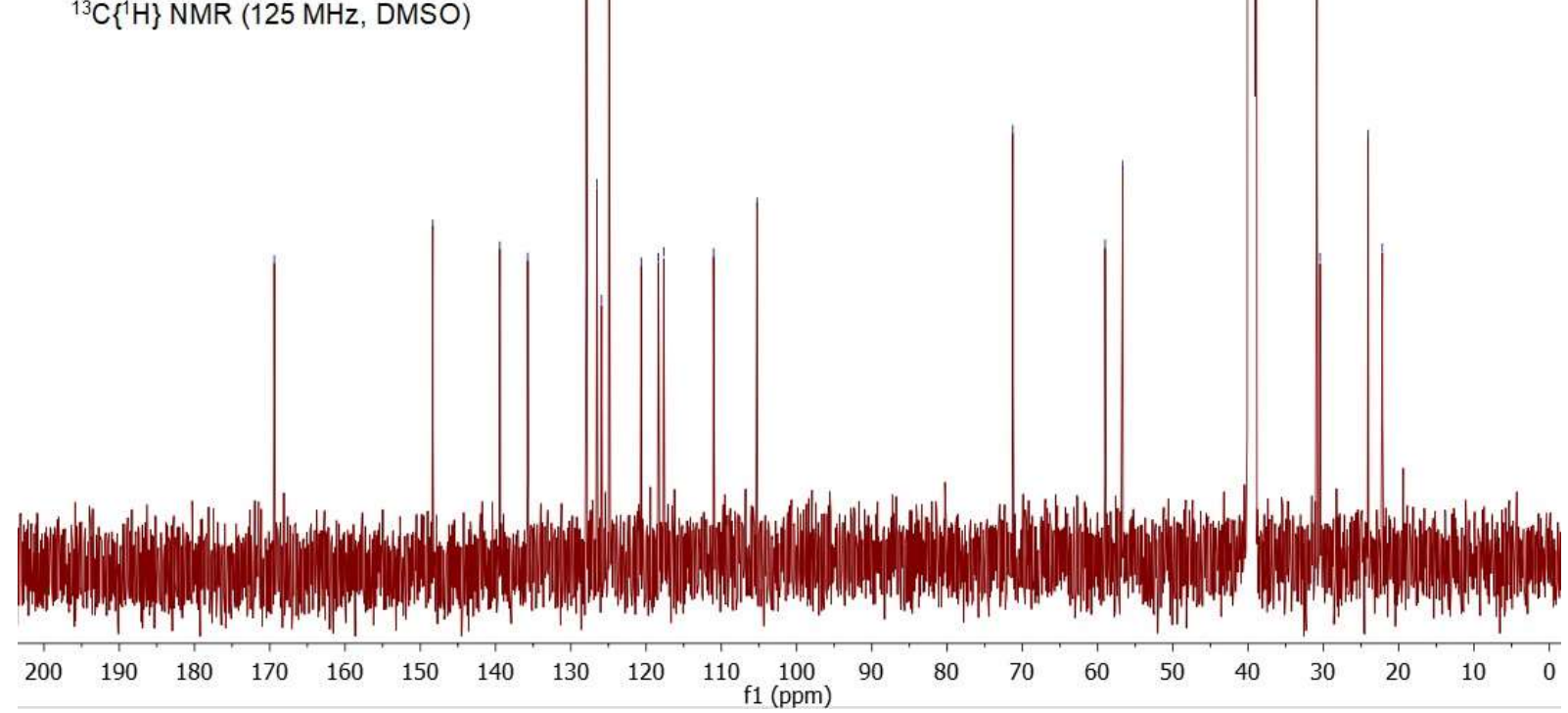




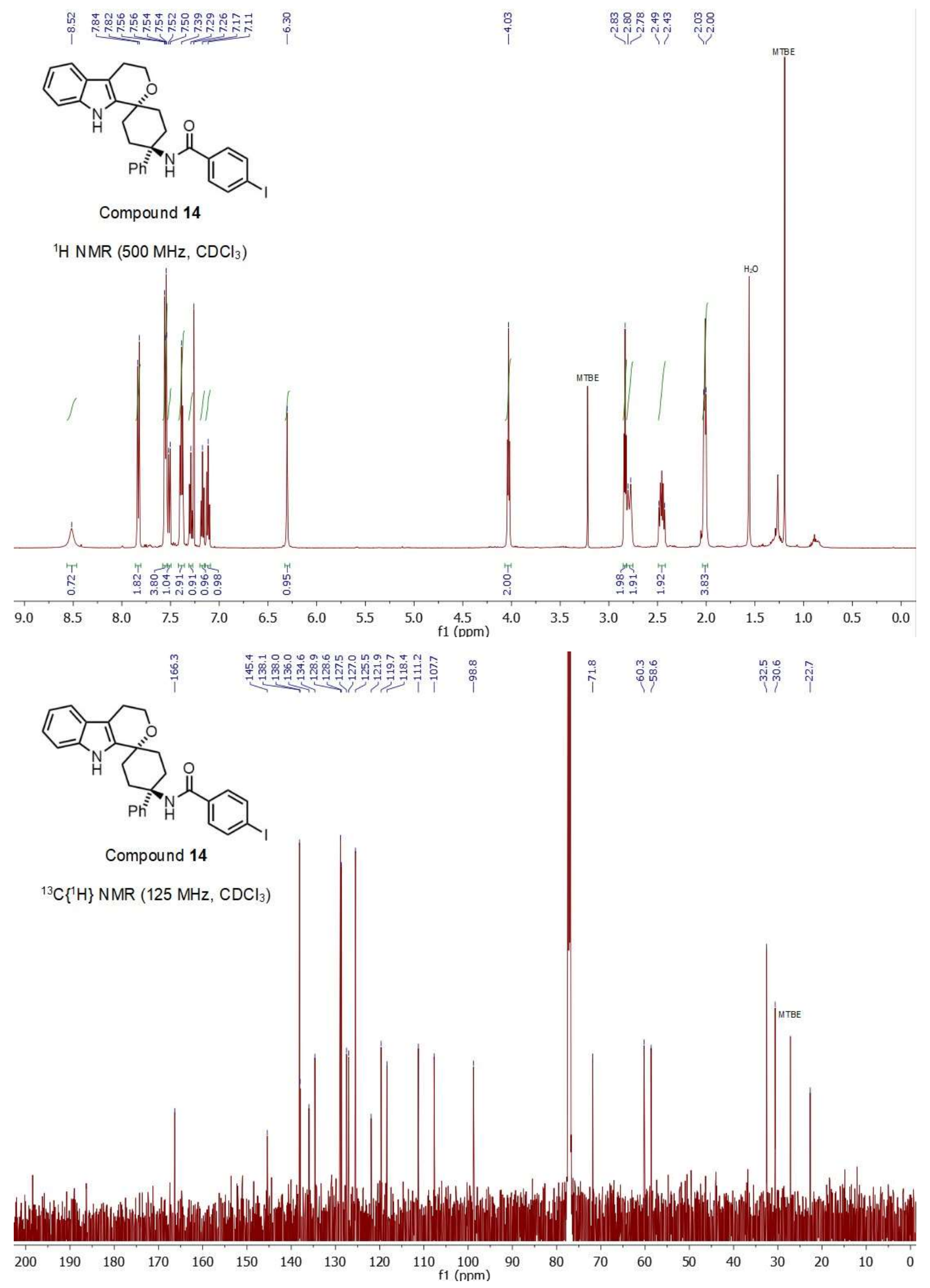




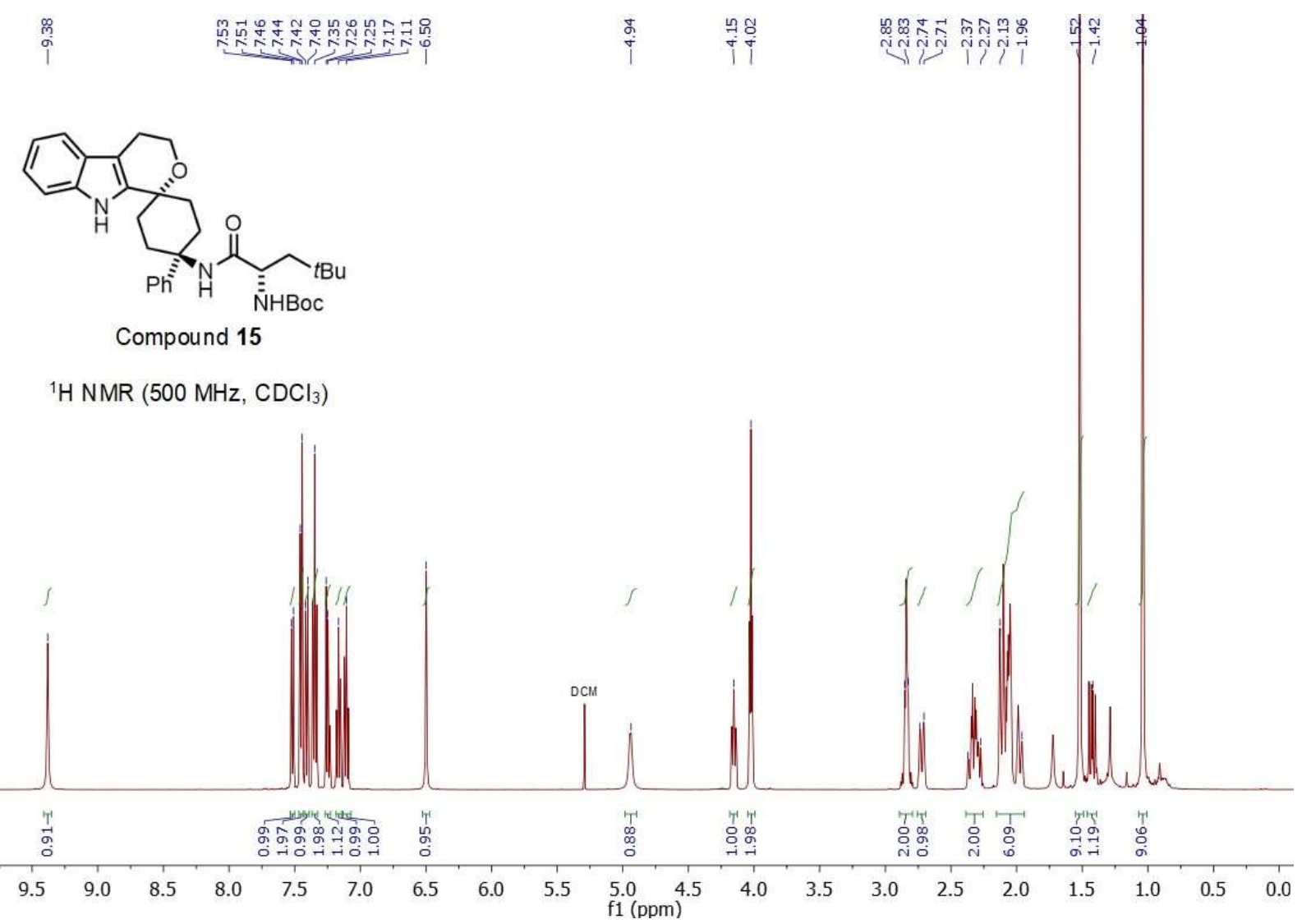

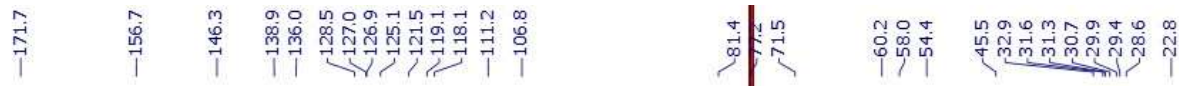

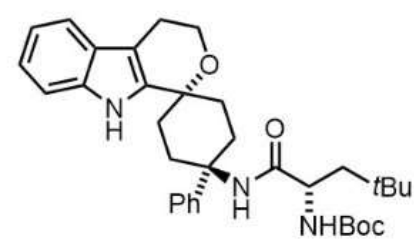

Compound 15

${ }^{13} \mathrm{C}\left\{{ }^{1} \mathrm{H}\right\} \mathrm{NMR}\left(125 \mathrm{MHz}, \mathrm{CDCl}_{3}\right)$

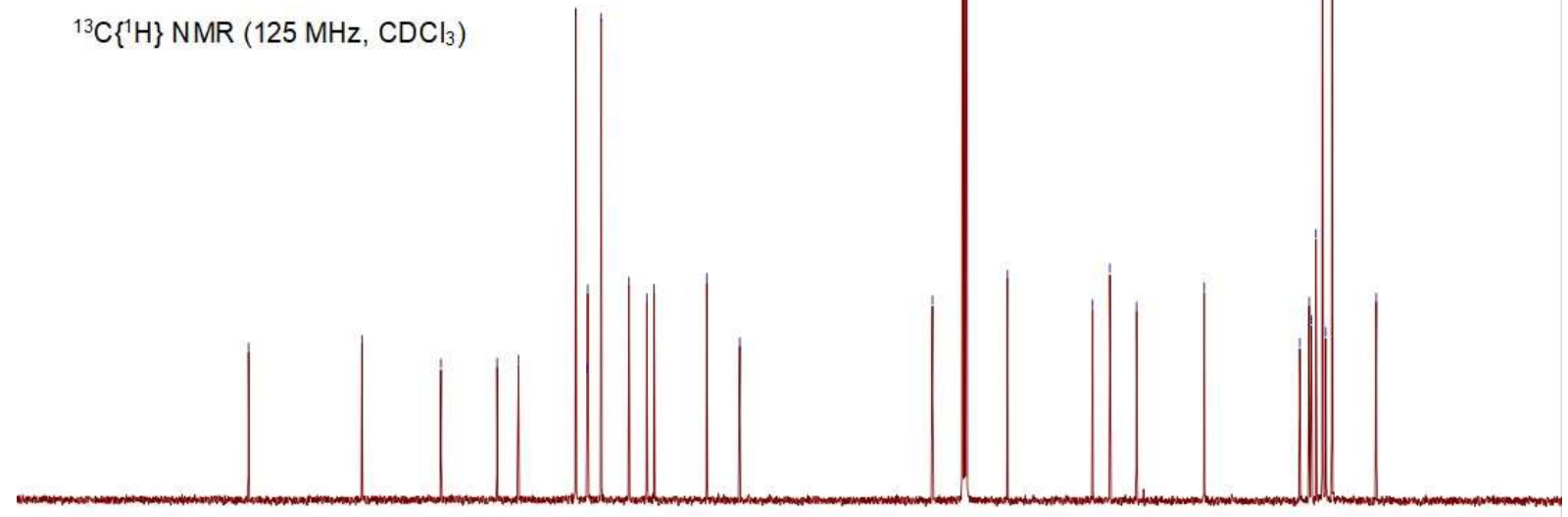

$\begin{array}{lllllllllllllllllllll}200 & 190 & 180 & 170 & 160 & 150 & 140 & 130 & 120 & 110 & 100 & 90 & 80 & 70 & 60 & 50 & 40 & 30 & 20 & 10 & 0\end{array}$ 


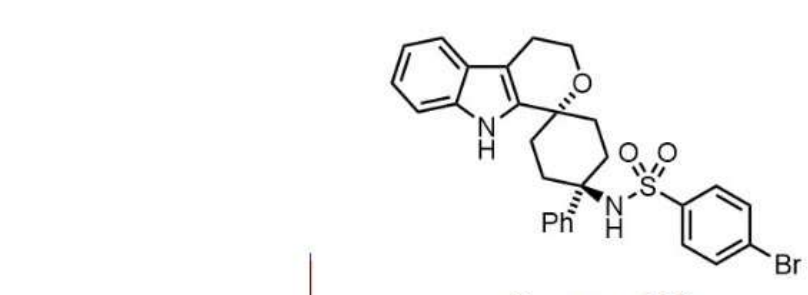

Compound 16

${ }^{1} \mathrm{H}$ NMR $\left(500 \mathrm{MHz}, \mathrm{CDCl}_{3}\right)$

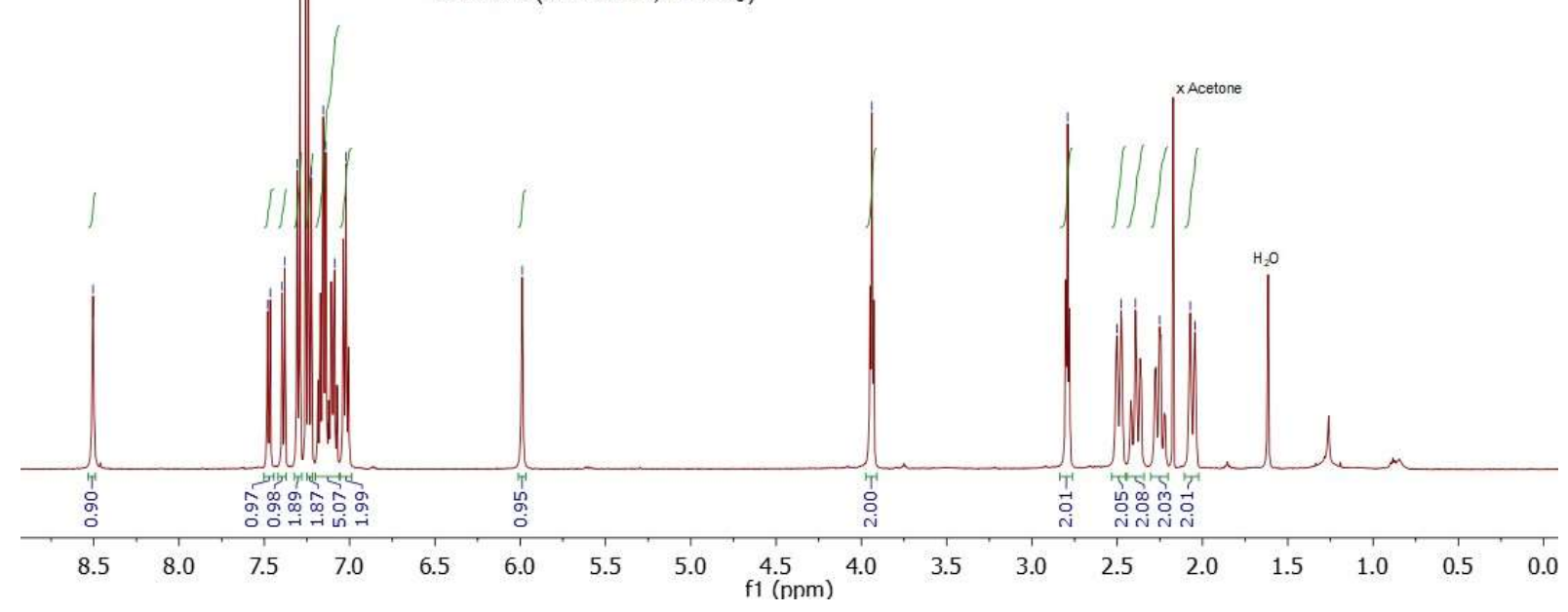

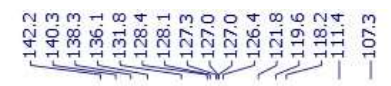

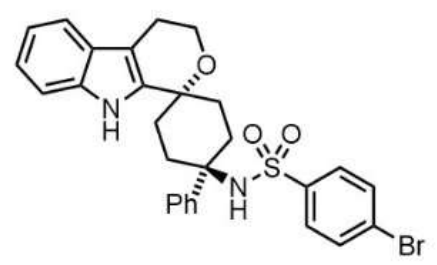

Compound 16

${ }^{13} \mathrm{C}\left\{{ }^{1} \mathrm{H}\right\}$ NMR $\left(125 \mathrm{MHz}, \mathrm{CDCl}_{3}\right)$

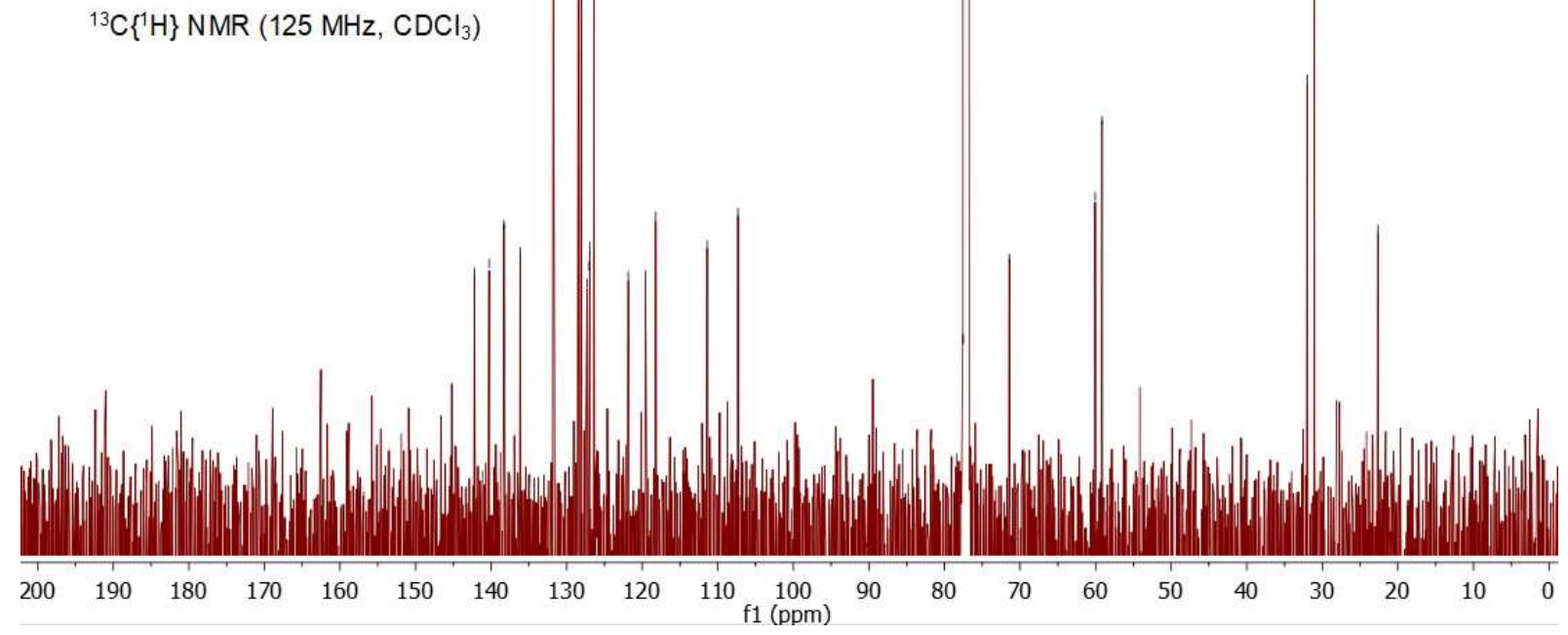




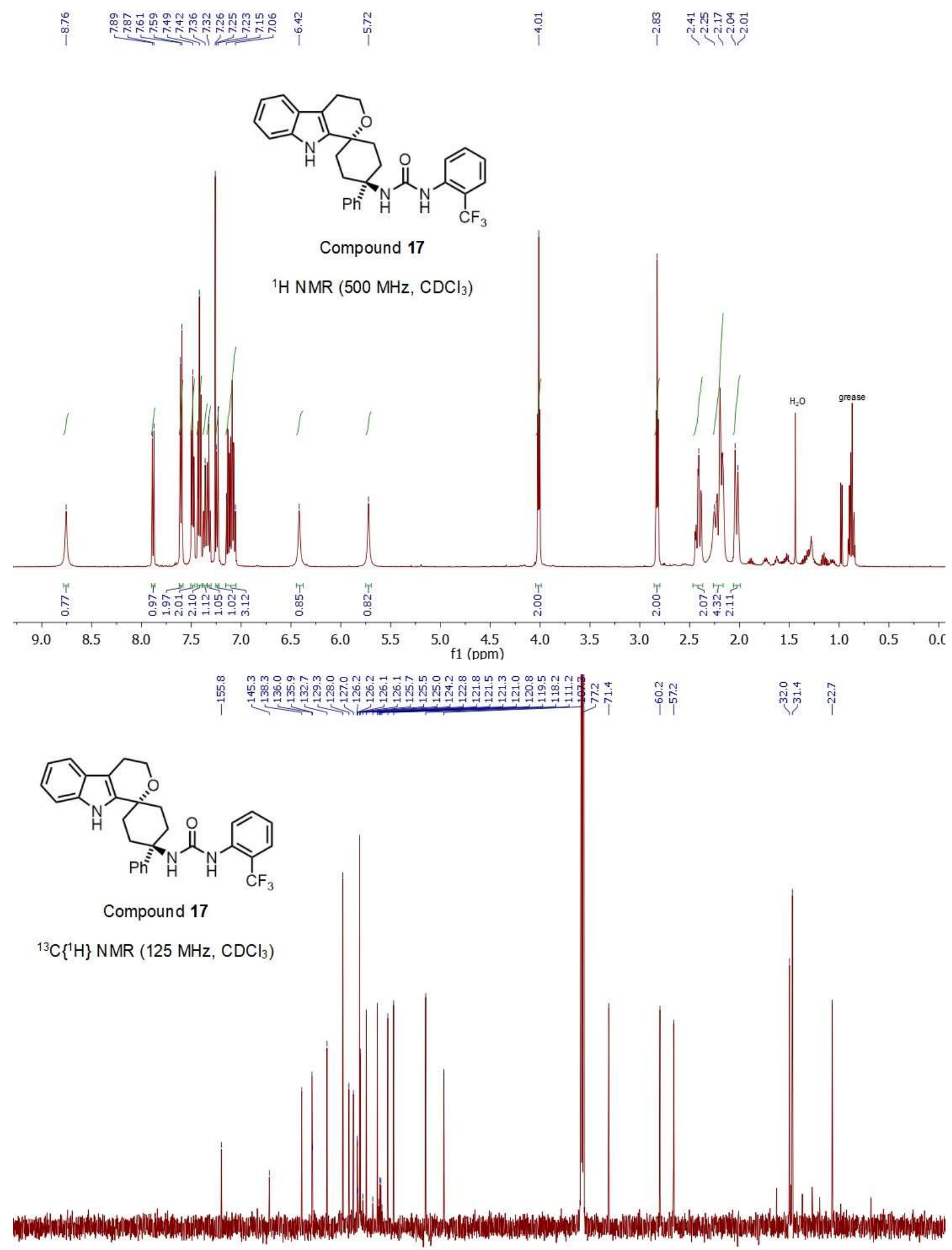

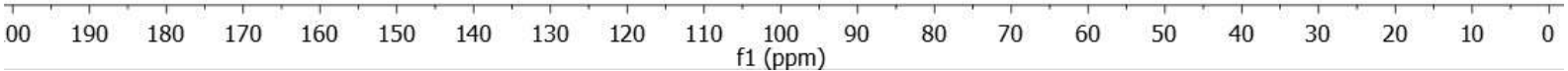




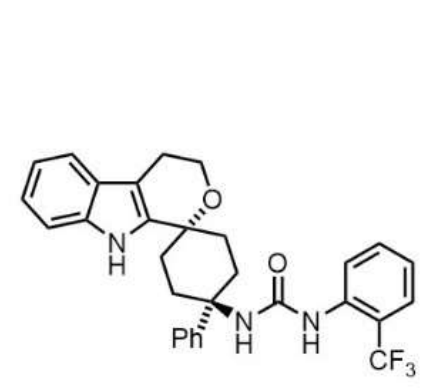

Compound 17

${ }^{19} \mathrm{~F}\left\{{ }^{1} \mathrm{H}\right\} \mathrm{NMR}\left(470 \mathrm{MHz}, \mathrm{CDCl}_{3}\right)$

$\begin{array}{llllllllllllll}30 & 20 & 10 & 0 & -10 & -20 & -30 & -40 & -50 & -60 & -70 & -80 & -90 & -100\end{array}-110-120-130-140-150-160-170-180-190-200-210-220-230-240-250$ 


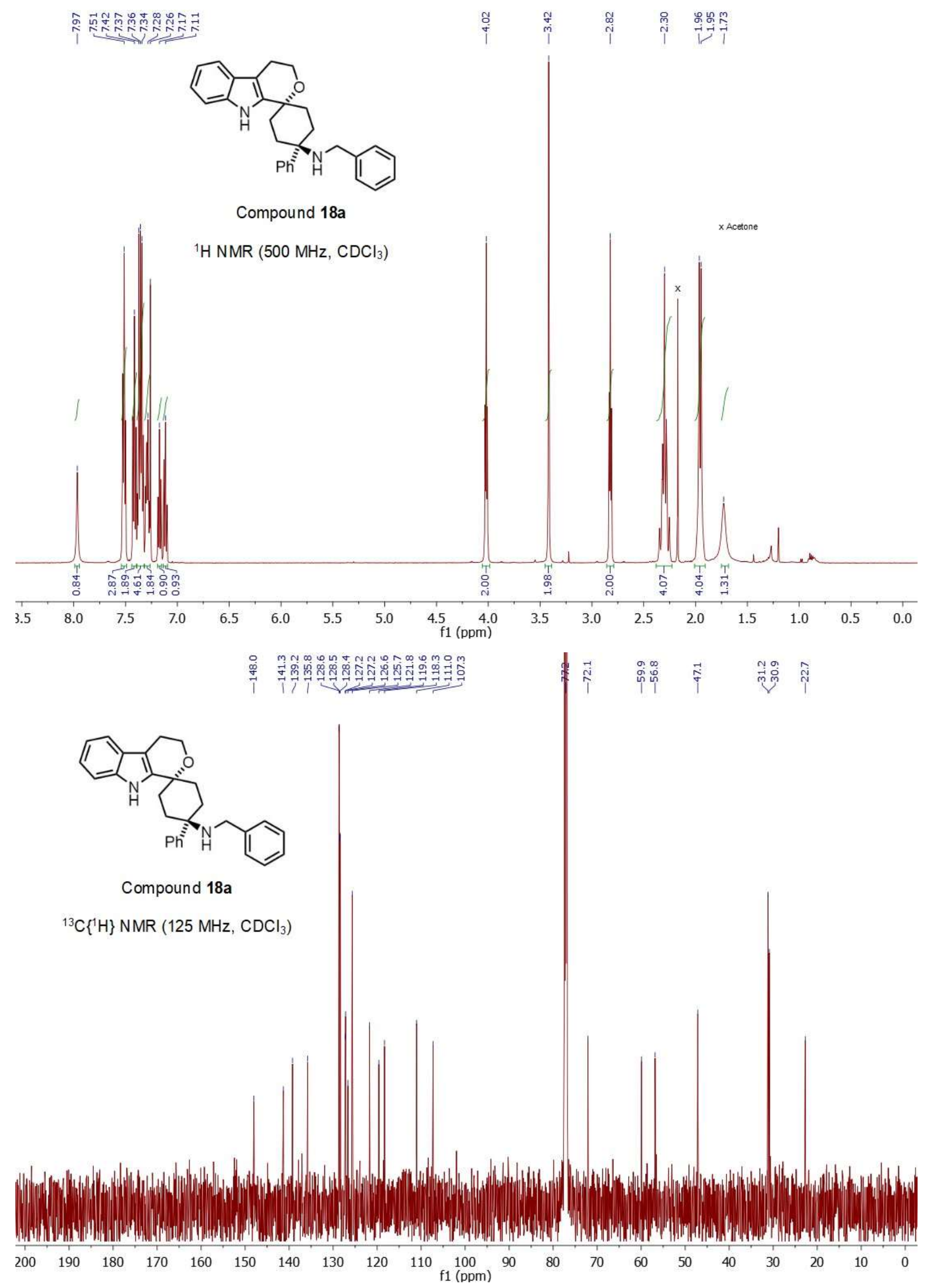




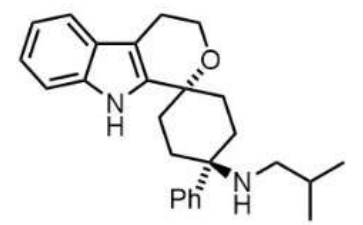

Compound $\mathbf{1 8 b}$

${ }^{1} \mathrm{H}$ NMR $\left(500 \mathrm{MHz}, \mathrm{CDCl}_{3}\right)$

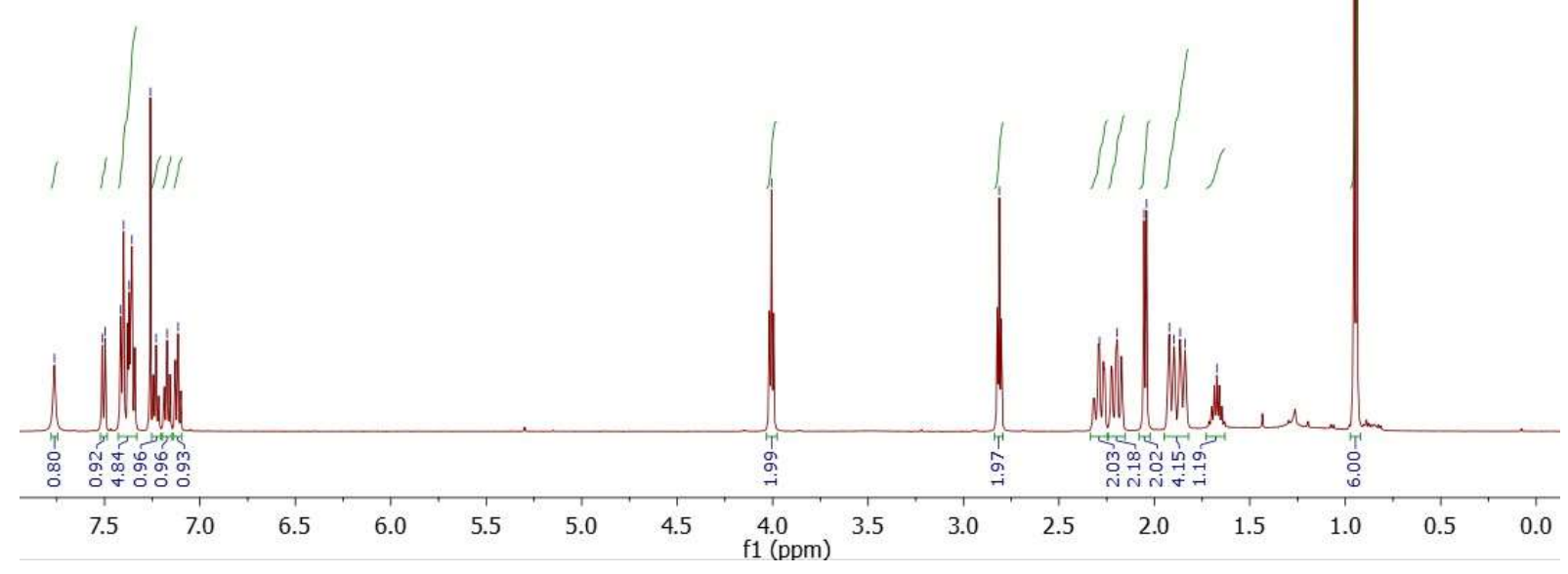

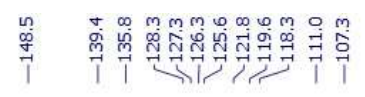

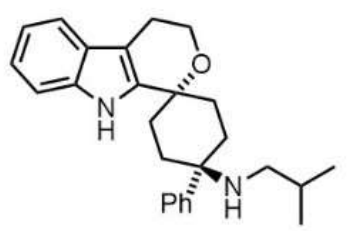

Compound $18 \mathrm{~b}$

${ }^{13} \mathrm{C}\left\{{ }^{1} \mathrm{H}\right\}$ NMR $\left(125 \mathrm{MHz}, \mathrm{CDCl}_{3}\right)$

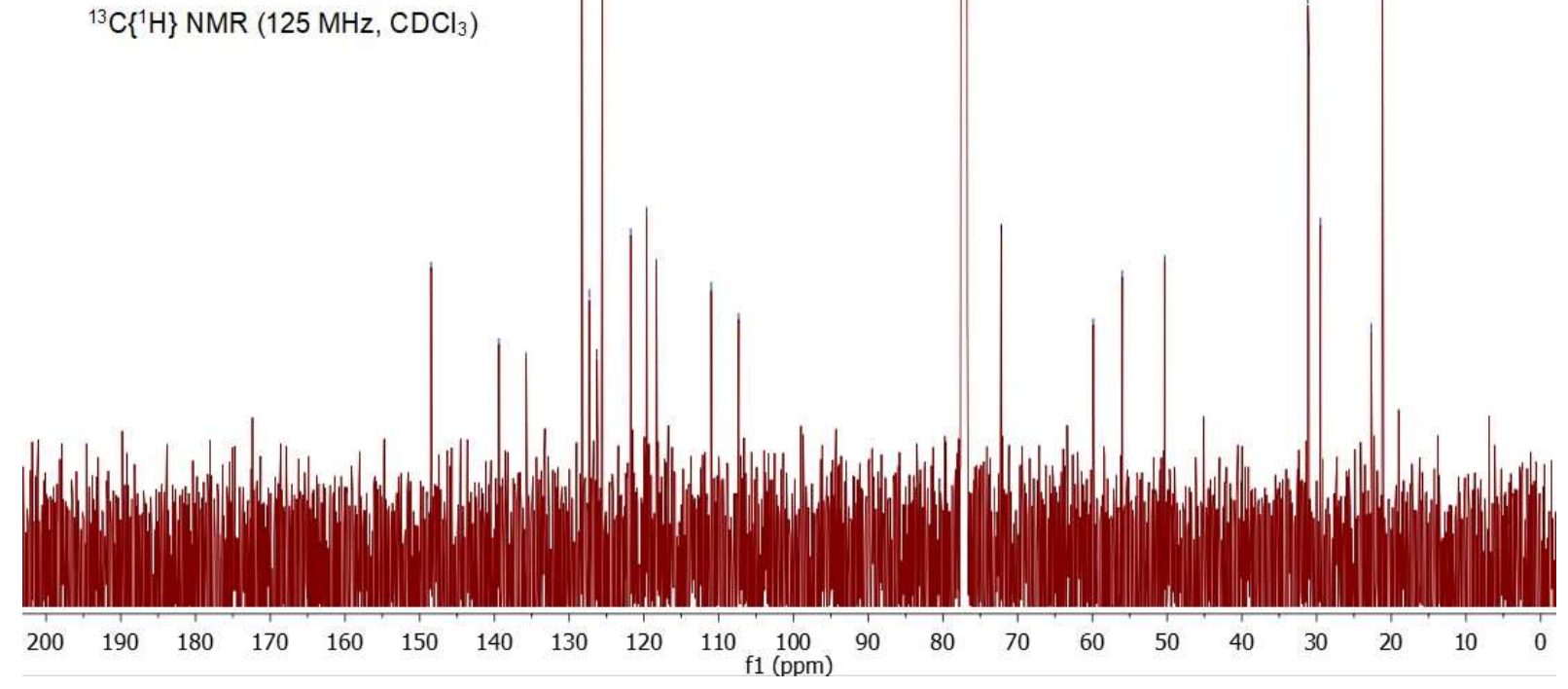

